Portland State University

PDXScholar

\title{
Crystal Growth and Surface Modification of Pyrite for Use as a Photovoltaic Material
}

Eric Rustad Young

Portland State University

Follow this and additional works at: https://pdxscholar.library.pdx.edu/open_access_etds

Part of the Chemistry Commons

Let us know how access to this document benefits you.

\section{Recommended Citation}

Young, Eric Rustad, "Crystal Growth and Surface Modification of Pyrite for Use as a Photovoltaic Material" (2018). Dissertations and Theses. Paper 4233.

https://doi.org/10.15760/etd.6117

This Dissertation is brought to you for free and open access. It has been accepted for inclusion in Dissertations and Theses by an authorized administrator of PDXScholar. Please contact us if we can make this document more accessible: pdxscholar@pdx.edu. 
Crystal Growth and Surface Modification of Pyrite for Use as a Photovoltaic Material

by

Eric Rustad Young

A dissertation submitted in partial fulfillment of the requirements for the degree of

\author{
Doctor of Philosophy \\ in \\ Chemistry
}

Dissertation Committee:

Erik Johansson, Chair

Andrea Goforth

Dean Atkinson

Erik Sanchez

Portland State University

2018 
(C) 2018 Eric Rustad Young 


\begin{abstract}
Pyrite $\left(\mathrm{FeS}_{2}\right)$ has recently attracted significant interest as a photovoltaic material due to its promising optical properties, high photon to electron conversion yield, and lowcost raw materials. However, hopes have been tempered by recent discoveries that suggest the presence of hard to remove bulk sulfur defects. This research was focused on engineering and implementing the crystallization of pyrite from a sulfur rich solution to counteract the material's natural tendency to form bulk sulfur defects. Homoeptiaxial layers and single-crystal samples have been grown from tellurium sulfur melts with an Fe:S ratio of 1:4 using both natural and synthetic substrates. The homoepitaxial layer has been characterized using scanning electron microscopy (SEM), transmission electron microscopy (TEM), and high resolution transmission electron microscopy (HRTEM), confirming the epitaxial nature of the synthetic $\mathrm{FeS}_{2}$ layer, and $\mathrm{X}$-ray and ultraviolet photoelectron spectroscopy (XPS and UPS) to better understand the energetics of the grown materials. Furthermore, epitaxial growth onto natural pyrite, in contrast to substrate etching, was established using sulfur-34 substitution and secondary ion mass spectrometry (SIMS) (Chapter 2). Growth onto synthetic pyrite was also described (Chapter 3). Finally, the photovoltaic properties of homoepitaxial layers of high temperature solution growth pyrite onto a synthetic templating crystal was characterized using electrochemical methods (Chapter 4).
\end{abstract}




\section{Dedication}

This dissertation is dedicated to my parents Annette and Stacy,

who always pushed me in the right direction until I was ready to push myself. 


\section{Acknowledgements}

The work involved in this research has been the biggest challenge of my life. I have not met it alone and I have many people to thank for helping me get to where I am. I would like to thank Dr. Erik Johansson. He welcomed me into his lab and gave me the advice, encouragement and time I needed to develop as a scientist. He was always positive and looking to the future which was much needed when I was pessimistic and stuck in the present. His strength was contagious. I am very thankful to be able to enjoy all that he generously provided.

To my committee for providing me with guidance and encouragement. To Dr. Dean Atkinson for providing insight in his electronics for chemist class. To Dr. Andrea Goforth for sending me in the right direction when I was stuck and for providing very useful feedback to make this document the best it could be. To Dr. Erik Sanchez who provided me with the materials to start my project, and if I could find him, was more than willing to help me troubleshoot any problems I had along the way.

To my lab mates and friends who I was privileged to spend time with. Dr. Qi Tong, the other member of team pyrite! She would listen to any idea I had, no matter how ridiculous it was and provide helpful feedback. To Dr. Chase Reinhart, a true friend who always encouraged my curiosity and was always there when I needed him whether it was related to this work or not. To Dr. Vitalii Dereviankin who I grew closest to during the end of this journey. Knowing that you would be there seven days a week made it easier and more enjoyable to do so myself. To my friend Capri Price who shared my work habits as unhealthy as they were and some of my recreational activities that reminded me of a world outside of this research. I wish her all the success and fulfillment she deserves. To Drs. 
Rob Jensen and Natasja Swartz who I was always happy to see and was always ready to hug. I had fun and laughed more than I thought I would and will never forget the time I spent with all of them.

If the lab was where I spent my weekdays the basement at the CEMN was where I spent my nights and weekends. For that I have Dr. Tony Chen and Greg Baty to thank. They allowed me to spend as much time as I could with their instruments. They were both always willing to help solve any problems or share helpful life experiences. Some of my most memorable times were spent in the CEMN and they would not have happened if it was not for them.

To my chemistry professors at Radford University Drs. Cindy Burkhardt, Francis Webster, and Joseph Wirgau. The three of them were able to encourage and inspire me. They planted the seeds of graduate school in my head and assure me that it was within my grasp.

To my mother Annette, father Stacy, and brother Brice. They always encouraged, supported and believed in me even when I was not able make time for them. To them I owe countless hours of phone calls and personal visits which I hope to be able to deliver. To Brian Wehner and Christie Pierce, they were both always there for me without question and I am in debt of their support.

To Katy Hosbein, she was able to give me whatever I needed, whether it was laughter, comfort or helping me to always yield to overcoming the stress of this process by relaxing. She was able to show me that life is incomplete without having cats or acting like them occasionally. 


\section{Table of Contents}

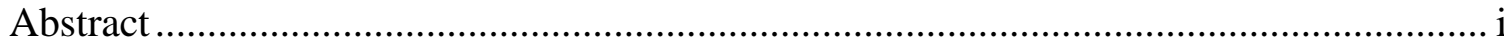

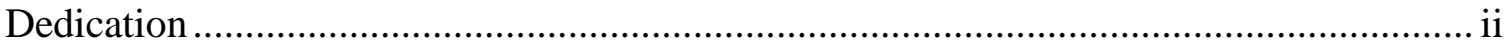

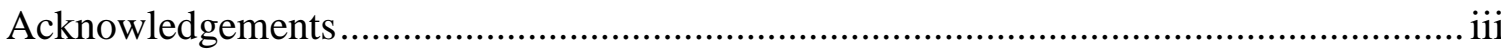

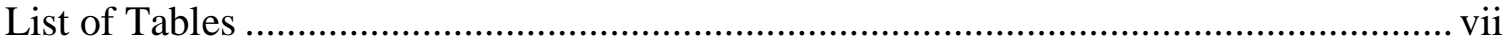

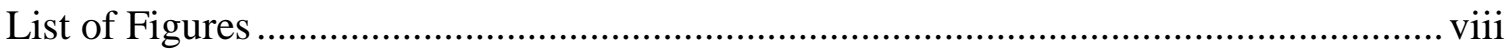

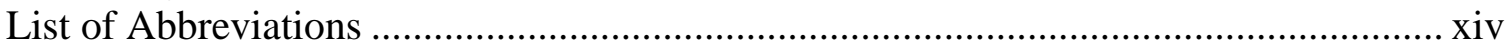

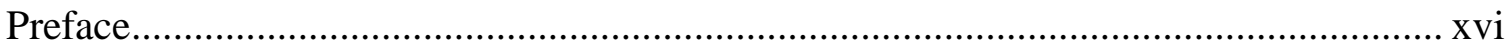

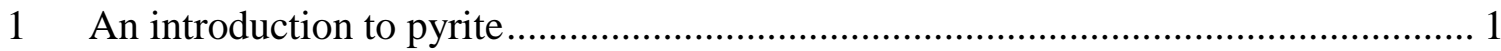

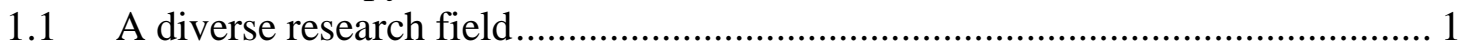

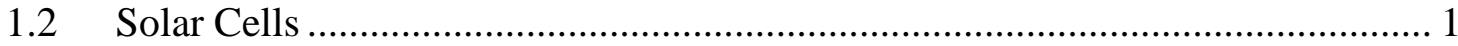

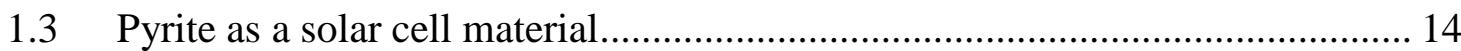

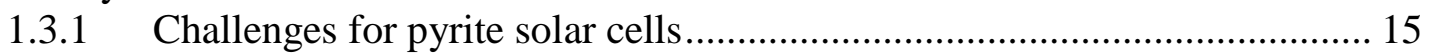

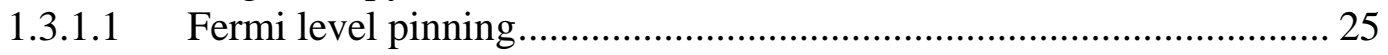

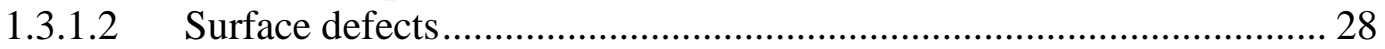

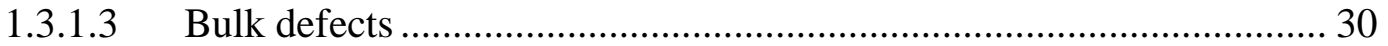

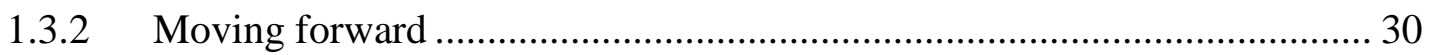

2 Crystalline growth of synthetic pyrite onto a natural substrate ……........................ 32

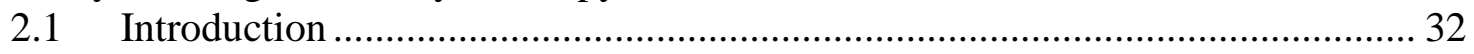

2.1.1 The challenges of pyrite synthesis ............................................................. 32

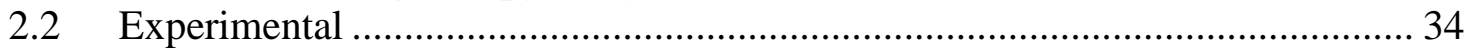

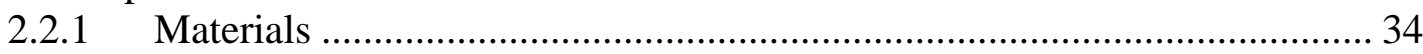

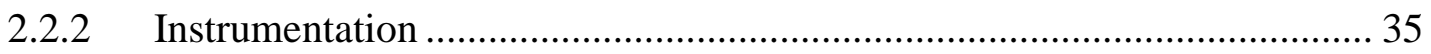

2.2.3 Method of pyrite crystallization with HTSG …………………………..... 36

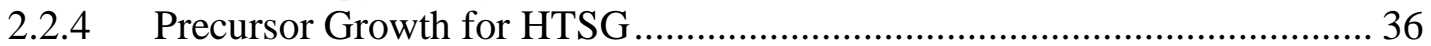

2.2.4.1 Synthesis of pyrite from Iron Pentacarbonyl ...................................... 37

2.2.4.2 Sulfur annealing iron into pyrite.......................................................... 39

2.2.5 Substrate for HTSG Preparation ……………........................................... 40

2.2.6 High Temperature Solution Growth ……………...................................... 41

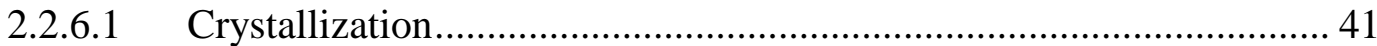

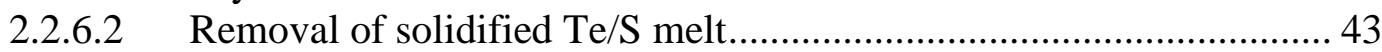

2.2.6.3 TEM preparation.......................................................................... 44

2.2.6.4 Isotope enriched growth as quantitative evidence for growth .............. 46

2.2.7 Electrochemical studies of HTSG/natural pyrite samples .......................... 49

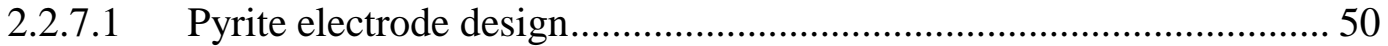

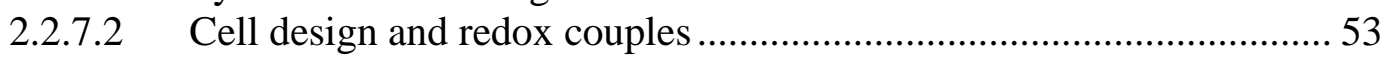

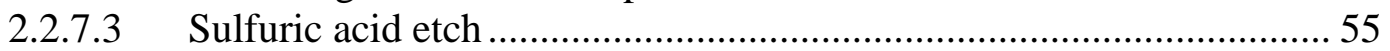

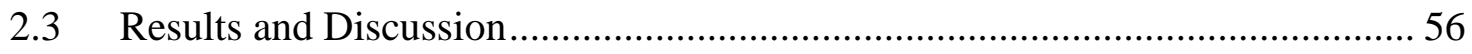

2.3.1 Precursor from iron pentacarbonyl …………….................................... 56

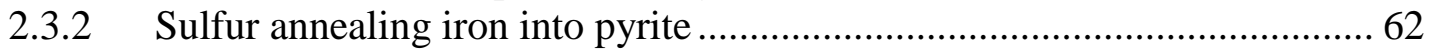

2.3.3 Qualitative evidence of HTSG onto natural pyrite ...................................... 62

2.3.4 Quantitative evidence of growth from isotope enrichment.......................... 66

2.3.5 Photovoltage measurements of HTSG onto natural pyrite .......................... 71

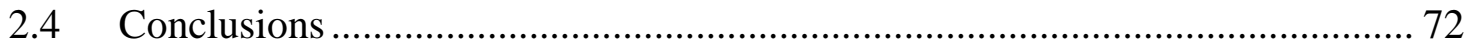


3 CVT growth of pyrite as a replacement substrate for HTSG to increase $V_{\mathrm{ph}} \ldots \ldots \ldots \ldots .73$

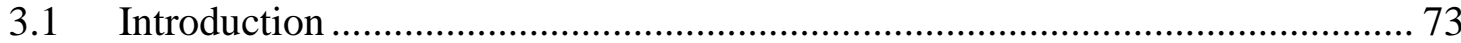

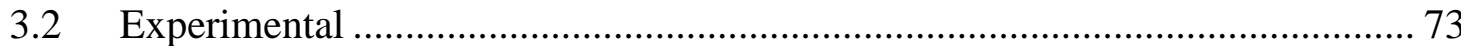

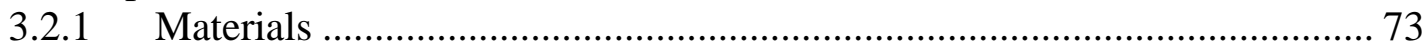

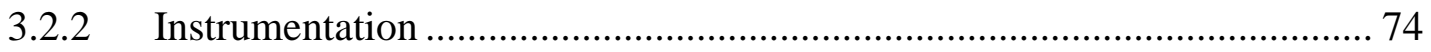

3.2.3 CVT crystal growth............................................................................... 74

3.2.4 CVT growth of an over-layer onto natural pyrite ........................................ 75

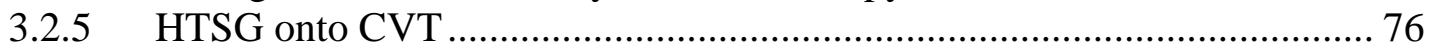

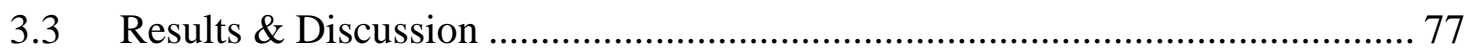

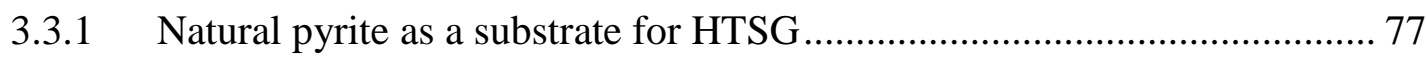

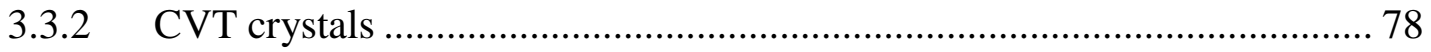

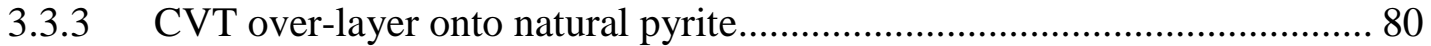

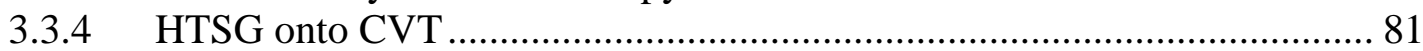

3.3.5 Electrochemical characterization of HTSG/CVT pyrite ............................. 83

3.3.6 Effects of sulfuric acid etching on the HTSG/CVT pyrite surface ............. 85

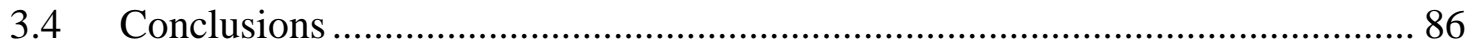

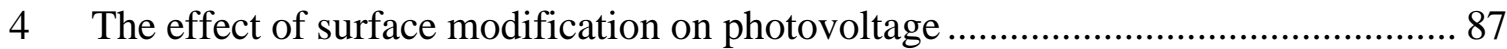

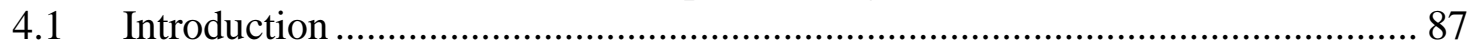

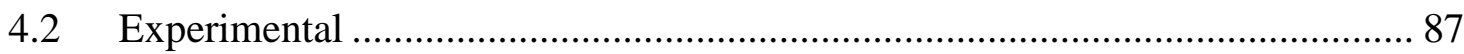

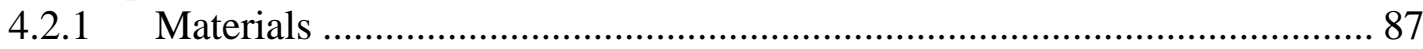

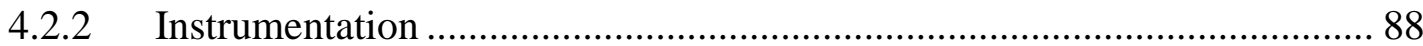

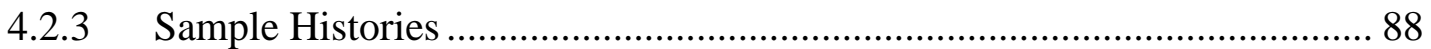

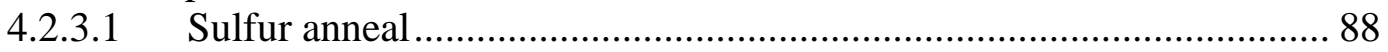

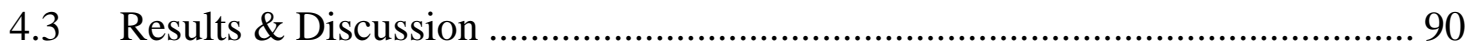

4.3.1 X-Ray photoelectron spectroscopy study of different pyrite surfaces........ 90

4.3.2 Ultraviolet photoelectron spectroscopy of different pyrite surfaces......... 101

4.3.3 $V_{\mathrm{ph}}$ measurements of four different sample histories of CVT pyrite ........ 106

4.3.4 Relationship between measured $V_{\text {ph }}$ and the surface of CVT pyrite......... 108

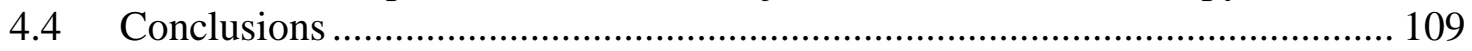

$5 \quad$ Summary and future ................................................................................... 110

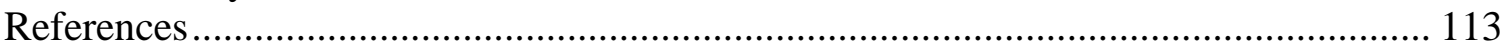

Appendix: Calculated isotope abundances ………………..................................... 123 


\section{List of Tables}

Table 2.1: Isotopic abundances of natural sulfur and iron as well as modified abundances of sulfur. $\mathrm{S}_{\mathrm{A}}$ and $\mathrm{Fe}_{\mathrm{A}}$ represent the natural abundances of the isotopes of sulfur and iron. $\mathrm{S}_{\mathrm{MA}}$ represents the modified abundances of the isotopes of sulfur due to the addition of sulfur-34.

Table 2.2. Electrochemical solutions used to characterize pyrites photovoltaic performance 55

Table 2.3: Photovoltage measurements for HTSG onto a natural pyrite crystal. ............ 71 Table 3.1. Photovoltage measurements of HTSG/natural pyrite and HTSG/CVT pyrite samples.

Table 4.1. The dielectric functions of pyrite slides before and after annealing in a sulfur atmosphere (each data was an average of five spots on the same slide) 89 Table 4.2. Photovoltage of Polished, HTSG, Etched, and sulfur annealed pyrite in 3 different electrolyte solutions at 1 sun and high intensity. 107 


\section{List of Figures}

Figure 1.1 Band formation from molecular orbitals into a crystal of pyrite. Energy is scaled so that the top is the energy of vacuum and becomes more positive down the diagram. The molecular orbitals start out from a single unit of pyrite. As more are added the two energy levels create more. In crystals this is repeated so that there is the formation of bands of continuous energy. The highest occupied molecular orbital (HOMO) is known as the valence band where the top edge in the diagram is the energy of the valence band edge $\left(\mathbf{E}_{\mathrm{vbe}}\right)$. The lowest unoccupied molecular orbital (LUMO) is known as the valence band. The bottom edge of this band is the energy of the conduction band edge $\left(\mathbf{E}_{\mathrm{cbe}}\right)$. The space between these two does not have any energy levels and is known as the band gap $\left(\mathbf{E}_{\mathrm{g}}\right)$....

Figure 1.2. Energy diagram of a $p$ - $n$ junction. (a) is at $0 \mathrm{~K}$. The bottom half of both (a) and (b) are two dimensional physical representations of the atoms in each of the materials. The black circles are the constituent atoms of the material. The red circles are donor atoms and the blue circles are acceptor atoms. $\mathbf{E}_{\mathrm{D}}$ and $\mathbf{E}_{\mathrm{A}}$ are the energy levels of the donors and acceptors. $\mathbf{E}_{\text {cbe }}$ and $\mathbf{E}_{\mathrm{vbe}}$ are the energy levels of the conduction and valence band edges. (b) is at room temperature. The atoms are labeled in the same way in (b) with the addition of free electrons as red dots, ionized constituent atoms as black circles with blue dots in them. Ionized donor atoms as blue dots with a black rim, and ionized acceptor atoms as red dots with blue rims. The Fermi level is also included on each side as a dotted line.

Figure 1.3. Energy diagram of a $p$ - $n$ junction. (a) is directly after the removal of the imaginary barrier. The black circles are the constituent atoms of the material. The red dots are free electrons. The black circles with blue dots in them are the constituent atoms that have lost an electron and now have a positive charge, this charge is known as a hole. The blue dots with thick black outlines are donor atoms that have been ionized which now have a positive charge. The blue circles with red dots in them are acceptor atoms that have accepted electrons and are now negatively charged. $\mathbf{E}_{\mathrm{D}}$ and $\mathbf{E}_{\mathrm{A}}$ are the energy levels of the donors and acceptors. $\mathbf{E}_{\mathrm{cbe}}$ and $\mathbf{E}_{\mathrm{vbe}}$ are the energy levels of the conduction and valence band edges. (b) is directly after a when no more electrons can move from the n-type to the p-type. The Fermi level is also included on each side as a horizontal dotted line. The bottom half of both (a) and (b) are two dimensional physical representations of the atoms in each of the materials. In (b) the width $W$ is the depletion width and the widths $W_{\mathrm{D}}$ and $W_{\mathrm{A}}$ are the depletion widths on the donor and acceptor doped semiconductors.

Figure 1.4 (a) is the concentration of donors at the interface of a $p$ - $n$ junction where the ntype is at positive distance and p-type at a negative, (b) the electric field built up at the same location, (c) the potential built up at the same location, (d) the band bending at the same location. (e) is the energy band diagram resulting from band bending caused by the electric field. The black circles are the constituent atoms of the material. The red dots are free electrons. The black circles with blue dots in them are the constituent atoms that 
have lost an electron and now have a positive charge, this charge is known as a hole. The blue dots with thick black outlines are donor atoms that have been ionized which now have a positive charge. The blue circles with red dots in them are acceptor atoms that have accepted electrons and are now negatively charged. $\mathbf{E}_{\mathrm{D}}, \mathbf{E}_{\mathrm{A}}, \mathbf{E}_{\mathrm{cbe}}, \mathbf{E}_{\mathrm{vbe}}$ and $\mathbf{E}_{\mathrm{F}}$ are the energy levels of the donors, acceptors, conduction band edge, valence band edge and Fermi level. The bottom half of (e) is a two-dimensional physical representation of the atoms in each of the junction. $W$ is the depletion width and the widths $W_{\mathrm{D}}$ and $W_{\mathrm{A}}$ are the depletion widths on the donor and acceptor doped semiconductors 10

Figure 1.5. $(\mathrm{a}, \mathrm{b})$ energy band diagram of $p$ - $n$ junction. The black circles are the constituent atoms, the red dots are free electrons, the black circles with blue dots in them are constituent atoms that have lost an electron and now have a positive charge, and this charge is known as a hole. The blue dots with thick black outlines are donor atoms that have been ionized which now have a positive charge. The blue circles with red dots in them are acceptor atoms that have accepted electrons and are now negatively charged. $\mathbf{E}_{\mathrm{D}}, \mathbf{E}_{\mathrm{A}}, \mathbf{E}_{\mathrm{cbe}}, \mathbf{E}_{\mathrm{vbe}}$ and $\mathbf{E}_{\mathrm{F}}$ are the energy levels of the donors, acceptors, conduction band edge, valence band edge and Fermi level. The bottom half of $(a, b)$ is a two dimensional physical representations of the atoms in each of the junction. $W$ is the depletion width and the widths $W_{\mathrm{D}}$ and $W_{\mathrm{A}}$ are the depletion widths on the donor and acceptor doped semiconductors. (a) is at the introduction of the first photon with energy equal to the band gap. This photon labeled $h v$ is introduced into the junction which is labeled as arrow 1. The photon is absorbed by an electron and promoted to the conduction band this is labeled as arrow 2. The electron is then carried by the drift of the electric field into the ntype side of the semiconductor labeled arrow 3. The hole created in the conduction band by step 2 is then combined with an electron from the valence band from the p-type side labeled arrow 4. This is also shown in (b) which is after the net movement of electrons is zero. (b) does not have a fermi level but two quasi fermi levels labeled $\mathbf{E}_{\mathrm{Fn}}$ and $\mathbf{E}_{\mathrm{Fp}} \ldots . . .12$ Figure 1.6. Current-voltage (I- $V$ ) characteristics of a typical photovoltaic cell with a good fill factor $(F F)$ (a) and typical I- $V$ curve for a $\mathrm{FeS}_{2} \mathrm{I}^{-} / \mathrm{I}_{3}{ }^{-}$cell (b) with lower $F F$ values. Box A has the height of the short circuit current density $\left(j_{\mathrm{sc}}\right)$ and the width of the photovoltage $\left(V_{\mathrm{ph}}\right)$. At each point of the plot the product of the current and voltage is equal to the power that can be produced. Box B's height and width are determined from the max power point. In (a) box B corresponds to a $F F$ of 0.8 . In (b) box B corresponds to a $F F$ of 0.5 . In (b) box $C$ has a fill fact of 0.8 .

Figure 1.7 Energy diagram of an n-type semiconductor in an electrolyte solution (a) at 0 $\mathrm{K}$ and (b) at room temperature. The bottom half of both (a) and (b) are two dimensional physical representations of the atoms in each of the materials. The black circles are the constituent atoms of the material. The red circles are donor atoms. $\mathbf{E}_{\mathrm{D}}, \mathbf{E}_{\mathrm{cbe}}, \mathbf{E}_{\mathrm{vbe}}, \mathbf{E}_{\mathrm{F}}$, and $E\left(\mathrm{~A} / \mathrm{A}^{-}\right)$are the energy levels of the donors, conduction and valence band edges, the Fermi level, and the solution potential. The atoms are labeled in the same way in b with the addition of free electrons as red dots, ionized constituent atoms as black circles with blue dots in them. Ionized donor atoms as blue dots with a black rim. 
Figure 1.8 Energy diagram of an n-type semiconductor in an electrolyte solution. (a) is right after the barrier between the two is removed. The black circles are the constituent atoms of the material. The red dots are free electrons. The black circles with blue dots in them are ionized constituent atoms. The blue dots with thick black rims are ionized donor atoms. $\mathbf{E}_{\mathrm{D}}, \mathbf{E}_{\mathrm{cbe}}, \mathbf{E}_{\mathrm{vbe}}, \mathbf{E}_{\mathrm{F}}$, and $E\left(\mathrm{~A} / \mathrm{A}^{-}\right)$are the energy levels of the donors, conduction and valence band edges, the Fermi level, and the solution potential. The atoms are labeled in the same way in (b) (b) is at equilibrium when the electrons from the n-type semiconductor are no longer able to move into the solution due to the buildup of the electric field at the junction. The bottom half of both (a) and (b) are two dimensional physical representations of the atoms in each of the materials.

Figure 1.9 Energy diagram of an n-type semiconductor in an electrolyte solution. The black circles are the constituent atoms of the material. The red dots are free electrons. The black circles with blue dots in them are ionized constituent atoms. The blue dots with thick black rims are ionized donor atoms. $\mathbf{E}_{\mathrm{D}}, \mathbf{E}_{\mathrm{cbe}}, \mathbf{E}_{\mathrm{vbe}}, \mathbf{E}_{\mathrm{F}}, E\left(\mathrm{~A} / \mathrm{A}^{-}\right), \mathbf{E}_{\mathrm{Fn}}$ and $\mathbf{E}_{\mathrm{Fp}}$ are the energy levels of the donors, conduction and valence band edges, the Fermi level, the solution potential, the quasi Fermi level of electrons, and the quasi Fermi level of holes. (a) is at the introduction of the first photon with energy equal to the band gap. This photon labeled $h v$ is introduced into the junction which is labeled as 1 . The photon is absorbed by an electron and promoted to the conduction band this is labeled as 2 . The electron is then carried by the drift of the electric field into the n-type side of the semiconductor labeled 3. The hole created in the conduction band by step 2 is then combined with an electron from the valence band from the p-type side labeled 4 . This is also shown in (b) which is after the net movement of electrons is zero.

Figure 1.10 Formation of depletion region due to metallic band surface states. (a) at $0 \mathrm{~K}$, (b) after equilibrium, (c) depletion region formation. Red circles are dopant atoms, blue circles are acceptors. Red dots are free electrons, blue dots with black rims are ionized donor atoms, red dot with blue rims are ionized acceptor atoms. $\mathbf{E}_{\mathrm{cbe}}, \mathbf{E}_{\mathrm{D}}, \mathbf{E}_{\mathrm{F}}, \mathbf{E}_{\mathrm{vbe}}, \mathbf{E}_{0}$ are the energy levels of the conduction band edge, donor atoms, fermi level, valence band edge and charge neutrality.

Figure 1.11 Energy diagram of junction of n-type material and its surface layer. (a) at equilibrium, (b) first incident photon, (c) at the limit of charge separation. Red circles are dopant atoms, blue circles are acceptors. Red dots are free electrons, blue dots with black rims are ionized donor atoms, red dot with blue rims are ionized acceptor atoms. $\mathbf{E}_{\mathrm{cbe}}, \mathbf{E}_{\mathrm{D}}$, $\mathbf{E}_{\mathrm{F}}, \mathbf{E}_{\mathrm{vbe}}, \mathbf{E}_{0}$ are the energy levels of the conduction band edge, donor atoms, fermi level, valence band edge and charge neutrality. (b) is at the introduction of the first photon with energy equal to the band gap. This photon labeled $h v$ is introduced into the junction which is labeled as 1 . The photon is absorbed by an electron and promoted to the conduction band this is labeled as 2 . The electron is then carried by the drift of the electric field into the n-type side of the semiconductor labeled 3. The hole created in the conduction band by step 2 is then combined with an electron from the valence band from 
the p-type side labeled 4. This is also shown in (c) which is after the net movement of electrons is zero.

Figure 2.1: Substrate preparation. First the cube is polished on all six sides. Then one of the sides is cut producing a slide of $2 \mathrm{~mm}$ thickness. Then the backside of the slide is polished.

Figure 2.2: Homoeptiaxial layer growth: (a) assembling of the ampoule, tellurium, synthetic pyrite powder, sulfur and a prepared substrate are loaded into a quartz ampoule. The ampoule is then evacuated to $5 \times 10^{-7}$ torr and sealed with a torch. (b) the ampoule is heated to $650{ }^{\circ} \mathrm{C}$ where it remains for 12 hours. (c) the ampoule is then inverted so that the melt is covering the substrate and is then cooled to $550{ }^{\circ} \mathrm{C}$ at a rate of $1 / 3{ }^{\circ} \mathrm{C} / \mathrm{h}$. (e) the ampoule is inverted again to remove the melt from the substrate.

Figure 2.3: Diagram for TEM sample preparation. Brown indicates the substrate (natural pyrite) and orange indicates the synthetic growth layer. First the epilayer is grown onto the natural substrate. Then FIB is used to mill out the area on the edge of one of the growths. Then the lamella is attached to a probe and removed from the sample.

Figure 2.4: Diagram of SIMS experimental design with predicted depth profile outcome. In the diagram brown represents the natural pyrite substrate and orange represents the synthetic epilayer. HTSG is carried out onto a natural slide of pyrite. Then a depth profile is created by milling into the surface and mass analyzing the removed material. Based on the level of doping there should are two predicted ratios of pyrite, one made of natural levels of sulfur and one of pyrite with doped levels of sulfur-34. 47

Figure 2.5. Schematic of classic style of pyrite electrode......................................... 51

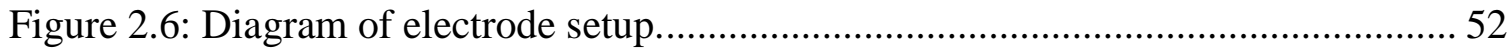

Figure 2.7: Electrochemical cell schematic. ......................................................... 54

Figure 2.8: XRD pattern of synthetic pyrite directly after solvent removal (red) and after sulfur annealing (blue). References for pyrite (black) and marcasite (green) are included in the figure. 57

Figure 2.9: Precursor SEM.: SEM images of $\mathrm{FeS}_{\mathrm{x}}$ powder before $(\mathrm{a}, \mathrm{b}, \mathrm{c})$ and after $(\mathrm{d}, \mathrm{e}, \mathrm{f})$ sulfur annealing. An image was taken for each at relatively the same magnification for visual comparison.

Figure 2.10. Raman of synthetic pyrite before (a) and after (b) sulfur annealing. Pyrite (blue) and marcasite (red) references are included in each......................................... 61 Figure 2.11. Raman spectrum of twice sulfur annealed Fe. A pyrite reference (blue) is included.

Figure 2.12: SEM images of natural slide having undergone HTSG with the solidified melt removed.

Figure 2.13: TEM sample preparation and a micrograph of the growth mesa. Orange is the synthetic epilayer and brown is the underlying natural substrate. 65 Figure 2.14: HRTEM with orientation identification. In the diagram to the left the orange is the synthetic epilayer and the brown is the underlying natural substrate. 66 
Figure 2.15: Control SIMS depth profile on a polished natural pyrite slide. The orange line is the predicted ratio of isotopes for the synthetic layer. The brown is for the natural layer. b) is a plot of the depth measured by profilometry vs the sputter time. 67

Figure 2.16: SEM image of the area where the SIMS was performed. ............................ 68 Figure 2.17: a) diagram of the profiling process, here the brown represents the natural substrate and orange represents the synthetic epilayer. b) the area that was profiled with four different regions of interest selected. c) the corresponding depth profiles of each of the four spots including predicted ratios in orange for natural and brown for synthetic. . 69 Figure 2.18. Sputtering of two different surfaces. One with a flat layer grown on the surface to the left. On the right an uneven surface growth. Dotted lines represent new surface boundaries after material removal................................................................... 70 Figure 3.1. Schematic for CVT growth onto natural pyrite and then remove of natural pyrite to leave only the CVT pyrite for use in the HTSG. 76 Figure 3.2. Above, a schematic of the homoepitaxial CVT growth. The hot zone is at 650 ${ }^{\circ} \mathrm{C}$ for 12 days. The source is transported to the seed crystal by the addition of $\mathrm{FeBr}_{2}$. Below is a photograph of the ampoule after growth.................................................... 76 Figure 3.3: SEM of defects in natural pyrite post HTSG. ............................................ 77 Figure 3.4: XRD pattern of ground up CVT pyrite (purple) with a pyrite reference included (black). ..................................................................................................... 78 Figure 3.5. Raman spectroscopy of CVT pyrite. A pyrite reference (blue) is included... 79 Figure 3.6: CVT pyrite grown onto polished natural pyrite. top is from one growth,

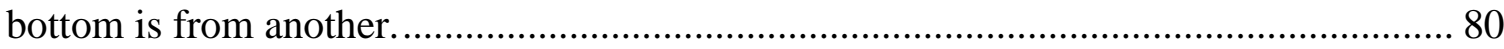
Figure 3.7: HTSG onto a CVT crystal. a-d) are all the surface of the substrate................ 81 Figure 3.8. Raman of HTSG/CVT. A pyrite reference (blue) is included......................... 82 Figure 3.9: I- $V$ curve for etched HTSG/CVT pyrite contacted with a) $\mathrm{Cp}_{2} \mathrm{Fe}_{2} / \mathrm{Cp}_{2} \mathrm{Fe}^{+}$, and

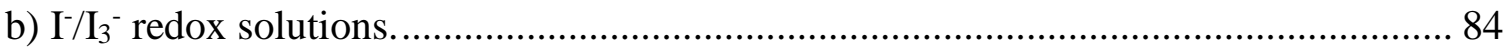
Figure 3.10: Surface HTSG CVT pyrite after electrochemical etching............................ 85 Figure 4.1. EBSP (Kikuchi patterns) of a pyrite slide (a) pre- and (b) post-sulfur anneal. (c) and (d) are auto index results of two different spots on the annealed slide. 90 Figure 4.2: XPS survey scan of polished, sulfur annealed, etched and HTSG CVT pyrite.

Figure 4.3: Normalized and background subtracted XPS spectra of the Fe2p3/2 range of polished, HTSG, etched in $\mathrm{H}_{2} \mathrm{SO}_{4}$ with a cathodic potential applied and sulfur annealed, CVT pyrite. 94

Figure 4.4. Normalized and background subtracted XPS spectra of the S2p range of polished, HTSG, etched in $\mathrm{H}_{2} \mathrm{SO}_{4}$ with a cathodic potential applied and sulfur annealed, CVT pyrite. 99

Figure 4.5: Normalized UPS spectra of CVT pyrites with 4 different sample histories, polished, through HTSG, through a cathodic etch in $0.5 \mathrm{M} \mathrm{H}_{2} \mathrm{SO}_{4}$ and sulfur annealed at $650{ }^{\circ} \mathrm{C}$ for 12 hours 102 
Figure 4.6. UPS spectra of the outer valence band of the CVT pyrite through different histories, polished, HTSG, etched, and sulfur annealed. The spectra have been normalized

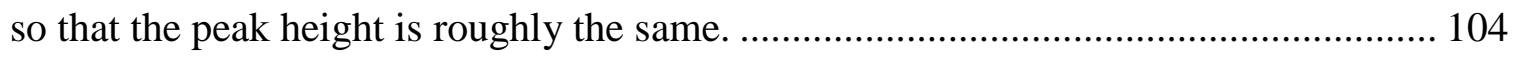
Figure 4.7. Band diagram for CVT samples with different histories generated from UPS spectra and assuming an electronic bandgap of $0.8 \mathrm{eV}$. The reduction potentials of the electrolyte solutions are included as well. 105 


\section{List of Abbreviations}

CVT - chemical vapor transport

DFT: density functional theory

DFT-GGA: density functional theory generalized gradient approximations

$D_{\text {it }}$ - density of interface states

$E$ - potential

$E^{\circ}$ - standard potential

$\mathbf{E}_{0}$ - charge neutral energy level

$\mathbf{E}_{\mathrm{A}}-$ energy level of acceptors

$\mathbf{E}_{\text {cbe }}$ - conduction band edge energy level

$\mathbf{E}_{\mathrm{D}}$ - energy level of donors

EDX: energy dispersive spectroscopy

$\mathbf{E}_{\mathrm{F}}-$ Fermi level energy

$\mathbf{E}_{\mathrm{g}}$ - bandgap energy

$\mathbf{E}_{\mathrm{vac}}$ - energy of vacuum

$\mathbf{E}_{\mathrm{vbe}}$ - valence band edge energy level

$F F$ - fill factor

HOMO - highest occupied molecular orbital

HRTEM - high resolution transmission electron spectroscopy

HTSG - high temperature solution growth

$h v$ - planks constant, frequency, energy of light

ICP-MS - inductively coupled plasma mass spectrometry

$I_{\mathrm{sc}}-$ short circuit current

ISW - iron sulfur world

$J_{\mathrm{sc}}-$ short circuit current density

$k$ - Boltzmann constant

LUMO - lowest unoccupied molecular orbital

MPP - maximum power point

$N_{\mathrm{A}}-$ acceptor density

$N_{\text {D }}$ - donor density

$n_{e}-$ stoichiometric number of electrons involved in redox reaction

$\mathrm{PCE}$ - photon to electron conversion efficiency

$P_{\text {in: }}$ power in

$q$ - elementary charge

$Q_{\text {sc }}$ - charge on semiconductor

$Q_{\text {ss }}$ - charge of surface states

$R_{\text {ser }}-$ series resistance

$R_{\text {sh }}-$ shunt resistance

SEM - scanning electron microscopy

SIMS: secondary ion mass spectrometry

STM - scanning tunneling microscopy

$T$ - temperature

TEM - transmission electron microscopy

ToF-SIMS: time of flight secondary ion mass spectrometry 
UPS - ultraviolet photoelectron spectroscopy

$V_{\text {ph }}$ - photovoltage

$W$ - depletion width

$W_{\text {A }}$ - depletion width on semiconductor doped with acceptors

$W_{\mathrm{D}}$ - depletion width on semiconductor doped with donors

XPS - x-rap photoelectron spectroscopy

XRD - X-ray diffraction

$\Psi_{\text {bi }}$ - built-in voltage

$\varepsilon_{\mathrm{A}}$ - electric field on semiconductor doped with acceptors

$\varepsilon_{\mathrm{D}}$ - electric field on semiconductor doped with donors

$\varepsilon_{\mathrm{S}}$ - static dielectric constant of semiconductor 


\section{Preface}

This thesis is based on the work contained in the following papers:

I Tong, Q.; Young, E.R.; Johansson, E. Infrared Reflection-Absorption Spectroscopy of an Isotropic Adsorbate layer on Pyrite J. Phys. Chem. C. 2013, 117, 25982-25990 II (manuscript in preparation)

III (manuscript in preparation)

The contribution of the author to the papers included in this thesis was as follows:

I. Participated in planning the study. Performed some of the laboratory work. Second writer of manuscript.

II. Participated in planning the study. Performed all the laboratory work. Main writer of manuscript.

III. Participated in planning the study. Performed all the laboratory work. Main writer of the manuscript. 


\section{An introduction to pyrite}

\subsection{A diverse research field}

Pyrite $\left(\mathrm{FeS}_{2}\right)$ has received a lot of attention in many different research areas. $\mathrm{FeS}_{2}$ has attracted considerable attention over the past decades as an energy-conversion material, as a common by-product of mining, and as a factor in some origin-of-life theories. As a matter of fact, $\mathrm{FeS}_{2}$ has mainly been studied for purposes other than those related to solarenergy conversion. Pyrite is commonly encountered during mining operations and it is found in both gold and coal among many other materials; thus, effectively removing pyrite is crucial to recovering the desired compounds. ${ }^{1}$ Furthermore, oxidative decomposition of pyrite in the presence of $\mathrm{O}_{2}$ and $\mathrm{H}^{+}$leads to acid mine drainage and production of $\mathrm{H}_{2} \mathrm{~S}$, a toxic gas. ${ }^{2,3}$ In the origin-of-life field the "iron-sulfur world" (ISW) has attracted much interest and it has been theorized that pre-biotic chemistry took place under extreme conditions at the interface between water and Fe/S minerals. ${ }^{4}$ However, the topic of this work is pyrite as a solar cell material. Prior to describing pyrite, the details of solar cells will be discussed.

\subsection{Solar Cells}

The purpose of a solar cell is to convert the energy of light into electrical energy. This is most commonly done with semiconductors being the active light absorbing materials. Semiconductors are unique in that they have a conductive character that is in between conductors and insulators. Their electrical properties can be manipulated by doping. Doping is the substitution of constituent atoms with different atoms. Many semiconductors are crystals that are made of a single building block that is repeated in all 
three physical dimensions. The periodicity results in the molecular orbitals of the single units combining and producing more orbitals. Each combination of atomic orbitals produces one of higher and one of a lower energy molecular orbital. Repeating this with many more atomic and molecular orbitals results in a large grand total of molecular orbitals as one crystal. Based on the lattice of pyrite, the number of $\mathrm{Fe}$ atoms in a single of the repeating units, the molecular weight, and Avogadro's constant pyrite's molecular density is calculated to be $2.5 \times 10^{22}$ molecules $/ \mathrm{cm}^{3}$. This results in many energy levels close enough together that they form bands which represent a continuum of energy levels as opposed to their original discrete energy levels. This idea is depicted in Figure 1.1. 


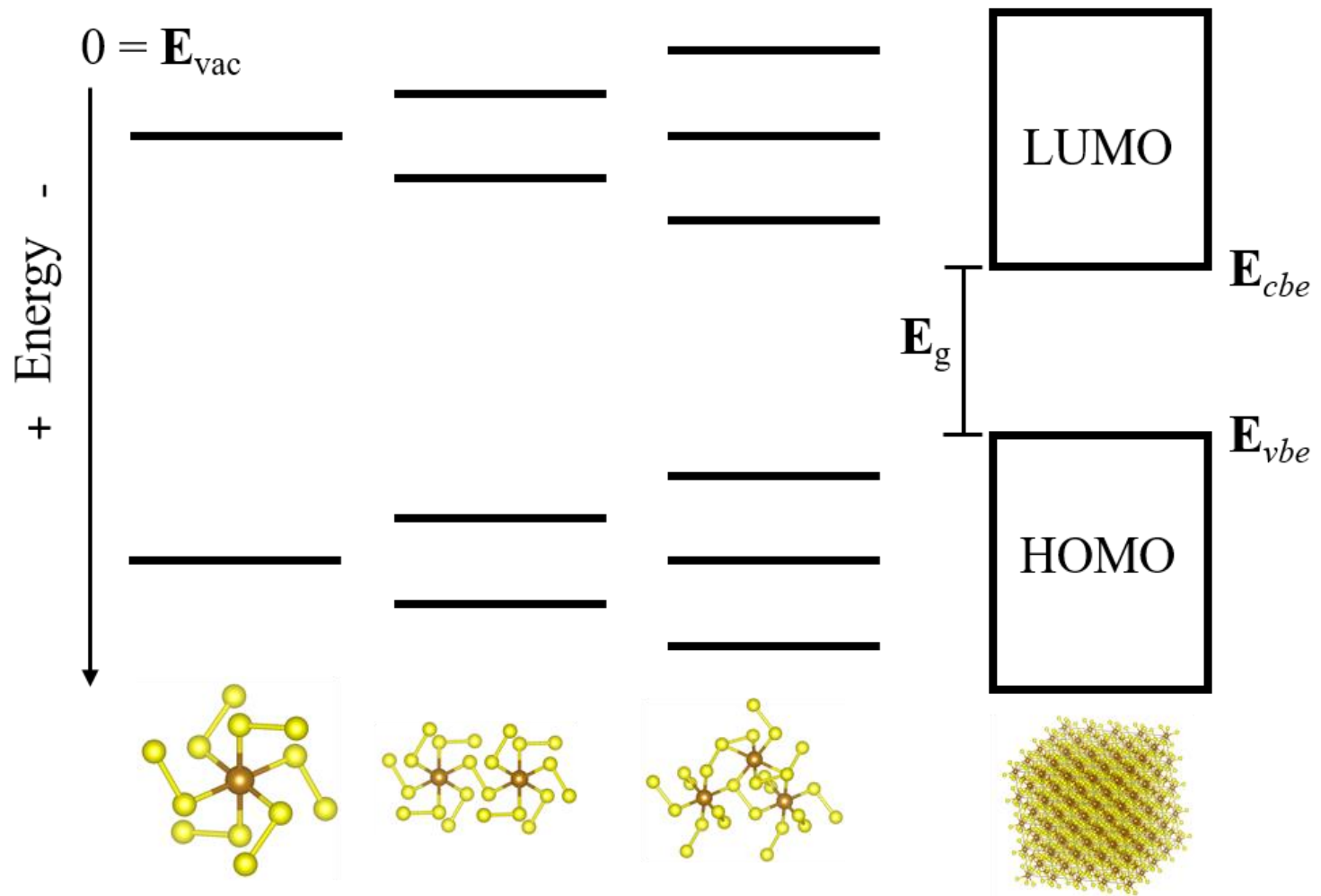

Figure 1.1 Band formation from molecular orbitals into a crystal of pyrite. Energy is scaled so that the top is the energy of vacuum and becomes more positive down the diagram. The molecular orbitals start out from a single unit of pyrite. As more are added the two energy levels create more. In crystals this is repeated so that there is the formation of bands of continuous energy. The highest occupied molecular orbital (HOMO) is known as the valence band where the top edge in the diagram is the energy of the valence band edge ( $\left.\mathbf{E}_{\mathrm{vbe}}\right)$. The lowest unoccupied molecular orbital (LUMO) is known as the conduction band. The bottom edge of this band is the energy of the conduction band edge $\left(\mathbf{E}_{\text {cbe }}\right)$. The space between these two does not have any energy levels and is known as the band gap $\left(\mathbf{E}_{\mathrm{g}}\right)$.

In Figure 1.1 the left starts with the molecular orbitals of a single pyrite molecule. Moving towards the right is the accumulation of more pyrite molecules into the bands of the crystal. The lower band is the highest occupied molecular orbital and the one above it is the lowest unoccupied molecular orbital. These are referred to as the valence band and the conduction band. $\mathbf{E}_{\mathrm{cbe}}$ is the energy of the conduction band edge, the bottom level of conduction band, and $\mathbf{E}_{\mathrm{vbe}}$ is the energy of the valence band edge, the highest level in the band. The difference between these two levels is the band gap whose energy is represented 
by the term $\mathbf{E}_{\mathrm{g}}$. This gap is unique to semiconductors. For conductors these two bands overlap and for insulators the gap is much larger, and the energy required to promote an electron to the LUMO is enough to break the bonds of the molecule. Below the diagrams of the bands the crystal structure for pyrite is shown according to the number of orbitals shown in the diagram. The energy levels in the diagram are referenced to vacuum as zero energy. This is the convention that electrochemist use which is based on work function, physicist use a more negative value for more tightly bound electrons.

At room temperature there is enough energy to promote an electron from the valence band to the conduction band. This leaves an electron vacancy in the valence band, which has a positive charge and is commonly known as a "hole". The amount that this occurs varies in semiconductors and is dependent on the size of the bandgap and the temperature. The density of electrons in the valence band and holes in the conduction band is known as the intrinsic carrier concentration and can range from $10^{6}$ to $10^{12} \mathrm{~cm}^{-3}$ which is very small compared to the density of molecules of a semiconductor like pyrite $\left(10^{22} \mathrm{~cm}^{3}\right)$.

An important energy level of the semiconductor band diagram is the Fermi level. This is the electrochemical potential of the electron in the semiconductor, this is the amount of energy required to add an electron. It is a thermodynamic quantity and represents the level with a 50\% probability of occupation. The Fermi level does not have to be at an actual energy level, it does not for intrinsic (undoped) semiconductors instead it is in the bandgap. The semiconductor can be doped to create levels within the bandgap to alter the Fermi level energy. The semiconductor that is doped is known as an extrinsic semiconductor. A solar 
cell is often the result of the junction of the same semiconductor material that has been doped in two different ways. At the junction, electrons flow to the material of the more positive Fermi level.

The junction formed is known as a $p-n$ junction. $\mathrm{P}$ and $\mathrm{n}$ refer to the doping types. P-type doping involves substituting an atom of the semiconductor with an element that has fewer valence electrons. Ideally this creates an electronic level in the band gap above the valence band edge. This energy level is close enough to the valence band that an electron can leave and occupy the energy level. This is a shallow acceptor level, the further away from the valence band it is the deeper it is. This energy level accepts the electron and gains a negative charge, which is localized on the dopant atom. The electron vacancy in the valence band is known as a hole, which has a positive charge and is on a constituent atom of the semiconductor. P-type doping adds more holes to the material without adding electrons making holes the majority carrier. This type of doping brings the Fermi level closer to the valence band making it more positive.

N-type doping involves substituting an atom of the semiconductor with an atom of an element with a greater number of valence electrons. This produces an electronic level below the conduction band edge that is occupied with an electron. This is a shallow donor, the further away from the conduction band the energy level is the deeper it is. Ideally the level is shallow enough that the electron can be donated into the conduction band at room temperature. This gives a negative charge to the conduction band and leaves a positive charge at the energy level on the dopant atom. This type of doping adds electrons to the 
semiconductor without adding holes. Electrons are now the majority carrier and the Fermi level is closer to the conduction band.

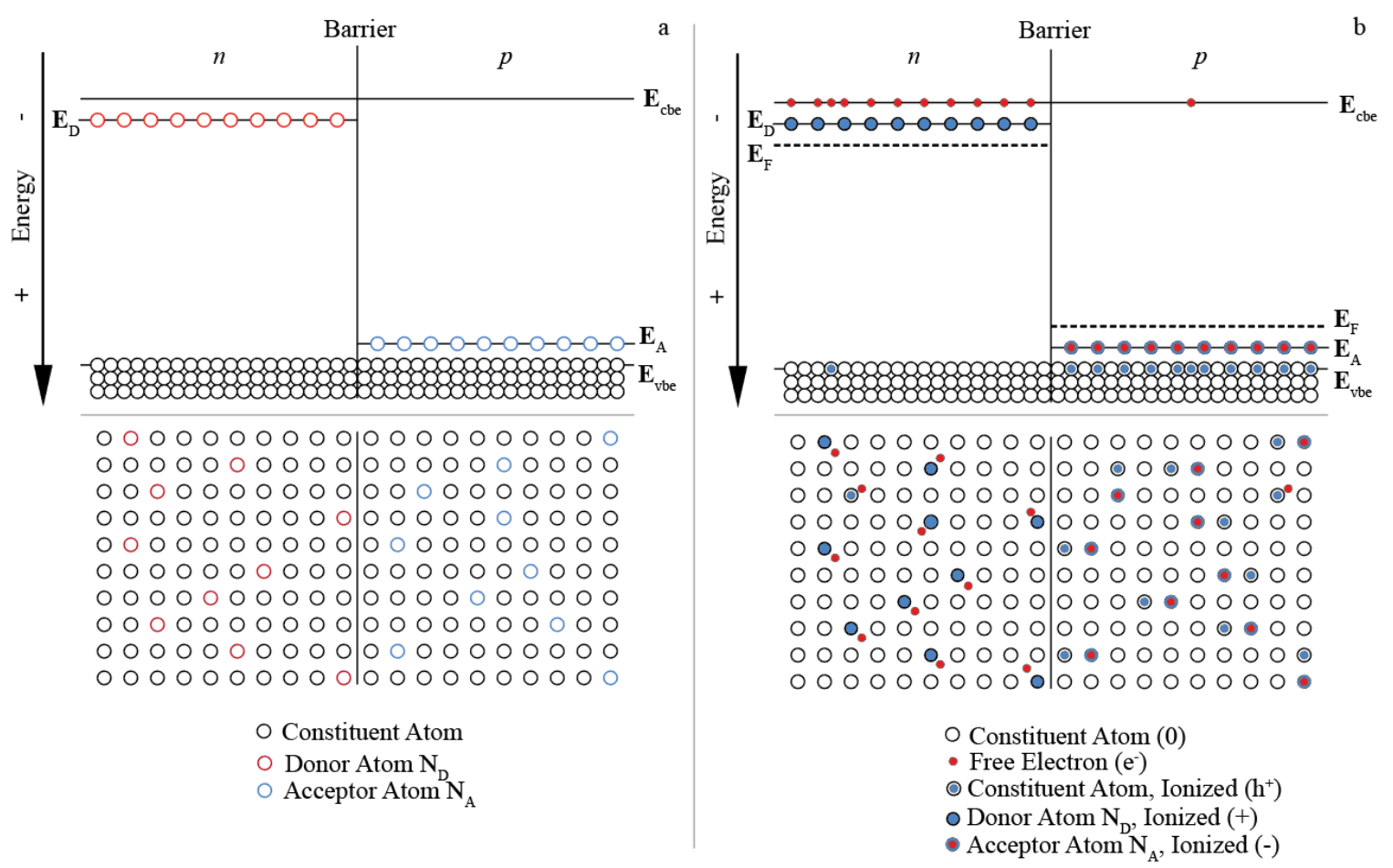

Figure 1.2. Energy diagram of a $p-n$ junction. (a) is at $0 \mathrm{~K}$. The bottom half of both (a) and (b) are two dimensional physical representations of the atoms in each of the materials. The black circles are the constituent atoms of the material. The red circles are donor atoms and the blue circles are acceptor atoms. $\mathbf{E}_{\mathrm{D}}$ and $\mathbf{E}_{\mathrm{A}}$ are the energy levels of the donors and acceptors. $\mathbf{E}_{\mathrm{cbe}}$ and $\mathbf{E}_{\mathrm{vbe}}$ are the energy levels of the conduction and valence band edges. (b) is at room temperature. The atoms are labeled in the same way in (b) with the addition of free electrons as red dots, ionized constituent atoms as black circles with blue dots in them. Ionized donor atoms as blue dots with a black rim, and ionized acceptor atoms as red dots with blue rims. The Fermi level is also included on each side as a dotted line.

Figure 1.2.a shows the junction of a $\mathrm{n}$ and p-type material at $0 \mathrm{~K}$ so there is not enough energy to promote electrons into the conduction band in the n-type material or to promote electrons into the acceptor level or the conduction band in the p-type material. There is an imaginary barrier between the two so that there is no electronic interaction between the two of them. The top of the figure shows an energy diagram like the one 
described before. There is an energy for the conduction band edge as well as the valence band edge. The black circles at and below the conduction band edge represent the constituent atoms of the semiconductor. The n-type material on the left has an energy level of the donor atoms, $\mathbf{E}_{\mathrm{D}}$. These levels are represented by red circles. The p-type material on the right has an energy level, $\mathbf{E}_{\mathrm{A}}$, from the acceptor atoms as blue circles. Below the diagram is a physical representation of the semiconductor material. This also has the constituent atoms and donor atoms labeled.

Raising the temperature results in Figure 1.2.b. Electrons from dopant atoms and much fewer constituent atoms have been donated to the conduction band of the n-type semiconductor and electrons from the constituent atoms have been accepted to the dopant levels of the p-type semiconductor and much fewer electrons have been promoted from the valence band to the conduction band. It is now clear that the n-type material's free electrons greatly outnumber its holes and the p-type materials holes greatly outnumber its free electrons. 

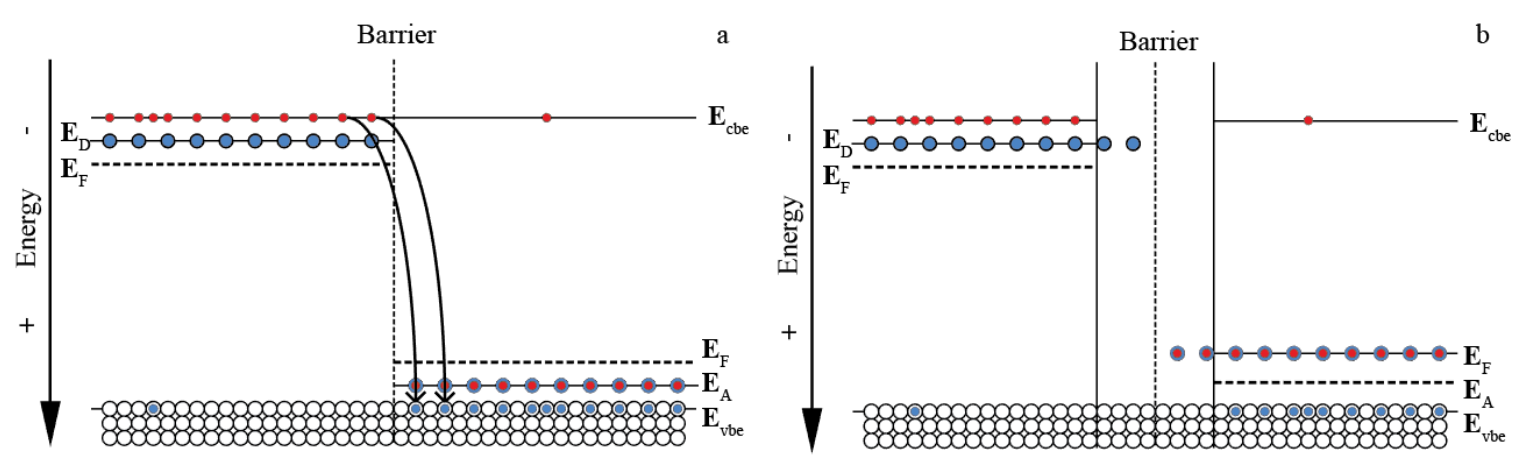

$00.0000,0000: 000000000$ $000000^{\circ} 000000000 \bullet 0000$ $000^{\circ} \circ 000000,00 \bullet 000000^{\circ} 0$ 00000.0000 of $0000 \bullet 0000$ $00.0000000010 \bullet 00000000$ $0000000.000000000 \bullet 000$ $00000.000000000 \cdot 00000$ $000.00000000000000 \bullet 00$ 000000.000 - 000000000 000000000 ólo 00000000 .

○ Constituent Atom (0)

- Free Electron $\left(\mathrm{e}^{-}\right)$

$\odot$ Constituent Atom, Ionized $\left(\mathrm{h}^{+}\right)$

o Donor Atom $\mathrm{N}_{\mathrm{D}}$, Ionized (+)

- Acceptor Atom $\mathrm{N}_{\mathrm{A}}$, Ionized (-)

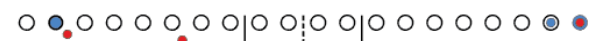

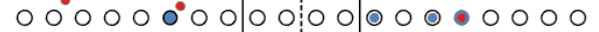

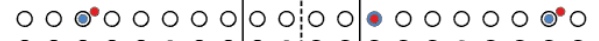
00000.00000 ० ० ० $000 \bullet 0000$ $00,00000000,0 \bullet 00000000$ $0000000.000,0000000000$ $00000.00000,0000-00000$

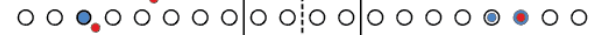

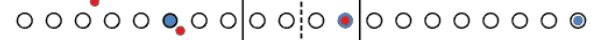

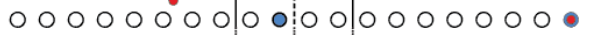
$-W_{\bar{D}}-W_{-}$

○ Constituent Atom (0)

- Free Electron (e-)

○ Constituent Atom, Ionized $\left(\mathrm{h}^{+}\right)$

- Donor Atom $\mathrm{N}_{\mathrm{D}}$, Ionized (+)

- Acceptor Atom $\mathrm{N}_{\mathrm{A}}$, Ionized (-)

Figure 1.3. Energy diagram of a $p-n$ junction. (a) is directly after the removal of the imaginary barrier. The black circles are the constituent atoms of the material. The red dots are free electrons. The black circles with blue dots in them are the constituent atoms that have lost an electron and now have a positive charge, this charge is known as a hole.

The blue dots with thick black outlines are donor atoms that have been ionized which now have a positive charge. The blue circles with red dots in them are acceptor atoms that have accepted electrons and are now negatively charged. $\mathbf{E}_{\mathrm{D}}$ and $\mathbf{E}_{\mathrm{A}}$ are the energy levels of the donors and acceptors. $\mathbf{E}_{\mathrm{cbe}}$ and $\mathbf{E}_{\mathrm{vbe}}$ are the energy levels of the conduction and valence band edges. (b) is directly after a when no more electrons can move from the n-type to the p-type. The Fermi level is also included on each side as a horizontal dotted line. The bottom half of both (a) and (b) are two dimensional physical representations of the atoms in each of the materials. In (b) the width $W$ is the depletion width and the widths $W_{\mathrm{D}}$ and $W_{\mathrm{A}}$ are the depletion widths on the donor and acceptor doped semiconductors.

Figure 1.3.a shows what happens directly after the removal of the barrier. The ntype side has a greater concentration of electrons, diffusion drives them into the p-type material. They are also at a more negative energy. The figure below the band diagram also shows the physical movement of the electrons from the n-type to the p-type. Figure 1.3.b shows what happens directly after this occurs. The n-type side has an area that has donated 
the electrons but is now left with the positive charges of the donor atoms. The p-type side has accepted the electrons into its positively charged constituent atoms leaving this area with a negative charge from the ionized acceptor atoms. This resulting charge creates an electric field whose direction drives electrons from the p-type to the n-type. At this moment the forces of the drift of the electric field encouraging movement of electrons into the ntype side and the diffusion encouraging electrons into the p-type side is equal and there is no net movement of electrons. This region at the junction is depleted of carriers, holes or electrons. Therefore, the region is called the depletion region. Its physical width is marked $W$ on Figure 1.3.b. The width on the n-type side which was doped with donors is designated $W_{\mathrm{D}}$, and the width on the p-type side, which was doped with acceptors, $W_{\mathrm{A}}$. The depletion width of each side is dependent on the concentration of donor atoms in the n-type material $\left(N_{\mathrm{D}}\right)$, the concentration of donor atoms in the p-type material $\left(N_{\mathrm{A}}\right)$, and the built-in voltage $\left(\Psi_{\mathrm{bi}}\right)$. The built-in voltage is the difference of energy of the Fermi levels of the $\mathrm{p}$ and $\mathrm{n}$ type side before removal of the barrier. At equilibrium the Fermi level is constant throughout the material. This is not the case in Figure 1.3. What makes it so that the Fermi level is constant throughout is the field in the depletion region. 

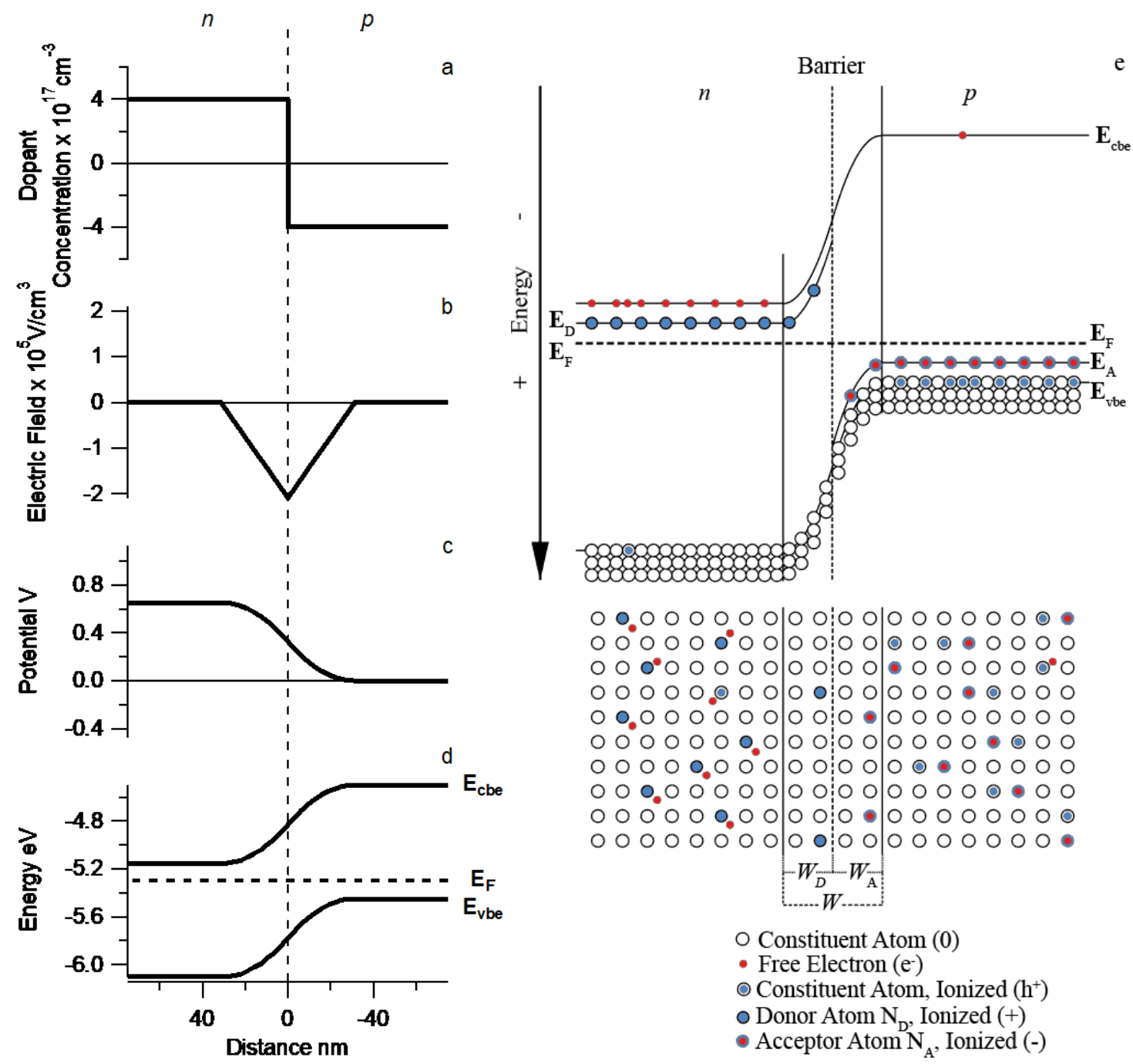

○ Constituent Atom ( 0$)$

- Free Electron (e-)

- Constituent Atom, Ionized $\left(\mathrm{h}^{+}\right)$

○ Donor Atom $\mathrm{N}_{\mathrm{D}}$, Ionized (+)

- Acceptor Atom $\mathrm{N}_{\mathrm{A}}$, Ionized (-)

Figure 1.4 (a) is the concentration of donors at the interface of a $p$ - $n$ junction where the $\mathrm{n}$ type is at positive distance and p-type at a negative, (b) the electric field built up at the same location, (c) the potential built up at the same location, (d) the band bending at the same location. (e) is the energy band diagram resulting from band bending caused by the electric field. The black circles are the constituent atoms of the material. The red dots are free electrons. The black circles with blue dots in them are the constituent atoms that have lost an electron and now have a positive charge, this charge is known as a hole. The blue dots with thick black outlines are donor atoms that have been ionized which now have a positive charge. The blue circles with red dots in them are acceptor atoms that have accepted electrons and are now negatively charged. $\mathbf{E}_{\mathrm{D}}, \mathbf{E}_{\mathrm{A}}, \mathbf{E}_{\mathrm{cbe}}, \mathbf{E}_{\mathrm{vbe}}$ and $\mathbf{E}_{\mathrm{F}}$ are the energy levels of the donors, acceptors, conduction band edge, valence band edge and Fermi level. The bottom half of (e) is a two-dimensional physical representation of the atoms in each of the junction. $W$ is the depletion width and the widths $W_{\mathrm{D}}$ and $W_{\mathrm{A}}$ are the depletion widths on the donor and acceptor doped semiconductors. 
To determine the electric field generated in the depletion regions the dopant concentrations must be known. In this example the dopant concentration is constant through the $\mathrm{n}$ and p-type materials. This is shown in Figure 1.4.a. The resulting electric field is calculated by:

$$
\varepsilon_{D}=\frac{q N_{D}}{\varepsilon_{S}} W_{D}, \varepsilon_{A}=\frac{-q N_{A}}{\varepsilon_{S}} W_{A}
$$

Where $q$ is the elementary charge, $N_{D}$ is the donor density, $N_{\mathrm{A}}$ is the acceptor density $\varepsilon_{\mathrm{S}}$ is the static dielectric constant of the semiconductor, and $W_{\mathrm{A}}$ and $W_{\mathrm{D}}$ are the widths from the junction. The resulting electric field calculations across the depletion region are shown in Figure 1.4.b. The electric potential in the depletion region is calculated by:

$$
V=\frac{q N_{D}}{2 \varepsilon_{S}} W_{D}^{2}, V=\frac{-q N_{A}}{2 \varepsilon_{S}} W_{A}^{2}
$$

This is calculated and shown for the depletion region in Figure 1.4.c. Adding the electric potential changes to the appropriate sides of the junction results in Figure 1.4.d. Applying this to the previous diagram results in Figure 1.4.e. This is the resulting band diagram and physical representation of the junction of the $\mathrm{p}$ and n-type semiconductor. This is the diagram in the dark, discussed next is what happens when this is illuminated. 

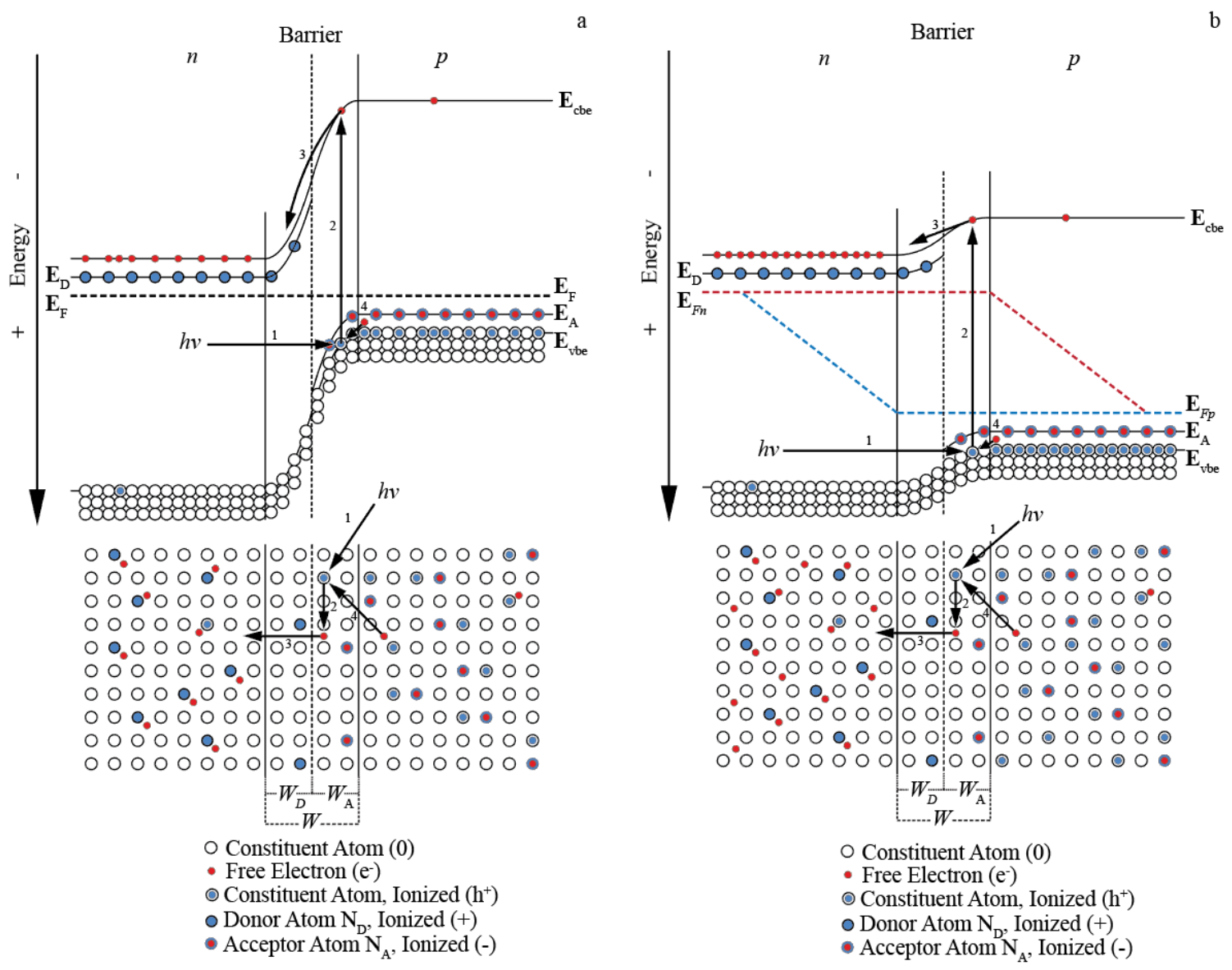

Figure 1.5. $(\mathrm{a}, \mathrm{b})$ energy band diagram of $p$ - $n$ junction. The black circles are the constituent atoms, the red dots are free electrons, the black circles with blue dots in them are constituent atoms that have lost an electron and now have a positive charge, and this charge is known as a hole. The blue dots with thick black outlines are donor atoms that have been ionized which now have a positive charge. The blue circles with red dots in them are acceptor atoms that have accepted electrons and are now negatively charged.

$\mathbf{E}_{\mathrm{D}}, \mathbf{E}_{\mathrm{A}}, \mathbf{E}_{\mathrm{cbe}}, \mathbf{E}_{\mathrm{vbe}}$ and $\mathbf{E}_{\mathrm{F}}$ are the energy levels of the donors, acceptors, conduction band edge, valence band edge and Fermi level. The bottom half of $(a, b)$ is a two dimensional physical representations of the atoms in each of the junction. $W$ is the depletion width and

the widths $W_{\mathrm{D}}$ and $W_{\mathrm{A}}$ are the depletion widths on the donor and acceptor doped semiconductors. (a) is at the introduction of the first photon with energy equal to the band gap. This photon labeled $h v$ is introduced into the junction which is labeled as arrow 1.

The photon is absorbed by an electron and promoted to the conduction band this is labeled as arrow 2. The electron is then carried by the drift of the electric field into the ntype side of the semiconductor labeled arrow 3 . The hole created in the conduction band by step 2 is then combined with an electron from the valence band from the p-type side labeled arrow 4. This is also shown in (b) which is after the net movement of electrons is zero. (b) does not have a fermi level but two quasi fermi levels labeled $\mathbf{E}_{\mathrm{Fn}}$ and $\mathbf{E}_{\mathrm{Fp}}$. 
If the semiconductor has the appropriate properties, incident light upon the material is absorbed by electrons. If the energy of the light is equal to or greater than the conduction band, the electron absorbing the photon is promoted to or above the conduction band. This is shown in Figure 1.5.a. First light hits the material labeled by arrow 1. This is absorbed by an electron which is promoted to the conduction band as shown by arrow 2 . This creates a hole in the valence band edge. The electron is then within the depletion region where the drift created by the field moves it to the n-type material labeled arrow 3. An electron in the p-type material can fall into the vacancy labeled arrow 4. It is common to think of this as holes moving into the p-type material. This same process is depicted underneath in the physical representation. Overall the light is giving the electrons the energy for promotion into the conduction band and the electric field moves and keeps them in the n-type side and electrons from the p-type side replenish the electrons in the depletion region. This unbends the bands as is depicted in Figure 1.5.b. This reaches a limit where it is no longer favorable to increase the concentration of electrons in the n-type and holes in the p-type. The resulting energy band and physical diagram is shown in Figure 1.5.b. This is no longer in equilibrium, so the Fermi level is not constant throughout the material. Here there is a quasi-Fermi level of electrons on the n-type side and quasi Fermi level of electrons in the p-type side labeled $\mathbf{E}_{\mathrm{Fn}}$ and $\mathbf{E}_{\mathrm{Fp}}$. The difference in these two Fermi levels is the potential difference of the two sides of the semiconductor. The measurement of this difference when there is no external circuit is the open circuit photovoltage, $V_{\mathrm{ph}}$, the quantity of interest when it comes to pyrite as a photovoltaic. This is ideally only limited by the built-in voltage $\left(\Psi_{\mathrm{bi}}\right)$ and thermodynamic considerations. If the two sides of the junction are directly 
electrically connected the current running through it is the short circuit current $\left(I_{\mathrm{sc}}\right)$. In this case electrons can leave the n-type material externally and return to the p-type material so that there is no reversal of the band bending and no observed photovoltage. This is how common solar cells work and with advances through research pyrite could be used as the semiconductor material shown in the previous diagrams as a solar cell material.

\subsection{Pyrite as a solar cell material}

The realization of cost-competitive solar-to-electrical energy conversion will revolutionize a range of important issues spanning the environment to national security. Iron pyrite $\left(\mathrm{FeS}_{2}\right)$ is a promising material component for solar-to-electrical energy conversion. On paper, pyrite is a clear winner in the search for an earth-abundant photovoltaic absorber because of its advantageous properties as an ideal component in multi-junction solar cells. Pyrite has an optical bandgap of $0.95 \mathrm{eV}$ with an electronic bandgap of $0.80 \mathrm{eV},{ }^{1}$ which result in theoretically high efficiencies. ${ }^{1,2}$ Pyrite crystallized from various synthetic routes have shown high absorption coefficients $\left(>6 \times 10^{5}\right.$ for $h v>$ $1.3 \mathrm{eV}$ ), resulting in the need for very thin films $<200 \mathrm{~nm}$ for complete absorption. ${ }^{3,4}$ Pyrite has also shown photon to electron conversion efficiencies of over $90 \%$ resulting in the possibility of a majority of absorbed light resulting in electrical current. ${ }^{5,3,6,7}$ Elemental iron and sulfur are the $4^{\text {th }}$ and $17^{\text {th }}$ most abundant element in the earth's crust. ${ }^{8}$ In 2014 there were 3,200 and 72.4 million metric tons of iron and sulfur mined globally. ${ }^{9}$ In 2014 the cost of iron and sulfur were each only 10 cents $/ \mathrm{kg} .{ }^{9}$ Considerations of extraction cost and theoretical power maximum have determined pyrite to have the theoretical ability to provide more than 10,000 times the annual worldwide power consumption. ${ }^{10}$ Pyrite is 
nontoxic. These properties position pyrite as an ideal component in multi-junction solar cells.

Furthermore, near unity (0.923) photon-to-electron conversion efficiency under intense illumination $\left(4-5 \mathrm{~W} \mathrm{~cm}^{-2}\right)$ has been reported for monocrystalline n-type $\mathrm{FeS}_{2}$ in contact with an aqueous solution containing an $\mathrm{I}_{3}{ }^{-} / \mathrm{I}^{-}$redox couple. ${ }^{7}$ The reported chargecarrier diffusion lengths $(0.1-1 \mu \mathrm{m})$ match the materials thickness required based on the extinction coefficient of $\mathrm{FeS}_{2}$. ${ }^{11-16}$ There is also some evidence that polycrystalline $\mathrm{FeS}_{2}$ may be relevant for solar-to-electrical energy conversion. ${ }^{17}$

Both single and polycrystalline samples of pyrite have been made into electrodes by contacting the crystal to a Ga-In alloy contacted to a wire with silver in an encased housing and having their photocurrents measured in a cell vs. $\mathrm{Hg}_{2} \mathrm{SO}_{4}$ in solutions of $4-5 \mathrm{M}$ KI against a range of monochromatized light in order to determine their quantum efficiencies which had values of up to over $90 \% .^{5,3,6,7}$

\subsubsection{Challenges for pyrite solar cells}

Despite all the favorable properties of pyrite, many challenges remain. All pyritebased photovoltaic and photoelectrochemical cells have exhibited low photovoltages $\left(V_{\mathrm{ph}}\right.$ $<200 \mathrm{mV}$ ). Thermodynamic considerations predict photovoltages of pyrite to be well over values experimentally published. The maximum $V_{\mathrm{ph}}$ obtainable for a semiconductor is reduced by the temperature difference of the sun and the cell as well as entropic losses due to radiative recombination amounting to $315 \mathrm{mV}$ at most. ${ }^{2,11,12,13}$ This results in the maximum $V_{\text {ph }}$ of pyrite being over $400 \mathrm{mV}$. However, nothing of this magnitude has been reported so far. 
Polycrystalline thin films have been grown onto $\mathrm{TiO}_{2}$ utilizing metalorganic chemical vapor deposition (MOCVD) from iron pentacarbonyl and sulfur. These resulted in a $V_{\mathrm{ph}}$ of $500 \mathrm{mV} \cdot{ }^{14}$ This is higher than the theoretical limit for pyrite and as a result must be reflecting the performance of a contaminant phase, or corrosion of the $\mathrm{FeS}_{2}$ material. Single crystals grown with CVT and Br have been made into Schottky junctions with various metals and were able to produce almost $50 \mathrm{mV}$ for their $V_{\mathrm{ph}} .{ }^{15}$ Polycrystalline layers of As doped pyrite grown by CVT with Br from a precursor growth with iodine included have shown photoactivity. This was done in a 3 electrode setup containing an electrolyte consisting of $1 \mathrm{M} \mathrm{CaI}_{2}$ and $0.05 \mathrm{M} \mathrm{I}_{2}$ and resulted in a $V_{\mathrm{ph}}$ of almost $80 \mathrm{mV} .{ }^{3}$ Synthetic $\mathrm{FeS}_{2}$ was made into an electrode and also tested in a 3 electrode setup but with an electrolyte of $3 \mathrm{M} \mathrm{KI}$ and $0.01 \mathrm{M} \mathrm{I}_{2}$ showing a $V_{\mathrm{ph}}$ of about $200 \mathrm{mV} .{ }^{6}$ Another synthetic pyrite electrode in an electrolyte of $4 \mathrm{M} \mathrm{HI}, 0.05 \mathrm{M} \mathrm{I}_{2}$ and $2 \mathrm{M} \mathrm{CaI}_{2}$ have reached a $V_{\mathrm{ph}}$ of $187 \mathrm{mV}$ with a solar to electrical conversion efficiency of $2.8 \%$ (photon to electron conversion efficiency, PCE). ${ }^{4,16}$

The $V_{\text {ph }}$ directly effects the PCE. The PCE is the ratio of power out of the cell to the power into the cell. The equation to determine electric power produced by a cell is shown below.

$$
P C E=\frac{V_{p h^{*} J_{S c} * F}}{P_{\text {in }}}
$$

$J_{\mathrm{sc}}$ is the short circuit current density, $F F$ is the fill factor. $P_{\text {in }}$ is the power of the light upon the cell and can be determined from the light source with a diode. To illustrate the effects of $F F$ on the current-voltage characteristics of a photovoltaic cell, consider Figure 1.6.a. 

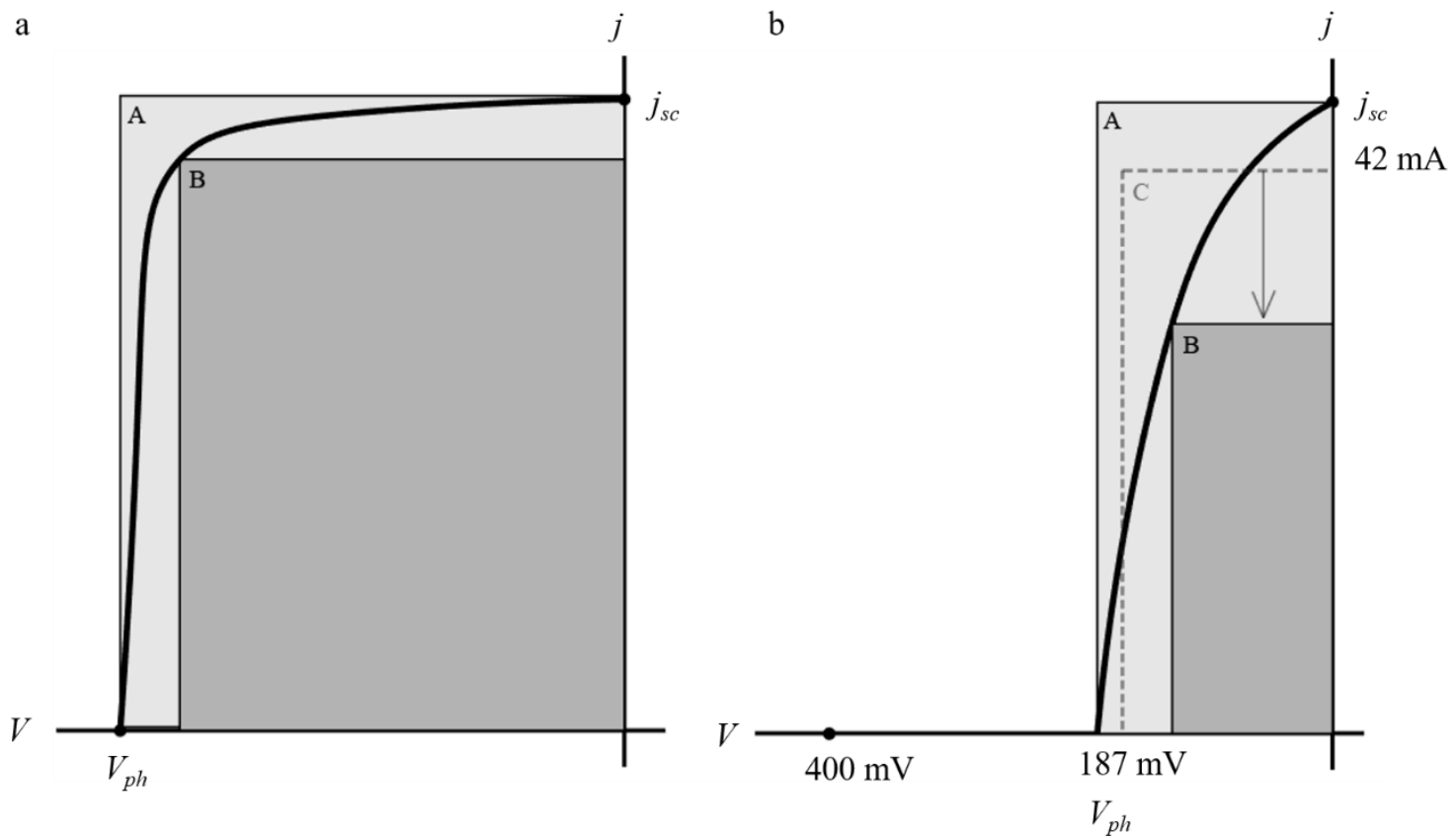

Figure 1.6. Current-voltage (I- $V$ ) characteristics of a typical photovoltaic cell with a good fill factor $(F F)$ (a) and typical I- $V$ curve for a $\mathrm{FeS}_{2} \mathrm{I}^{-} / \mathrm{I}_{3}{ }^{-}$cell (b) with lower $F F$ values.

Box A has the height of the short circuit current density $\left(j_{\mathrm{sc}}\right)$ and the width of the photovoltage $\left(V_{\mathrm{ph}}\right)$. At each point of the plot the product of the current and voltage is equal to the power that can be produced. Box B's height and width are determined from the max power point. In (a) box B corresponds to a $F F$ of 0.8 . In (b) box B corresponds to a $F F$ of 0.5 . In (b) box $C$ has a fill fact of 0.8 .

The figure is a current voltage (I- $V$ ) curve. The potential from one side of the junction to the other is altered while the current is measured. In this case this is done while light is shining onto the cell. There are two different boxes in the figure, A and B. Box A's height is equal to the short circuit current density. This is the current when $V_{\text {ph }}$ is zero. Box A's width is equal to the open circuit photovoltage, the photovoltage when $j_{\text {sc }}$ is zero. The product of the photovoltage and the photocurrent at each point on the curve produce a power. Plotting the power against the photovoltage result in a power curve. The point at which the maximum power is produced by a solar cell is called the maximum power point 
(MPP). Box B's height is the photocurrent at this point and its width is the photovoltage at this point. $F F$ is the ratio of the area of box $\mathrm{B}$ to the area of box $\mathrm{A}$.

The $F F$ is known to be affected by both series and shunt resistance ( $R_{\mathrm{ser}}$ and $\left.R_{\mathrm{sh}}\right)$. $R_{\text {ser }}$ is the resistance of the series of elements in the circuit. These include the resistance of the materials of the cell (the semiconductor and the metal carrying the current to and from the semiconductor), and the contacts of the cell (contact between metal and semiconductor). A high $R_{\text {ser }}$ can lower the photocurrent and can result in a linear shape of the current-voltage response of a solar cell. $R_{\text {ser }}$ does not affect the open circuit photovoltage value. $R_{\mathrm{sh}}$ is the resistance of shunts. Shunts are electric pathways connecting the $\mathrm{p}$ and $\mathrm{n}$-type semiconductors other than the depletion region. A low shunt resistance means that electrons can continue to travel from the n-type to the p-type without being stopped by the drift of the electric field created by the depletion region. This means that they are limited in their accumulation onto the n-type side of the junction and result in a diminishing of the band bending at the junction and lower $\Psi_{\mathrm{bi}}$ which in turn lowers $V_{\mathrm{ph}}$. The shunts to the junction do not affect the short circuit current.

Figure 1.6.a. represents an idealized I- $V$ response of a cell with a high $F F$, about 0.8. Figure 1.6.b. is representative of pyrite based photovoltaic device. The $F F$ for pyrite is lower, about 0.5 . As shown in equation 3, with a higher $V_{\mathrm{ph}}, J_{\mathrm{sc}}$, and $F F$ the PCE is higher. The experimental values for $J_{\mathrm{sc}}$ measured for pyrite devices are high. Increasing the value of $F F$ from 0.5 to the realistically attainable value of 0.8 would increase the PCE by 66\%. Box C in Figure 1.6.b. represents the $F F$ at 0.8 for the same open circuit photovoltage and short circuit current. The largest gain would be in the open circuit photovoltage. This 
is currently around $187 \mathrm{mV}$ and can theoretically get past $400 \mathrm{mV}$. Doing so would result in more than doubling the PCE! This is the parameter in need of research focus.

It is reasonable that before making a $p$ - $n$ junction device, the semiconductor material itself and its $p$ - and $n$-type doped forms need to be characterized to allow for better understanding of the charge involving processes. This is typically done by making a similar junction with either metals (Schottky junction) or with redox containing liquid solutions. Each of these have their own advantages and have been used to characterize pyrite. The details of these junctions are discussed next. 


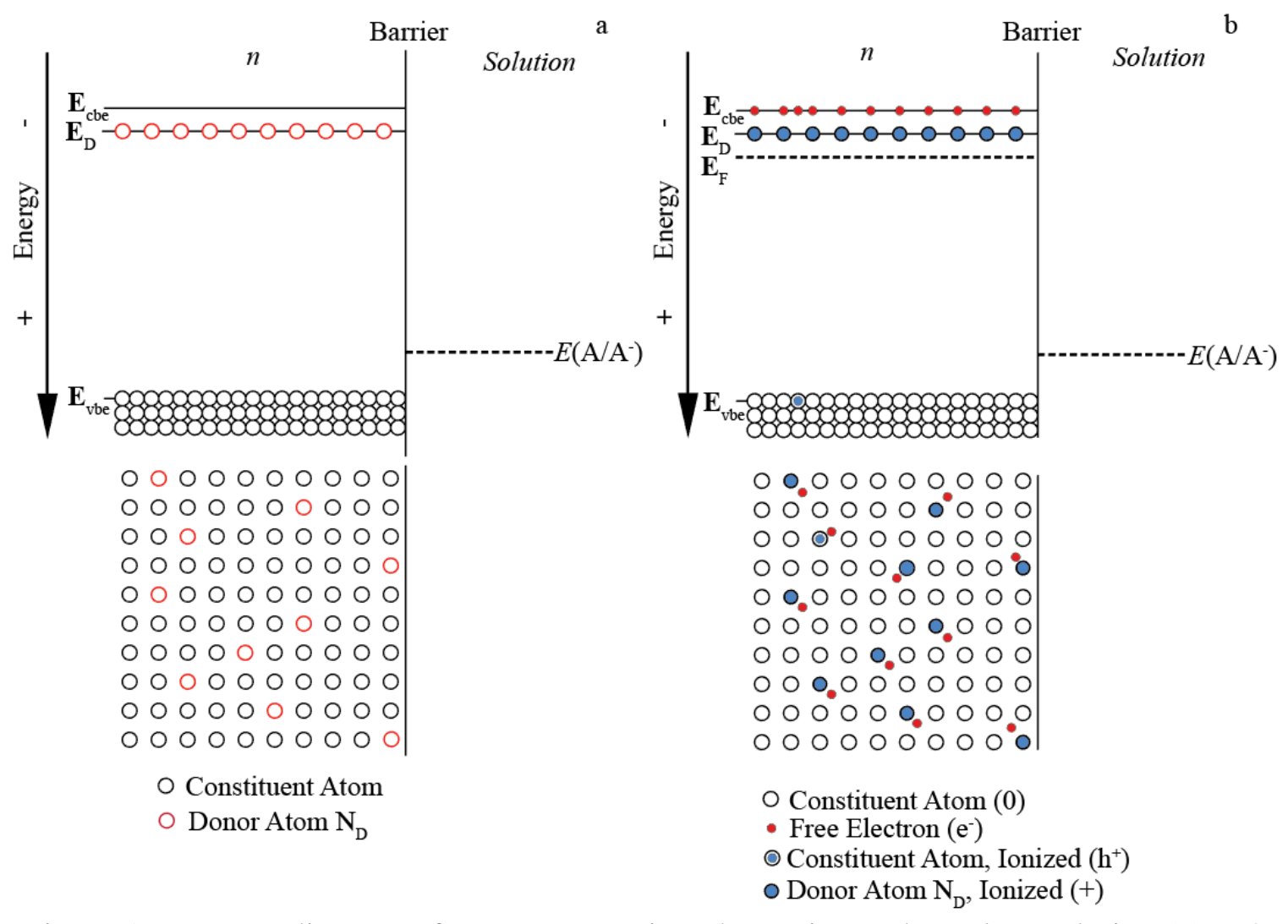

Figure 1.7 Energy diagram of an n-type semiconductor in an electrolyte solution (a) at 0 $\mathrm{K}$ and (b) at room temperature. The bottom half of both (a) and (b) are two dimensional physical representations of the atoms in each of the materials. The black circles are the constituent atoms of the material. The red circles are donor atoms. $\mathbf{E}_{\mathrm{D}}, \mathbf{E}_{\mathrm{cbe}}, \mathbf{E}_{\mathrm{vbe}}, \mathbf{E}_{\mathrm{F}}$, and

$E\left(\mathrm{~A} / \mathrm{A}^{-}\right)$are the energy levels of the donors, conduction and valence band edges, the Fermi level, and the solution potential. The atoms are labeled in the same way in $\mathrm{b}$ with the addition of free electrons as red dots, ionized constituent atoms as black circles with blue dots in them. Ionized donor atoms as blue dots with a black rim.

As shown in Figure 1.7.a. the energy diagram of an n-type semiconductor separated from an electrolyte solution looks like the $p-n$ junction. In this case at $0 \mathrm{~K}$ and room temperature this would look the same as it had in the $p-n$ junction in Figure 1.2.a. The $\mathrm{p}$ side is replaced with an electrolyte solution whose potential is determined by the standard potential of the redox couple and the concentration of each species according to the Nernst equation: 


$$
E\left(A / A^{-}\right)=E^{\circ}\left(A / A^{-}\right)+\frac{k T}{n_{e}} \ln \frac{[A]}{\left[A^{-}\right]}
$$

Where $E\left(A / A^{-}\right)$is the solution potential, $E^{\circ}\left(A / A^{-}\right)$is the standard redox potential, $k$ is the Boltzmann constant, $T$ is temperature, $\mathrm{n}_{\mathrm{e}}$ is the stoichiometric number of electrons involved in the redox reaction, $[\mathrm{A}]$ is the concentration of oxidized species and $\left[\mathrm{A}^{-}\right]$is the concentration of reduced species. The solution can accept electrons by reducing the oxidized species and donates electrons by oxidizing the reduced species. This is a great tool as different electrolyte couples have different standard redox potentials and by changing the concentration of oxidized and reduced species the solution potential can be fine-tuned. Metals can also be used to make a junction and instead of the solution potential the work function of the metal would determine its energy level. The initial diagram of the solution contact with a barrier present is shown in Figure 1.7.a. Figure 1.7.a is at $0 \mathrm{~K}$ and Figure 1.7.b at room temperature. At room temperature the electrons from the donor atoms are promoted into the conduction leaving behind a positive charge. 


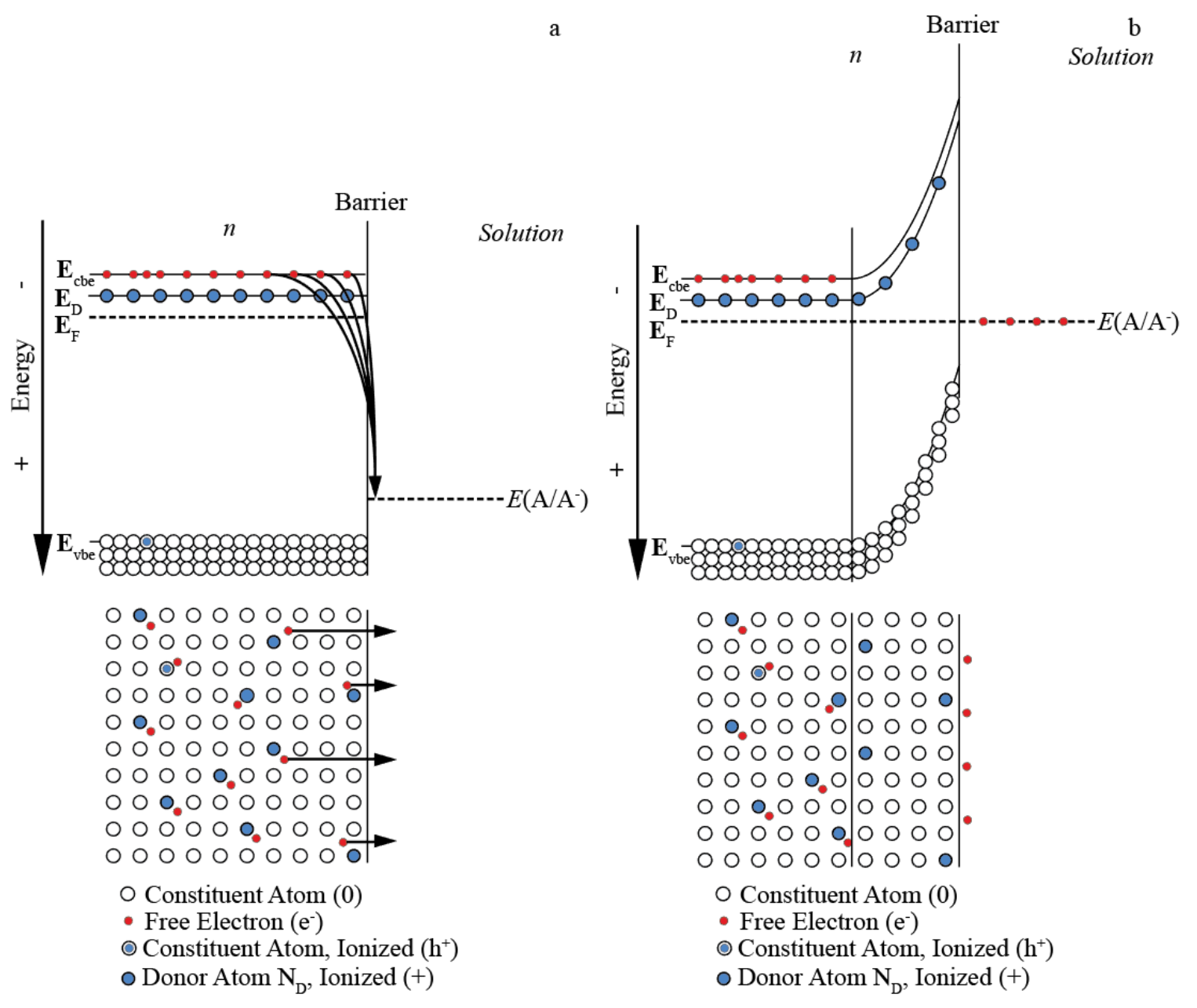

Figure 1.8 Energy diagram of an n-type semiconductor in an electrolyte solution. (a) is right after the barrier between the two is removed. The black circles are the constituent atoms of the material. The red dots are free electrons. The black circles with blue dots in them are ionized constituent atoms. The blue dots with thick black rims are ionized donor atoms. $\mathbf{E}_{\mathrm{D}}, \mathbf{E}_{\mathrm{cbe}}, \mathbf{E}_{\mathrm{vbe}}, \mathbf{E}_{\mathrm{F}}$, and $E\left(\mathrm{~A} / \mathrm{A}^{-}\right)$are the energy levels of the donors, conduction and valence band edges, the Fermi level, and the solution potential. The atoms are labeled in the same way in (b) (b) is at equilibrium when the electrons from the n-type semiconductor are no longer able to move into the solution due to the buildup of the electric field at the junction. The bottom half of both (a) and (b) are two dimensional physical representations of the atoms in each of the materials.

Upon removal of the barrier, electrons of a more negative energy move from the ntype semiconductor into the solution as shown in Figure 1.8.a. The electrons then reduce the oxidized species of the electrolyte solution. Similar to the $p$ - $n$ junction, the n-type 
semiconductor losing electrons leaves a positive charge on the donor atoms. Balancing this charge is the reduced species of the redox couple which is attracted to the positive charge of the donor atoms and remain at the junction surface. This creates a field similarly to the $p-n$ junction, however in this case all the field is in the semiconductor. The resulting energy band diagram is shown in Figure 1.8.b. The width of this depletion region is now determined by the difference of the electrochemical potential of the semiconductor and the solution (Fermi level and redox potential) and donor concentration $N_{\mathrm{D}}$. The Fermi level of the semiconductor is determined by identity and its doping and the electrochemical potential of the solution is determined by its identity and the concentration of reduced and oxidized species according to the Nernst equation. The ability to change the solution potential allows for the largest built in potential, which maximizes $V_{\mathrm{ph}}$. 

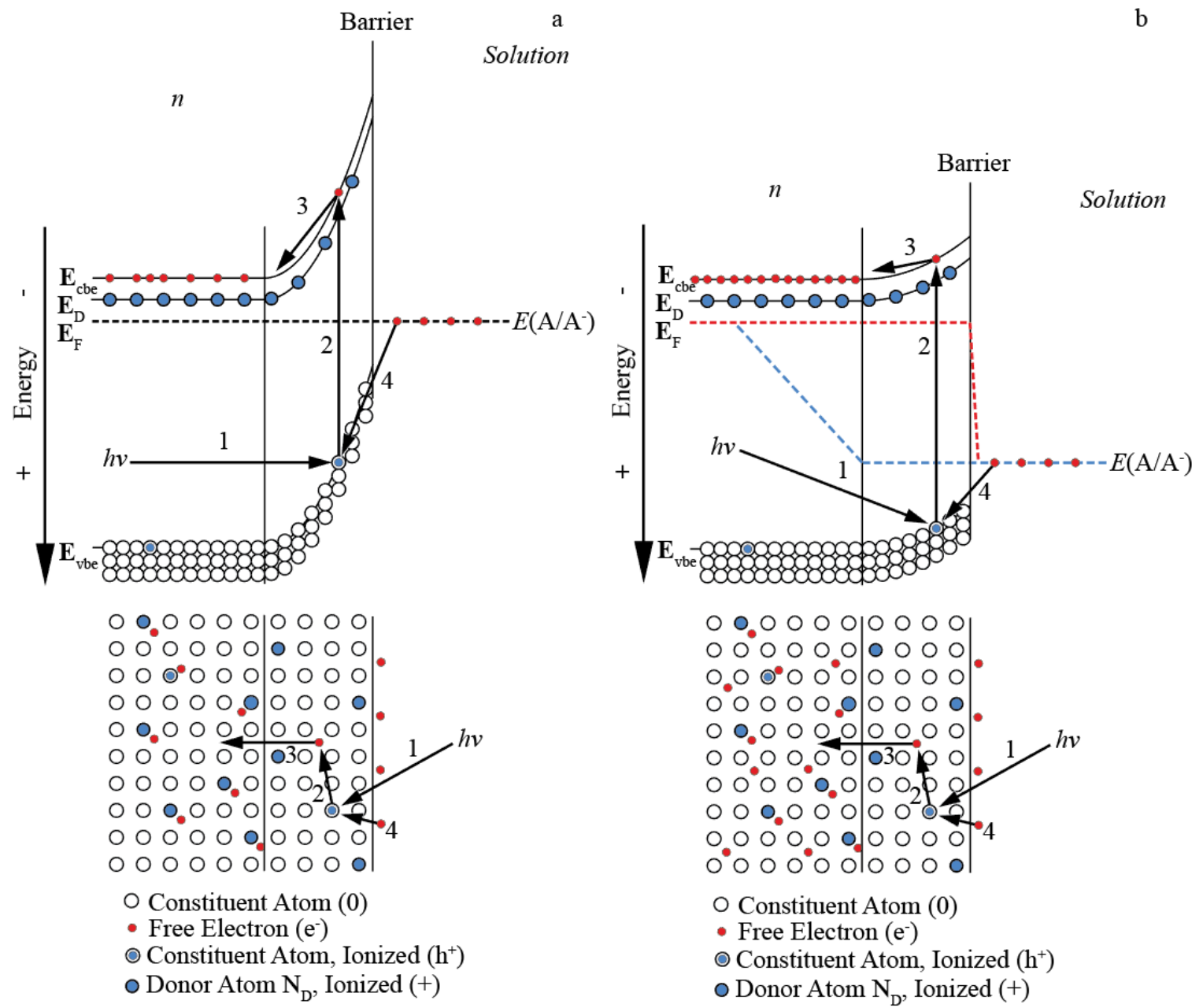

Figure 1.9 Energy diagram of an n-type semiconductor in an electrolyte solution. The black circles are the constituent atoms of the material. The red dots are free electrons. The black circles with blue dots in them are ionized constituent atoms. The blue dots with thick black rims are ionized donor atoms. $\mathbf{E}_{\mathrm{D}}, \mathbf{E}_{\mathrm{cbe}}, \mathbf{E}_{\mathrm{vbe}}, \mathbf{E}_{\mathrm{F}}, E\left(\mathrm{~A} / \mathrm{A}^{-}\right), \mathbf{E}_{\mathrm{Fn}}$ and $\mathbf{E}_{\mathrm{Fp}}$ are the energy levels of the donors, conduction and valence band edges, the Fermi level, the solution potential, the quasi Fermi level of electrons, and the quasi Fermi level of holes.

(a) is at the introduction of the first photon with energy equal to the band gap. This photon labeled $h v$ is introduced into the junction which is labeled as 1 . The photon is absorbed by an electron and promoted to the conduction band this is labeled as 2 . The

electron is then carried by the drift of the electric field into the n-type side of the semiconductor labeled 3 . The hole created in the conduction band by step 2 is then combined with an electron from the valence band from the p-type side labeled 4 . This is also shown in (b) which is after the net movement of electrons is zero.

In a similar fashion to the $p$ - $n$ junction light hits the semiconductor and promotes electrons into the conduction band which are separated from the depletion region further 
into the semiconductor by the electric field. This reaches a limit for where the potential difference of the quasi Fermi levels is the measured open circuit photovoltage. This is shown in Figure 1.9. The ability to use this method can be greatly affected by Fermi level pinning which is a common scapegoat for low pyrite $V_{\mathrm{ph}}$.

\subsubsection{Fermi level pinning}

The band structure of semiconductors results from the periodicity of the lattice as explained in section 1.2. At the surface the lattice ends. It is reasonable to believe that the resulting electronic structure on the surface of the semiconductor is different form the bulk. If the resulting surface structure has energy levels that are within the bandgap it could form a junction with the bulk. Typical doping of a semiconductor introduces the dopant at concentrations around $10^{18} \mathrm{~cm}^{-3}$. When the material is of a density of $10^{22} \mathrm{~cm}^{-3}$ the dopant density is small enough that each dopant is far enough apart that it does not form a band with other dopant atoms but lies at a single energy level. The surface is of a greater density and result is the formation of a band. 


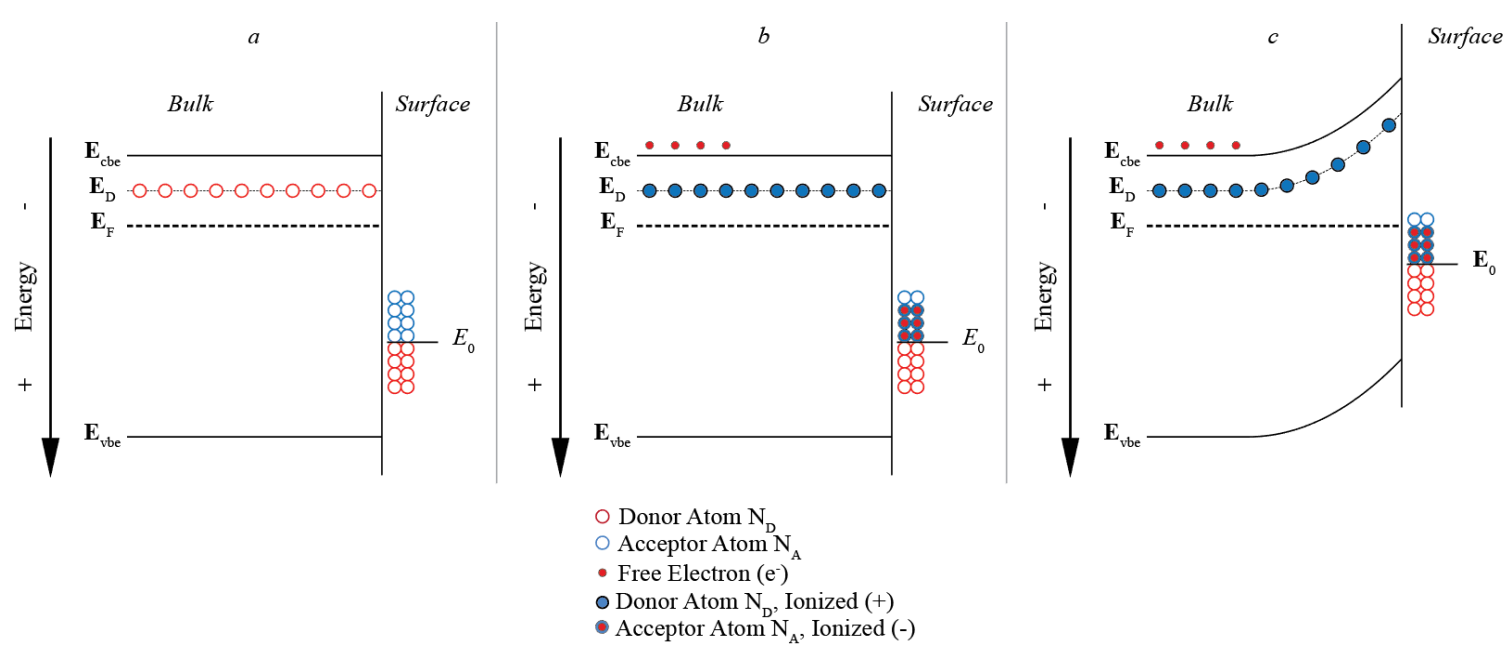

Figure 1.10 Formation of depletion region due to metallic band surface states. (a) at $0 \mathrm{~K}$, (b) after equilibrium, (c) depletion region formation. Red circles are dopant atoms, blue circles are acceptors. Red dots are free electrons, blue dots with black rims are ionized donor atoms, red dot with blue rims are ionized acceptor atoms. $\mathbf{E}_{\text {cbe }}, \mathbf{E}_{\mathrm{D}}, \mathbf{E}_{\mathrm{F}}, \mathbf{E}_{\mathrm{vbe}}, \mathbf{E}_{0}$ are the energy levels of the conduction band edge, donor atoms, fermi level, valence band edge and charge neutrality.

If this band resembles a metal, then it is only partially filled, and the Fermi level is within the band. The level to which it is filled is known as the charge neutral level $\mathbf{E}_{0}$. The energy states below this level belong to atoms which behave as donors, when they are filled they are neutral but when missing an electron, they are positively charged. The energy levels above $\mathbf{E}_{0}$ act as acceptors, when they are empty they are neutral and when an electron occupies them they have a negative charge. If this level is at a more positive level than the Fermi level of the bulk of the semiconductor then electrons from the semiconductor occupy the acceptor levels above $\mathbf{E}_{0}$. This process creates a depletion region resulting in the formation of an electric field, as it is shown in Figure 1.10. 


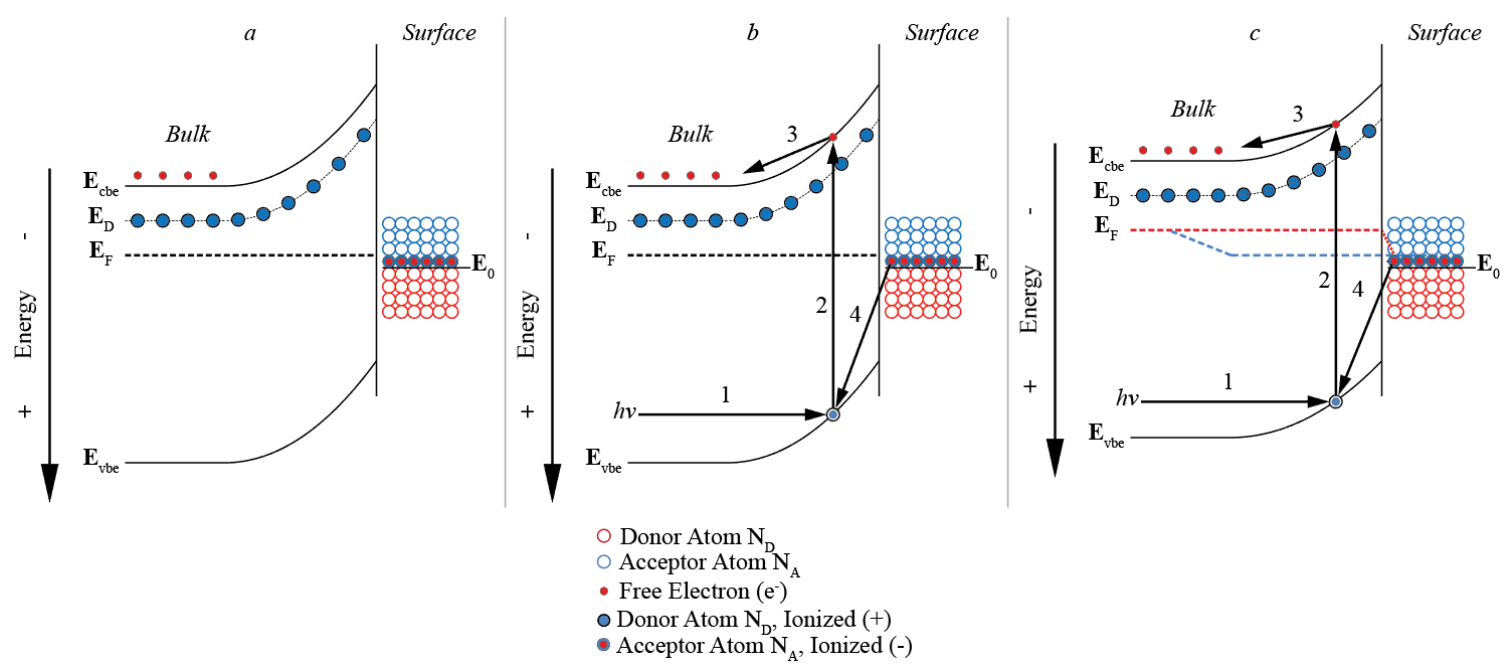

Figure 1.11 Energy diagram of junction of n-type material and its surface layer. (a) at equilibrium, (b) first incident photon, (c) at the limit of charge separation. Red circles are dopant atoms, blue circles are acceptors. Red dots are free electrons, blue dots with black rims are ionized donor atoms, red dot with blue rims are ionized acceptor atoms. $\mathbf{E}_{\mathrm{cbe}}, \mathbf{E}_{\mathrm{D}}$, $\mathbf{E}_{\mathrm{F}}, \mathbf{E}_{\mathrm{vbe}}, \mathbf{E}_{0}$ are the energy levels of the conduction band edge, donor atoms, fermi level, valence band edge and charge neutrality. (b) is at the introduction of the first photon with energy equal to the band gap. This photon labeled $h v$ is introduced into the junction which is labeled as 1 . The photon is absorbed by an electron and promoted to the conduction band this is labeled as 2 . The electron is then carried by the drift of the electric field into the n-type side of the semiconductor labeled 3 . The hole created in the conduction band by step 2 is then combined with an electron from the valence band from the p-type side labeled 4. This is also shown in (c) which is after the net movement of electrons is zero.

Figure 1.11.a shows the energy band diagram of the bulk and surface of a n-type semiconductor that has formed a depletion region. The built-in voltage is determined by the junction of the bulk and the surface. It does not matter what the potential is of the electrolyte solution contacting the surface, the photovoltage will be a result of this junction. The equation for the charge on the surface states is:

$$
Q_{s s}=-q D_{i t}\left(E_{F}-E_{0}\right)
$$

Where $q$ is the elementary charge, $D_{\mathrm{it}}$ is the density of interface states, $\mathbf{E}_{\mathrm{F}}$ is the Fermi level and $\mathbf{E}_{0}$ is the charge neutral level. The equation for the charge in the depletion region is: 


$$
Q_{s c}=q N_{D} W_{D}
$$

Where $N_{\mathrm{D}}$ is the donor concentration and $W_{\mathrm{D}}$ is the depletion width. For this surface the charges are equal:

$$
q N_{D} W_{D}=-q D_{i t}\left(E_{F}-E_{0}\right)
$$

For an n-type semiconductor of constant dopant concentration and depletion width, an increase in the density of interface states decreases the difference of the energy of the Fermi level and the charge neutral level. With the density of interface states reaching infinity the difference of the two energies is zero. This is how the Fermi level is pinned to the charge neutral level. The higher the density of surface states the more severe the pinning. This is one of the most common phenomenon that is blamed for the poor photovoltage of pyrite. However, both bulk and surface defects are implicated in limiting the $V_{\mathrm{ph}}$.

\subsubsection{Surface defects}

The surface acts as the gateway for electrons to transport to and from pyrite. If the surface is covered in defects that have a work function or Fermi level relatively close to the Fermi level of pyrite they will limit the pyrite's band bending which is directly correlated to the $V_{\mathrm{ph}}$. A Pt/FeS $S_{2}$ interface was determined to have Fermi level pinning from mid-gap states which probably arrive due to a sulfur vacancy. ${ }^{15}$ Synthetic pyrite in an electrochemical cell utilizing different redox solutions gave rise to the same photovoltage indicating that the band bending is due to the interface of pyrite with something other than the redox species and is most likely pinning due to the defect surface states of pyrite. ${ }^{17}$ XPS has been used to create a ligand field model describing the creation of states on the surface created by altering the symmetry of pyrite from $\mathrm{O}_{\mathrm{h}}$ to $\mathrm{C}_{4 \mathrm{v}}$ at the surface. This new surface 
layer pins the Fermi level. ${ }^{18}$ Measured temperature dependent surface photovoltages determined that modeling of the charge carrier dynamics at the surface could only be explained with mid-gap states pinning the Fermi level. ${ }^{19}$ DFT calculation showing a cleaving of pyrite leaving the Fe-S bond at the surface creating a new states leading to Fermi level pinning. ${ }^{20}$ STM together with DFT calculations predict that surface defects of sulfur or iron vacancies induce states within the bandgap pinning the Fermi level. ${ }^{21} \mathrm{~A}$ high density of surface states revealed by a gated hall effect technique is the blame for Fermi level pinning. ${ }^{22}$ Defects on the surface of both polished and cleaved surfaces imply that the same defects affecting the surface could affect the bulk and should be considered problematic. ${ }^{23}$ Calculation efforts towards determining crystal defects and impurities found that iron and sulfur vacancies have large formation enthalpies and induce defect states with high ionization energies $(>0.3 \mathrm{eV})$ which cannot provide enough free carrier density in pyrite. ${ }^{24}$

The photoactivity of pyrite has been improved by surface treatments. Acids have been used successfully but they tend to dissolve pyrite although this has been shown to depends on the $\mathrm{pH}$ of the solution. ${ }^{23,25}$ The corrosion of the surface has been reduced by layers formed from silanes and humic acids or by halogens and coordinating groups. ${ }^{26-29}$

Both bulk ${ }^{19,30}$ and surface ${ }^{1,22}$ chemistry are thought to limit the photovoltaic performance of $\mathrm{FeS}_{2}$ absorbers; mid-gap states and band-edge tail states both generally affect observed photovoltages. ${ }^{18,22,31,32}$ Furthermore, scanning tunneling spectroscopy results have shown that the surface bandgap is significantly lower than the bulk band gap $(0.4 \pm 0.1 \mathrm{eV}) .{ }^{21}$ Recent DFT-GGA calculations have shown that the bandgap of an $\mathrm{FeS}_{2}$ 
monolayer decreases to $0.73 \mathrm{eV} .^{33}$ However, it is not well understood how surface chemistry is connected to observed photovoltaic figures of merit, including photovoltages, even though it is well known that surface chemical treatments do affect photoelectrochemical response.

\subsubsection{Bulk defects}

Many of the literature references blame not only surface defects but bulk as well for the low photovoltage of pyrite. A variety of techniques were used to show that the acceptor states at the surface of pyrite pin the Fermi level to the surface near the valence band as was previously described but that this was not the main cause of the low photovoltage, the bulk ionization of deep donor states was the determined scapegoat. ${ }^{30}$ Bulk defects limit the $V_{\text {ph }}$ by creating defect states within the bandgap that serve as intermediate steps for holes to populate through thermal conduction allowing non-radiative recombination with electrons stopping them from contributing to the $V_{\mathrm{ph}}$. Because hydrogen can enter the interior of pyrite, the bulk can also be considered as an area improvable by hydrogen insertion. The insertion of hydrogen changes FeS centers with dangling bonds that serve as recombination centers within the bandgap into $\mathrm{FeSH} .{ }^{34,28}$

\subsubsection{Moving forward}

Surface and bulk defects are thought to limit photovoltage. The overall challenge is to modify pyrite bulk and surface and determine if there is a change to its photovoltage. This challenge is met by the thesis of this work: Pyrite's growth method and surface modification can be correlated to its photoelectrochemistry. Specifically: 1) $\mathrm{FeS}_{2}$ can be grown by liquid phase epitaxy onto natural and synthetic substrates. This would result in a 
different thin film of synthetic pyrite. All attempts to grow thin films of pyrite that are not homoepitaxial have resulted in the creation of thin films made of many small crystals. The properties of these films have been dominated by the surfaces of the small crystals as opposed to the bulk film. Therefore, it is necessary to make a thin film of one crystal so that there is only one surface that can be modified and correlated to photovoltage. Modifying the surface of the top of a polycrystalline film may still reflect a photovoltage that suffers the effects of the surfaces of the crystals in the bulk beneath the surface. A homoepitaxial growth does not include the surface defects from the polycrystalline films within the bulk. 2) The photovoltaic properties of liquid phase epitaxial growth are substrate dependent, a higher quality substrate will result in a higher photovoltage. 3) The photovoltage of pyrite may be explained by the thickness of the inversion layer on the surface resulting from its surface treatment. The explanation for the low photovoltage of pyrite involves Fermi level pinning and a deep donor so that there is very thin depletion region at the surface which allows electrons to tunnel across and limit the photovoltage. The thickening of this surface layer limits the tunneling so that the photovoltage is limited to the junction of the surface layer and the bulk and not from the bulk to the solution junction. 


\section{Crystalline growth of synthetic pyrite onto a natural substrate}

A review of the literature of different pyrite growth methods is required before selecting an appropriate growth technique. The method of growth must aim to limit the sulfur vacancies in the material by providing a high activity of sulfur throughout the growth. This can be done by including an excess of sulfur to create a sulfur rich growth environment. A specific set of instructions for said growth must then be determined and carried out. Confirmation of the epilayer is of high significance. Once this is complete the measurement of the photovoltage is conducted to assess if it has changed due to the growth.

\subsection{Introduction}

\subsubsection{The challenges of pyrite synthesis}

Sulfur vacancies have emerged as the most pressing chemical challenge limiting $V_{\mathrm{ph}}$ of pyrite. This is the case for both the surface and the bulk of pyrite. The proposed growth method utilizes a significantly higher $\mathrm{S}$ activity than that of sulfur in the chemical vapor transport (CVT) technique used to crystalize pyrite that has shown photoactivity. High sulfur activity meaning the scientific term of a high effective concentration, in this case provided by an excess of sulfur. The CVT technique does not include excess sulfur because doing so decreases the transport rate of species whereas pyrite will crystalize in a melt containing excess sulfur which only affects the amount of $\mathrm{FeS}_{2}$ able to dissolve. ${ }^{15,35,36}$

The central hypothesis is that $\mathrm{FeS}_{2}$ grown in Te/S melts will have a low density of $\mathrm{S}$ defects improving photovoltaic properties. Pyrite will dissolve into pure Te as well as Te/S mixtures. Inconclusive evidence suggesting homoepitaxial $\mathrm{FeS}_{2}$ growth has been 
reported. ${ }^{37}$ Single-crystal $\mathrm{FeS}_{2}$ grown in a Te melt were highly resistive compared to samples grown via other methods, ${ }^{38}$ suggesting more pure crystals, however their photovoltaic performance was never investigated.

Conclusive evidence of Volmer-Weber type (island) growth has already been demonstrated from cooling tellurium melts under high sulfur pressure containing dissolved pyrite. Strong evidence of Stranski-Krastnov type (layer then island) homoepitaxial growth of $\mathrm{FeS}_{2}$ has already been obtained with the same procedure. Pyrite has not been grown epitaxially or modified in any way to overcome a $200 \mathrm{mV} V_{\mathrm{ph}}$. The outcome of this research is potentially transformational because it has the possibility of improving the $V_{\mathrm{ph}}$ for pyrite into a level previously unreached, while providing direct evidence for epitaxial growth of pyrite for the first time.

Ideally $\mathrm{FeS}_{2}$ would be grown in molten $\mathrm{S}$ to, as much as possible, limit sulfur vacancy formation. These sulfur vacancies in the surface or bulk are blamed for the low $V_{\text {ph. }}$ Therefore, a high sulfur activity is necessary in a melt when crystallizing pure pyrite. There are no species that consist of only iron and sulfur that have a larger stoichiometric amount of sulfur than $\mathrm{FeS}_{2}$, therefore excess sulfur is not problematic to pyrite formation. Unfortunately, iron does not dissolve in sulfur. Iron and pyrite do both dissolve into liquid tellurium. Tellurium melts at $550{ }^{\circ} \mathrm{C}$ and sulfur is a gas at this temperature, however a high enough sulfur atmosphere can keep the sulfur activity high in the melt. At this point pyrite is the thermodynamically favored species over marcasite $\left(\mathrm{FeS}_{2}\right)$ and troilite $(\mathrm{FeS})$ at high sulfur activities. ${ }^{39} \mathrm{FeS}_{2}$ is also favored over $\mathrm{Fe} / \mathrm{Te}$ species under these condictions. ${ }^{40}$ 
Growing pyrite in Te with sulfur is a reasonable melt to result in a high enough sulfur activity to limit vacancies and defects in the crystals.

Crystal growths utilizing Te/S melts have resulted in the crystallization of pyrite. A two-zone furnace technique has been used in which the resulting crystals have not had high impurities reported. In one case ICP-MS was used to report $155 \mathrm{ppm} \mathrm{Cu}, 20 \mathrm{ppm} \mathrm{Ni}$, $10 \mathrm{ppm} \mathrm{Cr}$ and $3.2 \%$ Te. ${ }^{38}$ These crystals had a high resistivity which indicate that the crystals were of higher purity relative to natural samples and other synthetic pyrite. A similar study utilizing ICP-MS reported $10 \mathrm{ppm} \mathrm{Cr}$ and $20 \mathrm{ppm} \mathrm{Ni}$ in their pyrite. ${ }^{41} \mathrm{~A}$ single furnace method has also been used in an attempt to homoepitaxially grow pyrite onto a natural substrate. The thickness of the growth layer was reported to be $10-50 \mu \mathrm{m}$, however no description of their method was given to obtain these values, and the proposed epitaxial layer was not characterized beyond "optical identification". ${ }^{37}$ None of these crystals have had their photovoltaic performance evaluated and published. This has left a knowledge gap pertaining to the ability of Te/S melt grown pyrite to produce a $V_{\mathrm{ph}}$ and the investigation as to why or why they do not.

The high temperature solution growth can provide a high activity of sulfur throughout the growth process. Moving further this will be the method used for pyrite growth.

\subsection{Experimental}

\subsubsection{Materials}

All chemicals were used as received unless noted otherwise. $\mathrm{Fe}(\mathrm{CO})_{5}(99.5 \%$, Arcos) and sulfur (99.999\%, Alfa Aesar) were received from VWR. Xylenes (98.0\%, 
Arcos), $\mathrm{HCl}$ (aq) (37.4\%, Fisher), and $\mathrm{HNO}_{3}$ (69.3\%, Fisher) were received from Fisher Scientific. Quartz tubing was received from GM Associates. Sulfur (Alfa Aesar 99.999\%), and iron $(99.99 \%)$ were received from VWR. Quartz tubing was received from GM Associates. Single cube pyrite samples were natural and purchased from The Fossil Cartel. Tellurium (Sigma 99.999\%) was received from Sigma Aldrich. KOH (88\%, Fisher) was received from Fisher Scientific. Ethylenediamine (>98\%), ethanethiol $(>98.0 \%)$ were

received from VWR. Sulfur-34 (99.87\%) was from Cambridge isotopes. Concentrated sulfuric acid (ACS grade), ferrocene (98\%), and ferrocenium tetrafluoroborate (technical grade), potassium iodide $(99 \%)$, iodine $(>99.8 \%)$ and cobaltocene $\left(\mathrm{Cp}_{2} \mathrm{Co}, \%\right)$ were purchased from Sigma-Aldrich. Cobaltocenium hexafluorophosphate $\left(\mathrm{Cp}_{2} \mathrm{CoPF}_{6}\right.$, $>95.0 \%(\mathrm{~T})$ ) was purchased from TCI America. Acetonitrile (>99.8\%, anhydrous) was purchased from EMD. Lithium perchlorate (99.99\%, anhydrous) was purchased from VWR. Ferrocene was purified by sublimation. All chemicals except sulfuric acid were stored in a nitrogen-filled glovebox (LABstar MBRAUN, less than $0.5 \mathrm{ppm} \mathrm{O}_{2}$ and $\mathrm{H}_{2} \mathrm{O}$ ).

\subsubsection{Instrumentation}

XRD (X-ray diffraction) patterns were collected with a Rigaku Ultima-IV X-ray diffractometer utilizing $\mathrm{Cu} \mathrm{K} \alpha$ radiation. SEM (scanning electron microscopy) images were captured with a FEI Sirion SEM operating at $5 \mathrm{keV}$ at $5 \mathrm{~mm}$. Raman spectra were collected with a Horiba LabRam HR Evolution microscope using a $532 \mathrm{~nm}$ diode laser at $20 \mathrm{~mW}$ with a 50x objective, $1800 \mathrm{gr} / \mathrm{mm}$ and LabSpec 6 software. TEM samples were prepared with a FEI Strata 237 Dual Beam FIB (focused ion beam) and images captured with a FEI Tecnai TEM/STEM. A Gamry Reference 600 potentiostat was used for I- $V$ 
measurements. SIMS (secondary ion mass spectrometry) depth profiles were obtained with an Ion ToF-SIMS IV (time of flight secondary ion mass spectrometry). A beam of gallium ions was used to remove material form the surface and a beam of cesium ions was used as the primary ion. Profilometry was done with a Veeco Dektak 150 XT profilometer.

\subsubsection{Method of pyrite crystallization with HTSG}

The growth of pyrite was done with a high temperature solution grown in a Te/S melt. This included preparing a substrate to grow onto. A precursor was also synthesized as the source of the pyrite that would form the epilayer. The precursor and melt contents were then combined in the growth chamber followed by the substrate. This was then heated to homogenize the melt. The substrate was then introduced into the melt and then it was all cooled so that the dissolved precursor could crystalize onto the surface of the substrate. The substrate was then removed from the melt and cooled to room temperature. At this point the solidified melt was removed. The details of these experiments are described in more detail in the following text.

\subsubsection{Precursor Growth for HTSG}

The individual building blocks of pyrite must assemble before utilizing the Te/S melt technique to form large single crystals whether they are standalone or part of an epitaxial layer. There are many factors to consider when deciding on a method for making a pyrite precursor. Many forms of $\mathrm{FeS}_{1-2}$ are stable. Two of those forms are both $\mathrm{FeS}_{2}$. One is marcasite and the other our desired product pyrite. The two differ by their crystal structures. Fortunately, pyrite is more thermodynamically favorable across all temperatures ${ }^{39}$ and can be converted from marcasite with thermal annealing in a sulfur 
atmosphere. However, the transformation from $\mathrm{FeS}$ to $\mathrm{FeS}_{2}$ is not as convenient. The diffusion of $\mathrm{S}$ through $\mathrm{FeS}$ is very slow, ${ }^{42}$ therefore it is important to avoid agglomerations of FeS. Two different methods for producing pyrite have been utilized. The first method selected is a synthesis of pyrite from $\mathrm{Fe}(\mathrm{CO})_{5}$ and sulfur in m-xylene. This provides $\mathrm{Fe}$ in the state of a liquid with each atom initially accessible by sulfur. With a high sulfur activity in the solution the chances for $\mathrm{FeS}_{2}$ instead of FeS should be increased. This method has also been used to make precursor pyrite that was then recrystallized with CVT to make photoactive crystals. ${ }^{43}$ The second is the sulfur annealing of iron. This provides a method where only the constituent atoms are involved. Sulfur annealing of iron is a very popular method of synthesizing pyrite. Here elemental pieces of iron are sulfurized but it is also popular to sulfurize iron that has been made into a film by magnetron sputtering ${ }^{44-55}$, flash evaporation $^{56-62}$, and thermal evaporation ${ }^{63-70}$. These films were all polycrystalline.

\subsubsection{Synthesis of pyrite from Iron Pentacarbonyl}

For the purposes of avoiding the formation of iron oxides, the synthesis and the components involved are kept under vacuum or under a nitrogen atmosphere either in a glovebox with $<0.5 \mathrm{ppm} \mathrm{O}_{2}$ and $\mathrm{H}_{2} \mathrm{O}$ or in an atmosphere controlled by a Schlenk line. Prior to the reaction, the glassware used is cleaned with fresh aqua regia to rid of trace contaminants. To obtain an initially high sulfur activity sulfur is dissolved into m-xylene. The system is magnetically stirred as well as heated to encourage dissolution of the sulfur. $\mathrm{Fe}(\mathrm{CO})_{5}$ is then added to the solution. The molar ratio of the sulfur dissolved in m-xylene to the $\mathrm{Fe}(\mathrm{CO})_{5}$ is over 2 which is not problematic as $\mathrm{FeS}_{2}$ has the highest $\mathrm{S}$ to $\mathrm{Fe}$ ratio of the iron sulfur containing compounds. When this happens, the solution goes from slightly 
yellow to black very quickly as the sulfur and iron react. The solution is left alone for 1-2 hours to encourage a complete reaction. The heat applied is then reduced and a needle and syringe are used to remove as much solvent as possible. A vacuum line with a liquid $\mathrm{N}_{2}$ trap are then used to remove and trap the remaining solvent. The resulting powder is then transferred into the nitrogen atmosphere glovebox.

Again, in an oxygen rich environment, iron oxide formation is favorable due to the decomposition of pyrite and the stability of iron oxides. It is imperative to get rid of as much oxygen as possible prior to exposure to high temperatures. To do this a diffusion pump was assembled to evacuate the atmosphere to get rid any residual oxygen as well as to alleviate the increased pressure at high temperatures. The encasing for the annealing needed to be made from something that has a low vapor pressure as well as being strong enough to withstand the vacuum and high-pressure levels at elevated temperatures. Quartz is a material that fits these requirements. It is also relatively easy to manipulate into the desired form and is transparent to light allowing for visual inspection. To obtain a usable vessel, quartz glass was modified with an oxygen/methane torch to the desired geometry to create ampoules for use in sulfur annealing. All the quartz modification was done in house. Quartz tubes were fashioned into a small and a large test tubes. The smaller tube had a small lip at its open side large enough to exceed the inner diameter of the larger tube. The tubes were flame polished and transported into the glovebox where the larger tube was loaded with $\mathrm{FeS}_{\mathrm{x}}$ and $\mathrm{S}$. The smaller test tube was then placed into the larger. Next the larger tube was connected to the diffusion pump where it is then evacuated to $<5 \times 10^{-7}$ torr 
and flame sealed to keep the ampoule atmosphere isolated. The ampoule was then placed in an oven and heated to $600{ }^{\circ} \mathrm{C}$ for $12 \mathrm{~h}$.

\subsubsection{Sulfur annealing iron into pyrite}

Although the diffusion of $\mathrm{S}$ through $\mathrm{Fe}$ is slow, Fe can be annealed in a sulfur atmosphere to convert iron to pyrite. This was used here to determine if there was an advantage of using pyrite made from iron pentacarbonyl.

In a glovebox approximately $0.6 \mathrm{~g}(0.01 \mathrm{~mol})$ of iron pieces were placed into an alumina crucible which was then placed into a flame polished quartz-glass ampoule $14 \mathrm{~mm}$ ID, $150 \mathrm{~mm} 1$ with $(0.04 \mathrm{~mol})$ of sulfur. The ampoule was then connected to a vacuum chamber adapter with a valve closing off the exterior port. The valve was then shut. This was then removed from the glovebox and immediately connected to the vacuum system which was then turned on and the valve opened to the vacuum. Once the pressure reached $5 \times 10^{-7}$ torr the ampoule was flame sealed with an oxygen/methane torch while the end containing the reactants was immersed in an ice-water bath to prevent removal through evaporation and evacuation. The ampoule was then put into an oven which was then heated to $600{ }^{\circ} \mathrm{C}$ and remained at that temperature for $12 \mathrm{~h}$. It then cooled in the oven to room temperature. The ampoule was then transported into the glovebox where the powder could be ground up in a mortar and pestle to ensure complete conversion to pyrite. While grinding after the initial annealing it was noticed that there were still large hard pieces of iron. This indicates that the sulfur had not diffused completely into the iron to fully convert it into pyrite. This process is expected to be slow and a second annealing is not uncommon. The process was repeated with the sulfur annealed iron in place of iron. 


\subsubsection{Substrate for HTSG Preparation}

Epitaxial growth means that the crystal structure of the substrate continues through to the grown layer. If there is a mismatch between the lattice constant of the two layers there will be a strain between them, if this strain is large enough one of the layers will start to break apart. This makes it difficult to grow heteroepitaxial films. This is especially difficult with the current method of growth that takes place between 550 and $650{ }^{\circ} \mathrm{C}$. The thermal expansion coefficient is different between two materials. This can vary the stress between the two layers so that there is less stress during the growth and more at room temperature where the epilayer undergoes stress related damage. In all the published literature there is no evidence of heteroepitaxial growth of pyrite. One group was close to growing epitaxial layers on silicon but there was an oxide layer between the substrate and the over-layer. ${ }^{71}$ In all other cases the layer of crystals grown onto substrates results in a polycrystalline film. ${ }^{3}$ If any of these were analyzed to determine their $n$ - or p-type character

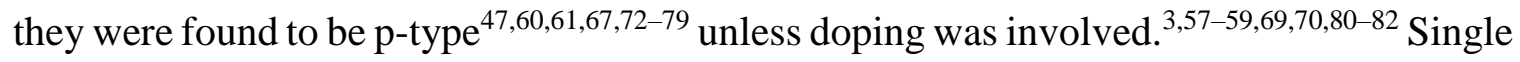
crystals of pyrite have always been shown to be n-type $1,5,21,30,28,83-96$ unless doping was involved $^{35,38,97-100}$. Therefore, the polycrystalline layers must be dominated by the p-type surfaces and not their bulk properties. The goal here is to try and grow bulk films, not polycrystalline layers. This growth is also in a harsh environment. There is a high sulfur activity which can react to produce sulfides which also narrows choices for a substrate. Luckily pyrite forms into the shape of a large cube with each side being a 100 face. This should not undergo stress on the surface as the materials are the same and have the same expansion coefficient and lattice constant. 
The substrate used for growth started off as a large cube of natural pyrite with an edge length of around $1.5 \mathrm{~cm}$. All sides were polished with a sequence of diamond lapping films in decreasing roughness with water as a lubricant leaving a mirror like finish. One of the sides of the cube was then removed with a slow cutting saw so that the thickness of the pyrite was around $2 \mathrm{~mm}$. The unpolished side of the slide was then polished in the same fashion as the cube. The slide was then sonicated in ethanol for fifteen minutes and then rinsed with water and blown dry with nitrogen. A cartoon for this is shown in Figure 2.1.
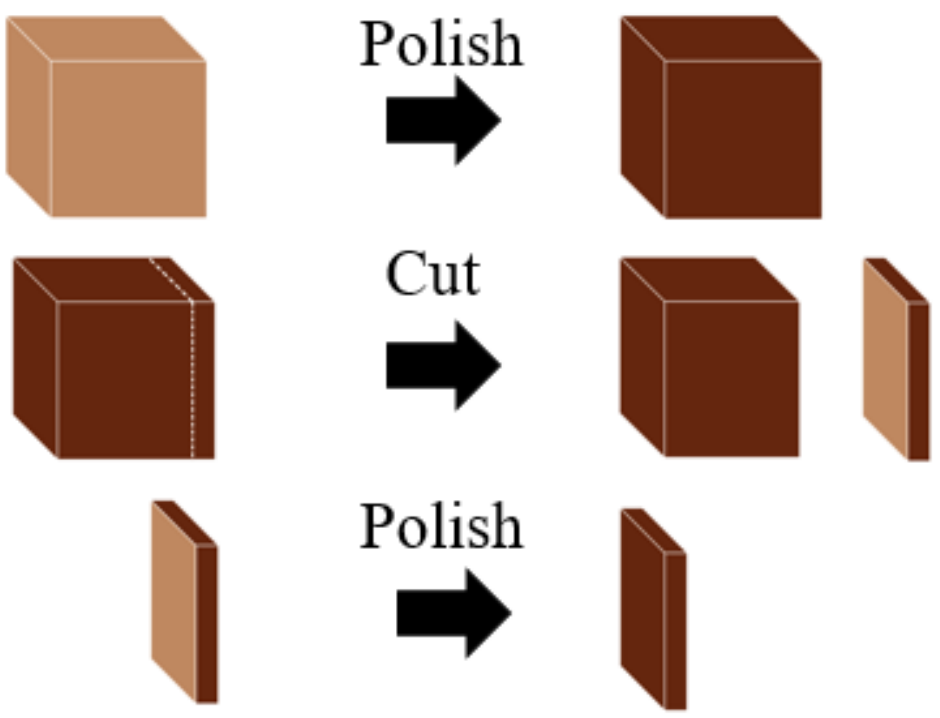

Figure 2.1: Substrate preparation. First the cube is polished on all six sides. Then one of the sides is cut producing a slide of $2 \mathrm{~mm}$ thickness. Then the backside of the slide is polished.

\subsubsection{High Temperature Solution Growth}

\subsubsection{Crystallization}

The homoepitaxial crystallization of the synthetic pyrite onto a natural substrate was accomplished similarly to the sulfur annealing of the pyrite powder. In the glovebox, Te was added to an hourglass-shaped quartz-glass ampoule. The ampoule and its contents 
are illustrated below in Figure 2.2. This purpose of the hour glass is to have an initial separation of the substrate from the solution. $\mathrm{FeS}_{2}$ powder and $\mathrm{S}$ were added. The amount of these three components making up the melt was tested in a similar fashion before addition of the substrate to ensure that all the $\mathrm{FeS}_{2}$ synthetic powder would dissolve. The ampoule was connected to the previously mentioned valve, removed from the glovebox, connected to the vacuum system, evacuated to $>5 \times 10^{-7}$ torr, and flame sealed. The proposed temperature regime for cooling of the melt for crystallization is $650-550{ }^{\circ} \mathrm{C}$. Opening an oven at $650{ }^{\circ} \mathrm{C}$ to manipulate a highly pressurized quartz tube is dangerous. Ideally the ampoule would be able to flip by itself, or in an automated fashion. However, this is not necessary for preliminary studies. The ampoule was placed upright into an in oven spinning apparatus. The oven was then heated to $650{ }^{\circ} \mathrm{C}$ over an hour. At this time the stirring was started, and the oven remained at temp for 6 hours. At that time the oven was opened, and the ampoule was removed from the spinning apparatus and inverted so that the slide was then immersed in the melt. The oven was then closed and cooled to $550{ }^{\circ} \mathrm{C}$ over $5 \mathrm{~h}$. At 550 the oven was opened, and the ampoule inverted so the slide was no longer with the melt. The ampoule was then left to cool to room temperature with the oven off.

This ampoule was flipped upside down so that the melt covered the substrate once homogenization was complete. When the temperature of the oven had cooled to $550{ }^{\circ} \mathrm{C}$ the ampoule was then returned to its original orientation so that the substrate was no longer immersed in the melt. This process is illustrated in Figure 2.2. 


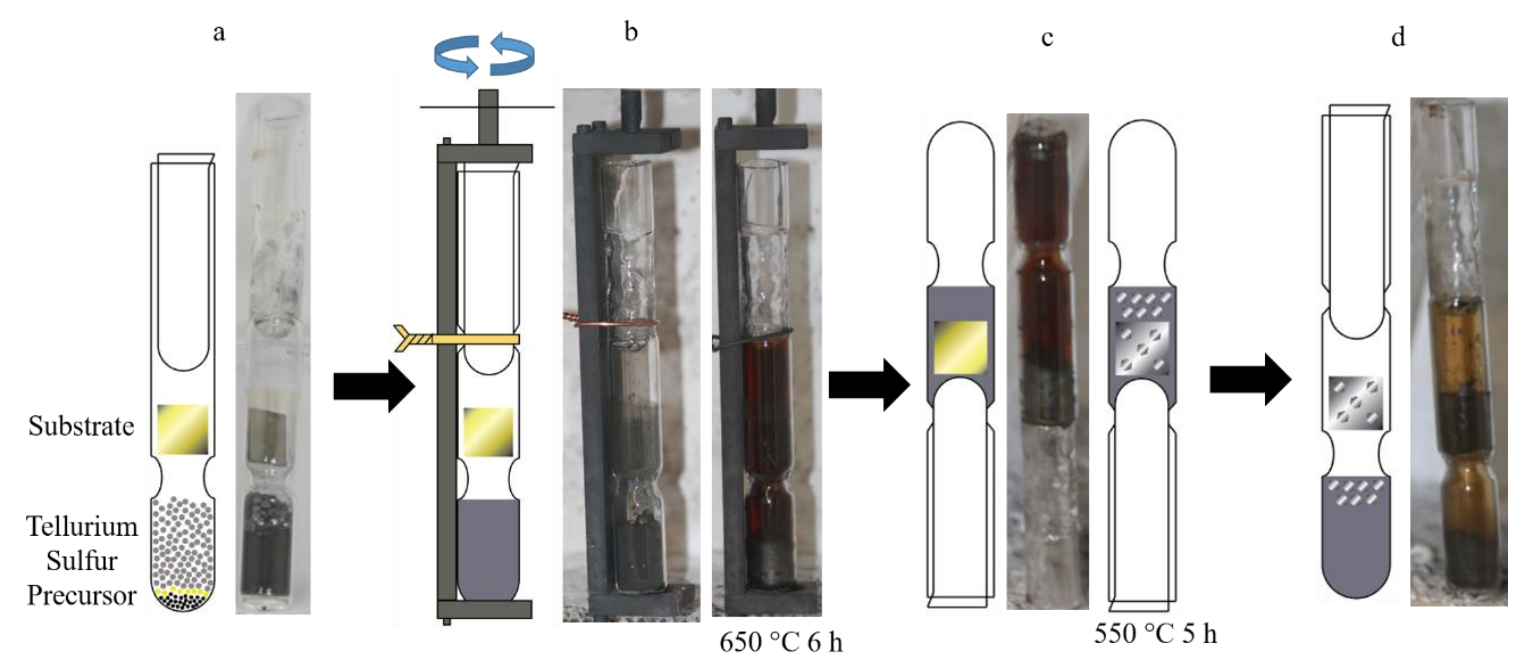

Figure 2.2: Homoeptiaxial layer growth: (a) assembling of the ampoule, tellurium, synthetic pyrite powder, sulfur and a prepared substrate are loaded into a quartz ampoule. The ampoule is then evacuated to $5 \times 10^{-7}$ torr and sealed with a torch. (b) the ampoule is heated to $650{ }^{\circ} \mathrm{C}$ where it remains for 12 hours. (c) the ampoule is then inverted so that the melt is covering the substrate and is then cooled to $550{ }^{\circ} \mathrm{C}$ at a rate of $1 / 3{ }^{\circ} \mathrm{C} / \mathrm{h}$. (e) the ampoule is inverted again to remove the melt from the substrate.

\subsubsection{Removal of solidified Te/S melt}

After the growth there is a layer of solidified melt left on the substrate. Different methods were investigated to remove this melt without also removing pyrite. The first was simply centrifuging the ampoule directly after it had reached $550{ }^{\circ} \mathrm{C}$ so that the melt was still liquid. This was successful in removing large agglomerates of tellurium, but a visible layer remained on the surface. Aqua regia $\left(4: 1:\right.$ concentrated $\mathrm{HCl}$ :concentrated $\mathrm{HNO}_{3}$ by volume) was used to remove the tellurium. The rate at which tellurium was removed was faster than pyrite but not fast enough that the pyrite was left completely intact. This was confirmed by visual inspection of the pyrite surface. The Pourbaix diagram of tellurium shows that oxidizing tellurium is a way of dissolving it without forming hydrogen telluride $\left(\mathrm{H}_{2} \mathrm{Te}\right)$ a toxic gas with an unpleasant smell. An attempt was made to determine the anodic potential to apply the substrate while immersed in an alkali solution to remove the tellurium 
without removing pyrite. The slide was etched in a solution of $4.24 \mathrm{M} \mathrm{KOH}$ with a potential of $+0.2 \mathrm{~V}$ applied to remove the Te layer. This left the surface less damaged than the aqua regia however it was still affected. The most successful method was using a mixture of ethylenediamine and ethanethiol in a ratio of $1: 4$ as described by Webber. ${ }^{101}$ After introducing the slide to the solution the liquid started to darken as tellurium dissolved and the solution became very dark. This was repeated until the solution no longer changed color indicating that all the tellurium was removed from the slide.

\subsubsection{TEM preparation}

Transmission electron microscopy (TEM) allows for a high magnification of a cross section of a material. This technique could provide evidence of the boundary of the substrate and the grown islands. TEM is a technique like SEM. The difference is that instead of analyzing backscattered or secondary electrons that are coming from the incident

surface, electrons that are transmitting through the sample are analyzed. The resolution of this technique allows magnification down to the atomic scale. This requires that the sample thickness to be around $100 \mathrm{~nm}$. To do this a lamella must be created and then removed from the sample. This can be done with a dual beam system. One beam used to remove material, and the other one to deposit a conductive material to make contact of a probe to the lamella. The process for this is illustrated in Figure 2.3. 


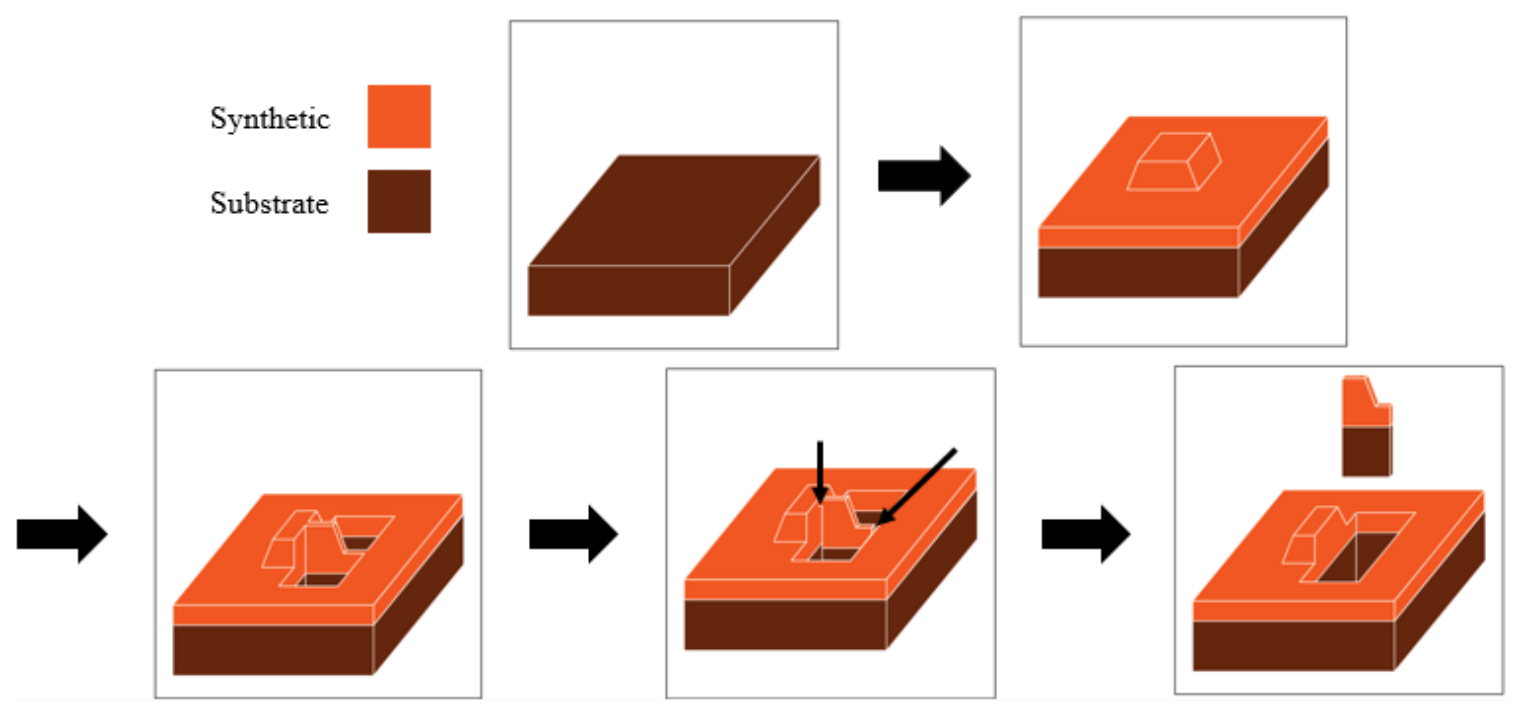

Figure 2.3: Diagram for TEM sample preparation. Brown indicates the substrate (natural pyrite) and orange indicates the synthetic growth layer. First the epilayer is grown onto the natural substrate. Then FIB is used to mill out the area on the edge of one of the growths. Then the lamella is attached to a probe and removed from the sample.

Figure 2.3 depicts the process for producing a lamella out of the pyrite growth. First the HTSG is conducted onto a natural substrate. The natural substrate is shown in brown and the synthetic layer is above it in orange. The cartoon also has one of the island like features as shown on the SEM images. The ion beam of the FIB is used to remove material and create two trenches around the island as shown in the bottom left image of Figure 2.3. Next the lamella is connected to a probe via deposition of platinum. Then a $U$ shape is milled out of the lamella so that it is no longer attached to the sample as seen in the bottom center image of Figure 2.3. The lamella is then lifted vertically out and transferred to a TEM sample holder where it is connected to it by Pt deposition and them the connection to the probe is severed with the ion beam. The sample is then thinned further so it may be examined with TEM. 


\subsubsection{Isotope enriched growth as quantitative evidence for growth}

Qualitative evidence has been provided that the surface of the substrate has changed because of the growth. Without a measurable gravimetric change in the crystals another method had to be used to provide evidence of growth. There are other forms of metal dichalcogenides that have the same crystal structure of pyrite. It may be possible to add these into the growth and then try and determine the thickness of the modified layer. Altering the growth contents in this way may also alter the growth itself as the lattice constant of the other metal dichalcogenides differs from pyrite's. Iron and sulfur each have four isotopes. An isotope is an atom of an element with a different number of neutrons. Each element has its own natural abundance of the isotopes of which it is composed. It may be possible to enrich the relative abundance of one of the isotopes throughout the growth process so that the over-layer is enriched. Then the removal of material from the surface with mass spectrometry can be used to identify the abundance of the different isotopes to determine where the interface of the substrate and the over-layer is. This idea is illustrated in Figure 2.4. 


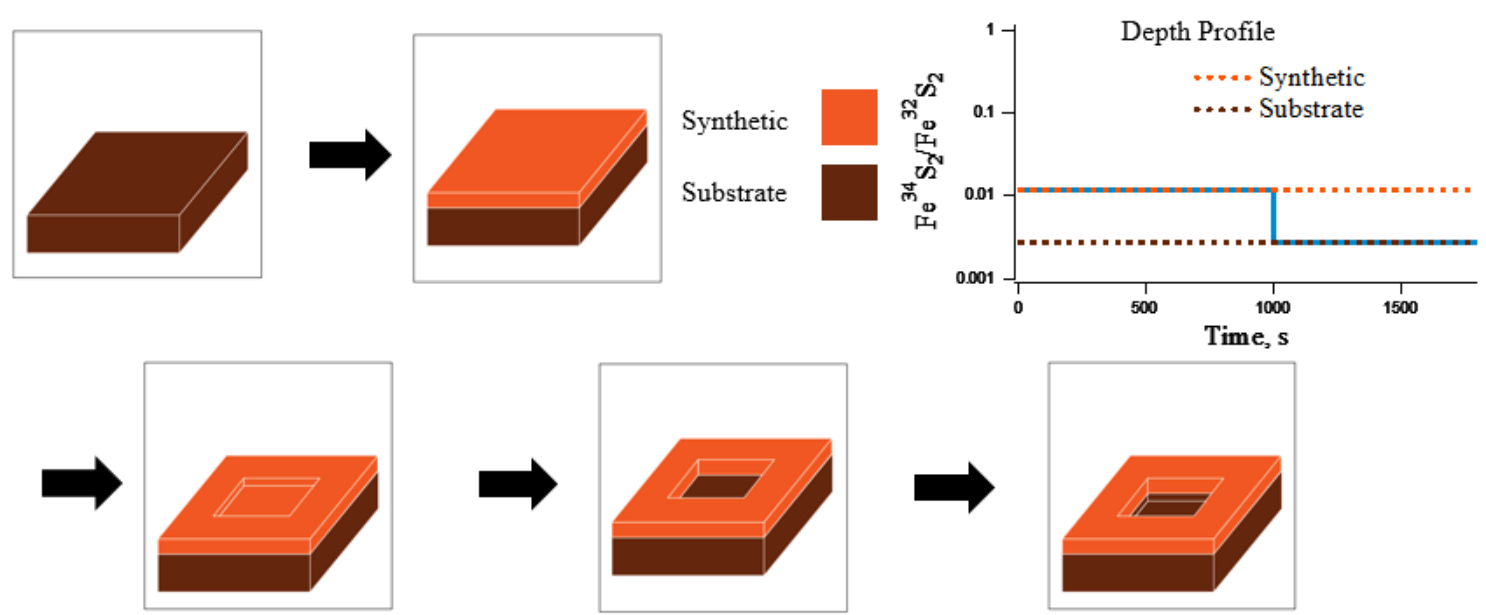

Figure 2.4: Diagram of SIMS experimental design with predicted depth profile outcome. In the diagram brown represents the natural pyrite substrate and orange represents the synthetic epilayer. HTSG is carried out onto a natural slide of pyrite. Then a depth profile is created by milling into the surface and mass analyzing the removed material. Based on the level of doping there should are two predicted ratios of pyrite, one made of natural levels of sulfur and one of pyrite with doped levels of sulfur-34.

In Figure 2.4, a natural slide is prepared for growth, shown in brown. Then a layer is grown on top of it by a HTSG. For the entirety of the precursor synthesis and the growth the normal sulfur has been altered so that there is an enriched amount of sulfur-34. After the growth a depth profile is obtained with secondary ion mass spectrometry (SIMS). This is done by first creating secondary ions on the surface by sputtering them with Ga ions. This results in the ionization of pyrite which can create many different ions that are then collected by the instrument and analyzed. The method of analysis is mass spectrometry. Here a spectrum of the mass to charge ratio is developed with the relative intensity of each provided. The mass to charge ratio is different for different isotopes so two isotopes can be resolved. This process is rastered over an area creating mass spectrum for many spots determined by the area size and the resolution. Then a beam of Cs is used to remove material from the surface and the process is repeated. This produces a tremendous amount of data that can be used to determine elemental information in a three-dimensional area. 
Now that a method of characterization has been decided upon, the signal used for differentiation must be determined. The mass spectrometer used was flooded by the high signal from ionized sulfur and sulfur-sulfur dumbbells. The next most abundant signal was from $\mathrm{FeS}_{2}$ ions. These were chosen for analysis.

Table 2.1: Isotopic abundances of natural sulfur and iron as well as modified abundances of sulfur. $\mathrm{S}_{\mathrm{A}}$ and $\mathrm{Fe}_{\mathrm{A}}$ represent the natural abundances of the isotopes of sulfur and iron. $\mathrm{S}_{\mathrm{MA}}$ represents the modified abundances of the isotopes of sulfur due to the addition of sulfur-34.

\begin{tabular}{|c|c|c|c|c|c|}
\hline \multicolumn{2}{|c|}{ Natural Sulfur } & \multicolumn{2}{|r|}{ Iron } & \multicolumn{2}{|c|}{ Modified Sulfur } \\
\hline Isotope & Abundance $\mathrm{S}_{\mathrm{A}}$ & Isotope & Abundance $\mathrm{Fe}_{\mathrm{A}}$ & Isotope & Abundance $\mathrm{S}_{\mathrm{MA}}$ \\
\hline 32 & 94.93 & 54 & 5.85 & 32 & 89.94 \\
\hline 33 & 0.76 & 56 & 91.75 & 33 & 0.72 \\
\hline 34 & 4.29 & 57 & 2.12 & 34 & 9.33 \\
\hline 36 & 0.02 & 58 & 0.28 & 36 & 0.02 \\
\hline
\end{tabular}

Table 2.1 shows the natural abundances of sulfur and iron as well as a modified amount of sulfur isotopes. It is possible to determine the probability that pyrite $\left(\mathrm{Fe} \mathrm{S}_{2}\right)$ will be made of different isotopes by multiplying the abundances of the individual isotopes as shown below.

$$
F e_{A} \times S_{A} \times S_{A}=F e S_{2 A}
$$

The sum of all the isotope combinations is equal to 1 . This calculation can be redone with the modified abundances shown in Table 2.1. Here extra sulfur-34 has been added to natural sulfur and the abundances recalculated. A table of the results of these calculations can be seen in the appendix. This table shows that there are more than one combination of isotopes that add up to a molecular weight. These possibilities can be summed to come up with a ratio for each molecular weight. Then the ratio of a molecular weight to the highest abundance molecular weight (120) can be determined for both the natural and modified. 
The highest of the ratio of these two numbers are the two signals of interest. In this case it is the molecular weights of 120 and 124 which are represented in figures as $\mathrm{Fe}^{32} \mathrm{~S}_{2}$ and $\mathrm{Fe}^{34} \mathrm{~S}_{2}$. This also gives us our predicted values of the ratios of the signals for 120 and 124 for natural and synthetic.

Another growth was completed by substituting sulfur with a natural abundance for sulfur with a natural abundance combines with sulfur-34 so that the modified abundance was equal to amounts shown on the left in Table 2.1. The precursor synthesis was repeated using iron pentacarbonyl and sulfur. Instead of $1 \mathrm{~g}$ of sulfur, $0.95 \mathrm{~g}$ of sulfur and $0.05 \mathrm{~g}$ of sulfur-34 was used. During the sulfur annealing $0.358 \mathrm{~g}$ of sulfur and $0.022 \mathrm{~g}$ of sulfur-34 were used. The melt of the high temperature solution growth had $0.427 \mathrm{~g}$ of sulfur and $0.024 \mathrm{~g}$ of sulfur-34.

\subsubsection{Electrochemical studies of HTSG/natural pyrite samples}

Electrochemical characterization of photovoltaic performance of pyrite is commonly done by creating an electrical contact in two ways. First involves contacting the pyrite material by making a metal-semiconductor Schottky junction to test it's currentvoltage performance, while the later utilizes an electrochemical cell, in which a liquid electrolyte with a specific redox couple (with defined reduction potential variable by redox couple concentrations as shown in equation 4 is used to contact the pyrite. When there is a junction made between semiconductor and metal, a thermal equilibrium of semiconductor's Fermi level and metal's work function is established. ${ }^{102}$ This is a result of charge migration across the interface that is formed. Studies of such junctions were able to identify a formation of interfacial defects ${ }^{103}$. These defect layers result in so called Fermi 
level pinning that limits the photovoltage of such junctions. Electrochemical methods of contacting that involve liquid solutions of redox couples were shown to not result in defects formation on the interface, hence resulting junctions are "softer". Such approaches were used before for studying $\mathrm{Si}$ and GaAs semiconductors and resulted in demonstration of higher photovoltage values that those obtained for semiconductor/metal junctions. ${ }^{104}$ Since controls of crystallization and bulk stoichiometry of pyrite samples, aimed at $V_{\mathrm{ph}}$ improvement, is of primary focus of this work, it is imperative to avoid limitations of photovoltaic performance of pyrite that could result purely from junction formation. For this, focus was directed on applications of electrochemical pyrite characterizations using redox solutions, as will be discussed below.

\subsubsection{Pyrite electrode design}

Pyrite electrodes are commonly made by first contacting the back of the electrode with $\mathrm{Ga} / \mathrm{In}$ eutectic. ${ }^{1,17,92,105-108}$ Then a tinned copper wire is coiled to a flat surface on one end. The flat surface is then painted with silver paint and contacted to the $\mathrm{Ga} / \mathrm{In}$ eutectic. The wire is then threaded through a tube of borosilicate glass. Then epoxy is applied to seal the connection between the pyrite and the glass tubing. A diagram of this electrode is shown in Figure 2.5. 


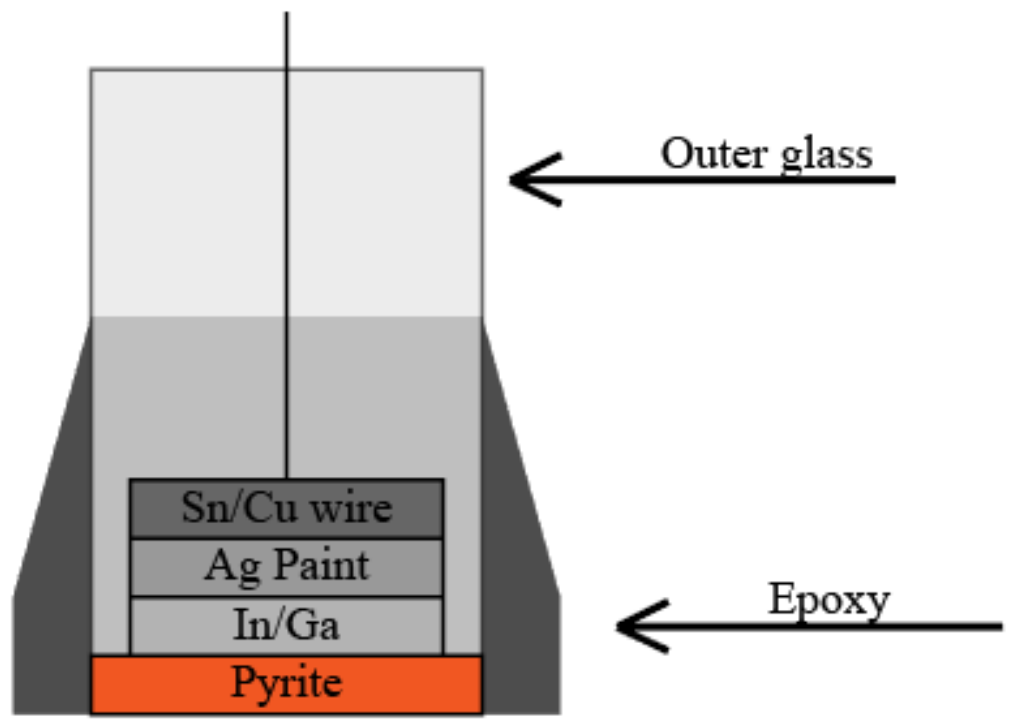

Figure 2.5. Schematic of classic style of pyrite electrode.

This electrode works well for electrochemical experiments with a liquid electrolyte.

The epoxy isolates the surface of the pyrite as the only contact to the solution. However, this design does not allow for a convenient separation of the pyrite sample from the electrode for further characterization with other methods after it has been tested electrochemically. To address this, a specific electrode was designed so that it does not involve permanent seals with epoxy. As seen in Figure 2.6, a circular pyrite sample is mechanically secured between two O-rings that places the studied surface of pyrite in the end of the outer glass tube. 


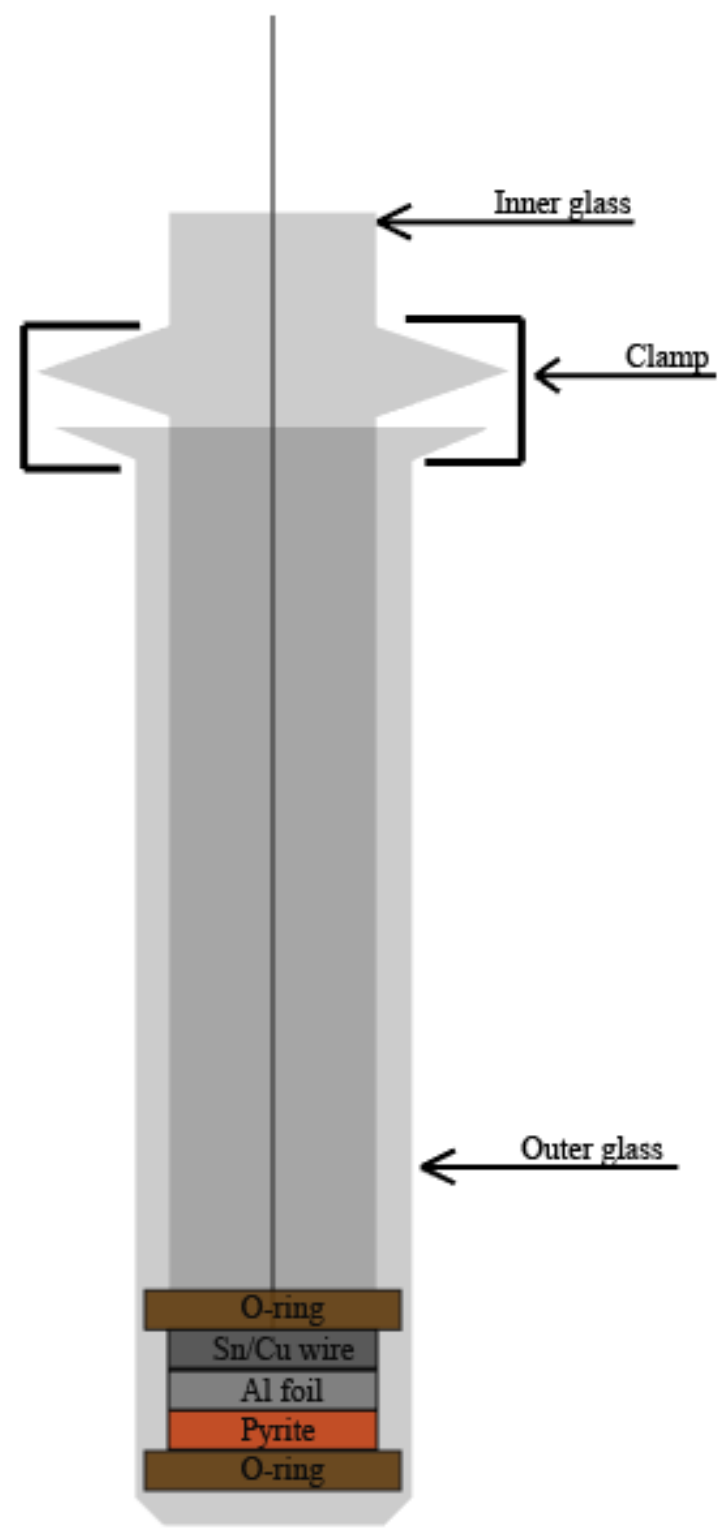

Figure 2.6: Diagram of electrode setup.

An outer glass housing was made of quartz tube that had a smaller opening on one end so that an O-ring could be placed without being forced out of one end. The other end of this tubing was also modified to accommodate for a metallic clamp. On top of the first O-ring the pyrite sample is placed followed by multiple layers of conductive aluminum foil that would be able to mold to the surface of the pyrite so as not to impart stress and damage 
the pyrite sample. Placed on that was tinned copper wire that had been coiled and then soldered together with the tail sticking up out of the top of the glass. On top of that was placed another O-ring. The inner glass housing was made of quartz glass. This was expanded towards the top to meet the clamp. After assembly, the clamp was tightened so that solution would not get into the encasing from the bottom. This allowed for an electrode that could be taken apart for treatment or characterization in between electrochemical characterizations. The ability to assemble the cell inside the glovebox also allows for testing the sulfur annealed pyrite without exposing it to ambient atmosphere.

\subsubsection{Cell design and redox couples}

A custom electrochemical cell was designed and fabricated for photovoltage measurements of pyrite. A schematic of the cell deign is shown in Figure 2.7. The cell includes the counter electrode, a sheet of glass covered with a layer of fluorine doped tin dioxide (FTO). The size of the cell was chosen so that only $2 \mathrm{~mL}$ of electrolyte solution was necessary for measurements. An O-ring was placed between the FTO and the external housing of the cell that was custom made from borosilicate glass tube. Below the FTO counter electrode, another O-ring was placed to reduce the amount of stress applied from the metallic clamp as shown in Figure 2.7. This clamp was used to create a seal between the O-ring and the FTO electrode. The assembled cell was then filled with approximately $2 \mathrm{~mL}$ of solution and a magnetic stir bar was added. The setup was secured above a solar simulator on a ring stand. The previously described electrode containing the pyrite sample was then added into the cell covering the stir bar and being placed as close to the counter electrode (FTO) as possible to limit the solution's resistance. 


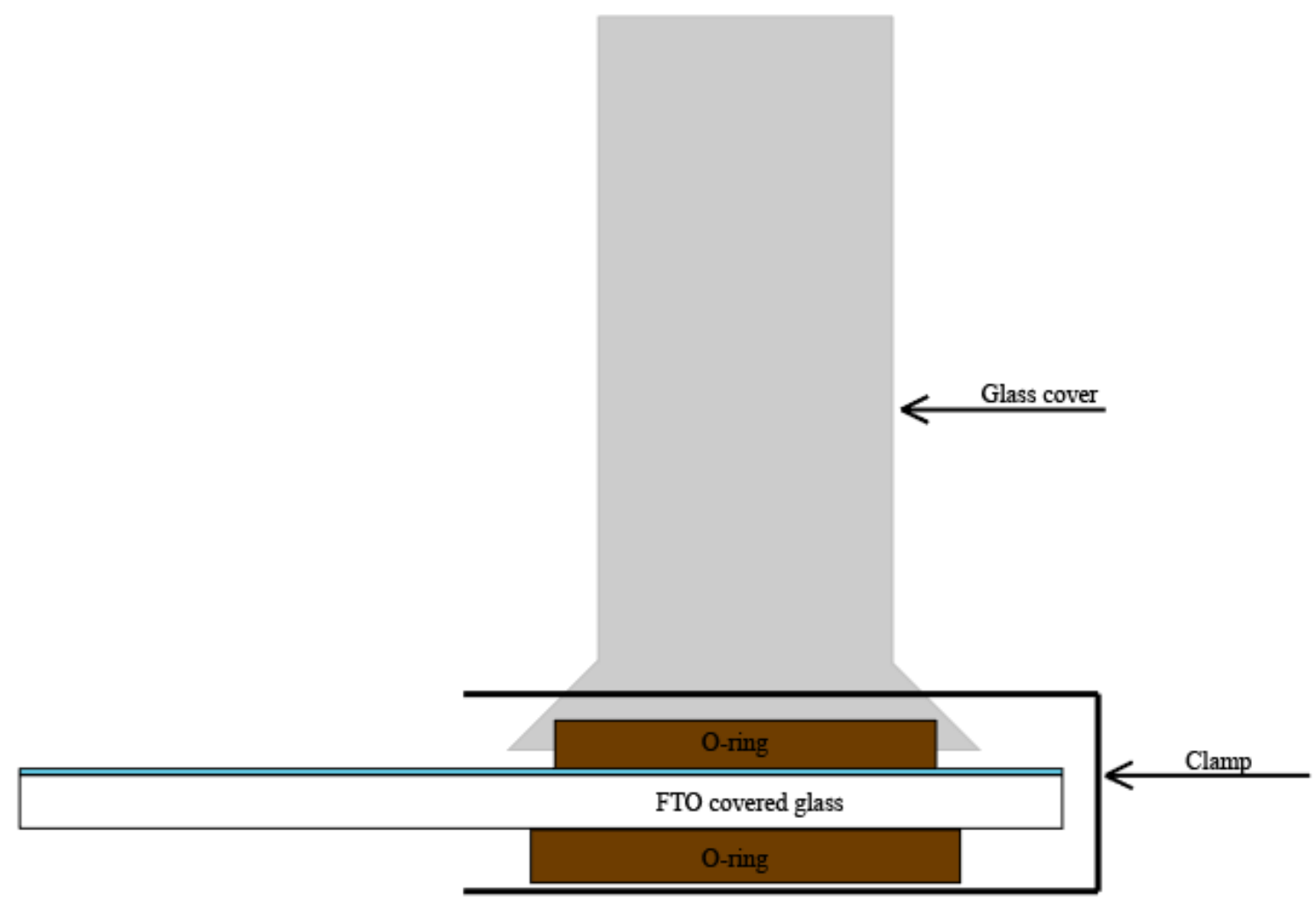

Figure 2.7: Electrochemical cell schematic.

Further described three electrodes were used to study the photovoltaic properties of pyrite in such cells. All electrolytes were prepared in anhydrous acetonitrile. $1 \mathrm{mM}$ ferrocene/1 mM ferrocenium tetrafluoroborate with $100 \mathrm{mM}$ of lithium perchlorate, $1 \mathrm{mM}$ cobaltocene/1 mM cobaltocenium hexafluorophosphate with $100 \mathrm{mM}$ of lithium perchlorate, or $0.5 \mathrm{M}$ Iodide/0.005 $\mathrm{M}$ triiodide. Lithium perchlorate was included in the first two solutions as a supporting electrolyte that increased the conductivity of the solution. This information is reiterated in Table 2.2. Three of the above described cells were manufactured and each filled with one of these electrolytes which allowed for efficient testing of each pyrite sample by moving it from one cell to the other with acetonitrile rinses in between. 
Table 2.2. Electrochemical solutions used to characterize pyrites photovoltaic performance.

\begin{tabular}{|c|c|c|c|c|}
\hline \multicolumn{2}{|c|}{ Redox couple } & $\begin{array}{c}\text { Concentration } \\
{[\mathrm{red}] /[\mathrm{ox}]} \\
(\mathrm{mM})\end{array}$ & $\begin{array}{c}\text { Supporting } \\
\text { electrolyte } \\
\mathrm{LiClO}_{4}(\mathrm{mM})\end{array}$ & $\begin{array}{c}\text { Reduction } \\
\text { potential, } \\
(\mathrm{V} \text { vs. } \\
\text { vacuum })\end{array}$ \\
\hline $\mathrm{I}^{-} / \mathrm{I}_{3}^{-}$ & iodide/triiodide & $0.5 / 0.005$ & 0 & -4.666 \\
\hline $\mathrm{Cp}_{2} \mathrm{Fe}^{-} / \mathrm{Cp}_{2} \mathrm{Fe}^{+}$ & ferrocene/ferrocenium & $1 / 1$ & 100 & -4.982 \\
\hline $\mathrm{Cp}_{2} \mathrm{Co} / \mathrm{Cp}_{2} \mathrm{Co}^{+}$ & cobaltocene/cobaltocenium & $1 / 1$ & 100 & -3.648 \\
\hline
\end{tabular}

\subsubsection{Sulfuric acid etch}

As demonstrated in literature, in most cases, higher photovoltages were obtained for pyrite samples that have been treated with acidic etching of the surface. $^{3,5,15,23,30,34,28,85,95,103,27,109-111}$ The first successful treatment was 30s in concentrated $\mathrm{HF} /$ Acetic $\mathrm{Acid} / \mathrm{HNO}_{3}$ in a ratio of $1: 1: 2 .{ }^{5}$ Another popular method is $0.5 \mathrm{M} \mathrm{H}_{2} \mathrm{SO}_{4}$ with a cathode current passed at a rate of $-15 \mathrm{~mA} / \mathrm{cm}^{2}$. The surface of pyrite was activated when necessary in a similar fashion in $0.5 \mathrm{Mm} \mathrm{H}_{2} \mathrm{SO}_{4}$, but instead of setting the constant current, a constant potential of $-1.1 \mathrm{~V}$ vs solution was set to control the reaction thermodynamically. This potential was held until a total charge of 1.8 Coulombs $/ \mathrm{cm}^{2}$ was passed. The sulfuric acid solution used in this step had been purged with high purity nitrogen gas for 30 minutes before use. Besides the pyrite working electrode the cell included a platinum wire reference electrode and a platinum wire mesh outer electrode. Both electrodes were made by making a seal with the platinum and soft glass. 


\subsection{Results and Discussion}

\subsubsection{Precursor from iron pentacarbonyl}

XRD was used to characterize the precursor powder which had its iron source as iron pentacarbonyl. XRD utilizes a parallel beam of X-rays that interact with a crystalline sample at different angles that diffract the X-rays producing a signal only when the diffracted X-rays are constructively interfering with each other. At this angle there is a face of the crystal. With the angle and wavelength of the incident beam the distance between lattice planes can be determined using Bragg's law. This is how different crystal structures and identified. In a single crystal at a single orientation only one peak is observed. For powder many peaks are observed as the orientation of the crystals is random. If the peak from a face is wide enough the size of the particles can be determined using the Scherrer equation. The grown powder was too large for this, however useful crystal information was obtained from the products. 


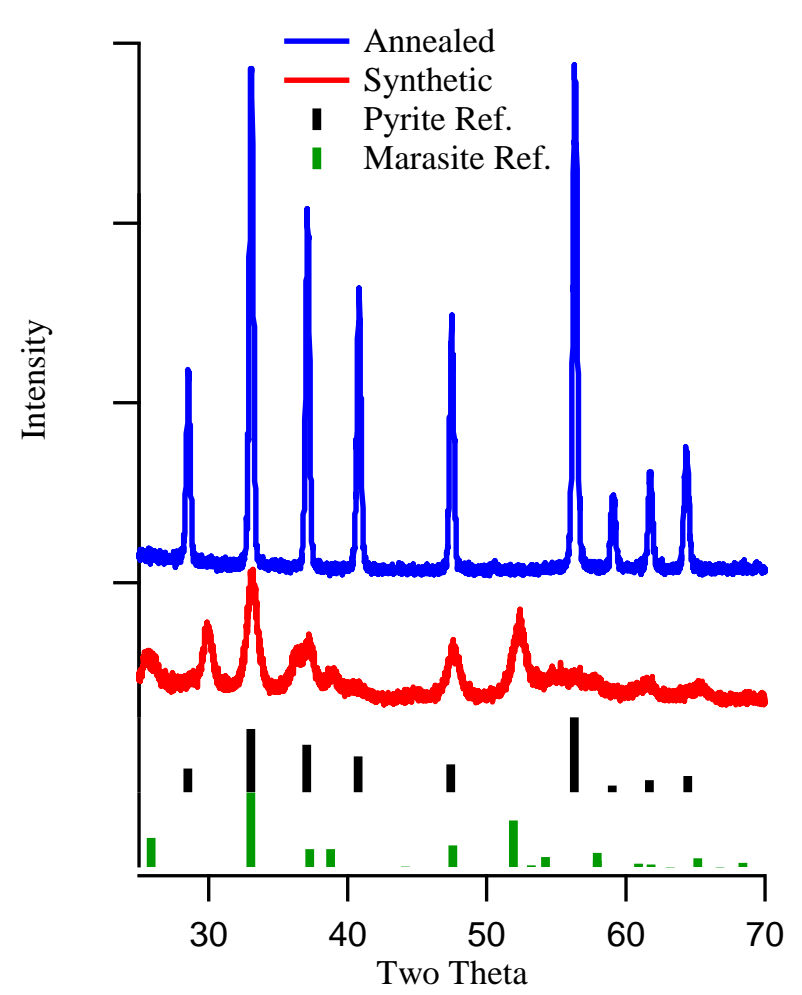

Figure 2.8: XRD pattern of synthetic pyrite directly after solvent removal (red) and after sulfur annealing (blue). References for pyrite (black) and marcasite (green) are included in the figure.

The results of the powder after synthesis showed a mixture of $\mathrm{FeS}_{\mathrm{x}}$ species as seen in Figure 2.8. The peaks here are not very sharp, indicating that the material is not completely crystalline and includes an amorphous not ordered material. Previously it was mentioned that heating causes marcasite to change into pyrite, but that pyrite also degrades into $\mathrm{FeS}$ and $\mathrm{S}$ at elevated temperatures. To negate this sulfur can be added into the atmosphere to create an equilibrium in which the sulfur pressure is high enough to encourage sulfur to combine with the $\mathrm{Fe} \mathrm{S}_{\mathrm{x}}$ as opposed to leaving it. The formation enthalpy for sulfur vacancies in pyrite is very low $(0.1 \mathrm{eV})^{112}$ causing pyrite to decompose at relatively low temperatures. The vapor pressure sulfur over pyrite at $6500^{\circ} \mathrm{C}$ is 172 torr. ${ }^{4,113}$ 
$\mathrm{FeS}_{\mathrm{x}}$ species have been converted to $\mathrm{FeS}_{2}$ in the literature at temperatures as low as 450 ${ }^{\circ} \mathrm{C} .{ }^{114}$

The publication this author was involved in had the sulfur annealing of pyrite at $600{ }^{\circ} \mathrm{C}$ for 12 hours with $50 \mathrm{mg}$ of sulfur added into a sealed tube $14 \mathrm{~mm}$ in diameter and $150 \mathrm{~mm}$ in length creating a sulfur atmosphere much higher than that produced by the pyrite itself. ${ }^{115}$ These parameters more than satisfy the requirements for $\mathrm{FeS}_{\mathrm{x}}$ to $\mathrm{FeS}_{2}$ pyrite transformation.

After sulfur annealing the product the sample was again analyzed with XRD. The results are shown in Figure 2.8. All the peaks in the pattern have small full width half maximums (FWHM) indicating a crystalline structure. The FWHM are small enough that the Scherrer formula cannot be used to calculate the crystal size. All the peaks that are displayed after annealing correlate to pyrite.

To investigate the morphological changes because of the sulfur annealing scanning electron microscopy (SEM) was used to create an image of samples before and after sulfur annealing. SEM works by rastering an image of a sample using a beam of electrons. Electrons undergoing elastic collisions are backscattered. These and secondary electrons from the sample makeup the image and $\mathrm{x}$-rays generated by the sample can be analyzed with specific detectors to obtain elemental information. 


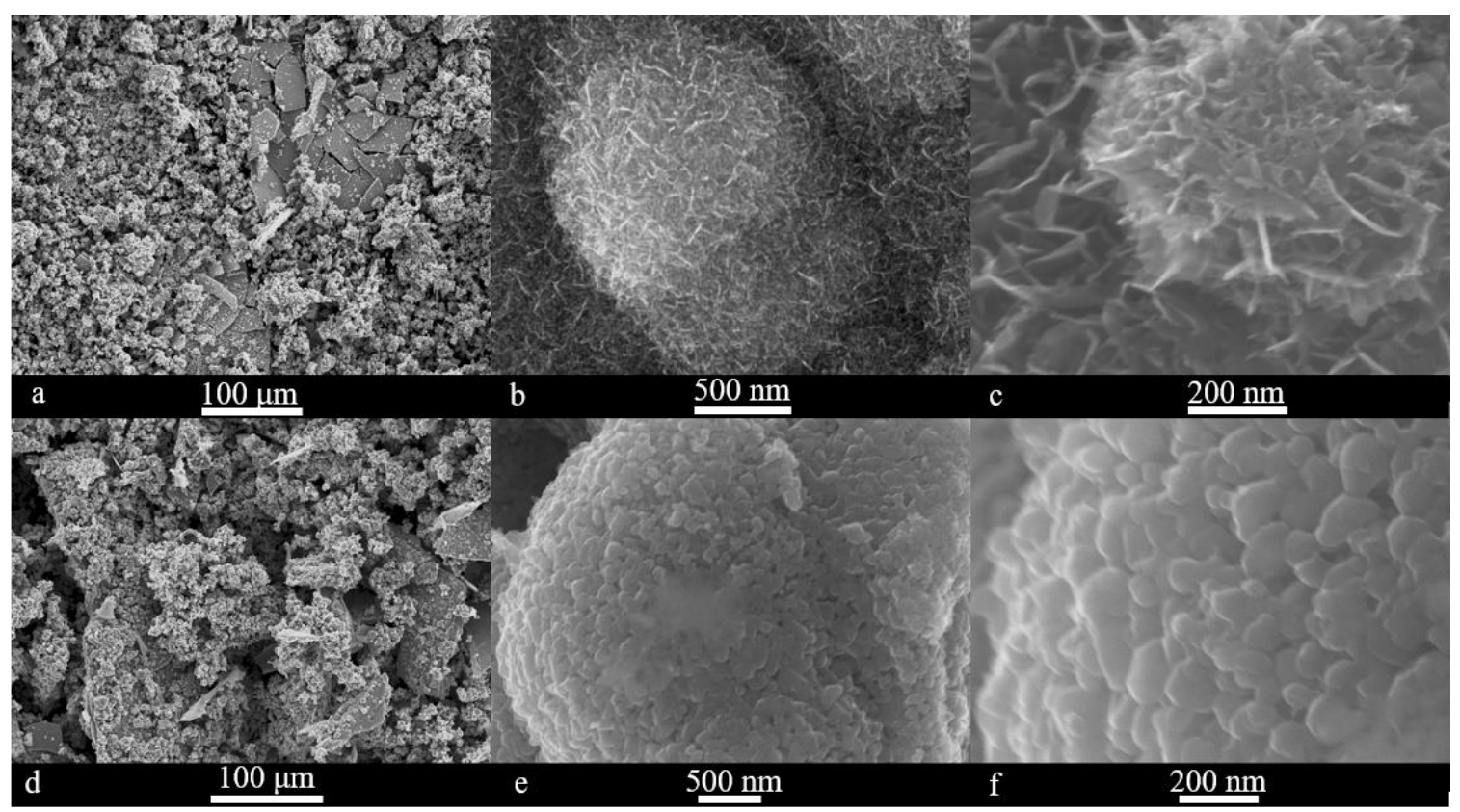

Figure 2.9: Precursor SEM.: SEM images of $\mathrm{FeS}_{\mathrm{x}}$ powder before $(\mathrm{a}, \mathrm{b}, \mathrm{c})$ and after $(\mathrm{d}, \mathrm{e}, \mathrm{f})$ sulfur annealing. An image was taken for each at relatively the same magnification for visual comparison.

Figure 2.9 shows SEM images taken of the synthetic pyrite before and after sulfur annealing. It is hard to tell if there is any difference at the magnification of a and $\mathrm{d}$. However, the differences between $\mathrm{b}$ and $\mathrm{e}$ and $\mathrm{c}$ and $\mathrm{f}$ show that there has been a clear change of morphology on the surface. This is a clear indication that something has changed, the morphology is now small pieces other than long thin structures. They also appear similar to synthetic pyrite in the literature. ${ }^{82}$

There are many different iron sulfides. In fact, there are even two different forms of iron disulfide, pyrite and marcasite. It is important to be able to distinguish the two as the interest is only in pyrite. The band gap of marcasite is 0.34 and would interfere with attempted measurements of only pyrite. Marcasite does have its own crystal structure and is detectable by XRD. In these experiments marcasite was not shown in the XRD pattern, the peaks observed all index as pyrite. It has been acknowledged in the literature that 
Raman spectroscopy is a more sensitive technique to identify the marcasite phase and in some instances XRD did not shown any peaks for pyrite but some were seen in the Raman spectra. ${ }^{116-120}$

Raman spectroscopy uses a monochromatic photon source to excite electrons from vibrational states into virtual energy states. The electrons then fall back into either their original states or states of higher or lower energy. Falling into their original state they produce a photon of the energy of the original laser. This is known as Rayleigh scattering. If the electron falls into a state of energy higher than its original it produces a photon of light of lower energy. The shift of the energy of the photons is known as a Stokes shift scattering. Falling into a state of lower energy it produces a photon of greater energy. The shift of energy of these photons is known as an anti-Stokes shift scattering. The electric field of the photon interacts with the electron cloud of the bond and produces a dipole. The relaxation of this dipole produces the scattered light. Electrons are Raman active if the polarizability of the bond changes with the distance between the two nuclei bonded to each other. These can be predicted by looking at the character table representing the point group. Some of the irreducible representations correlate with transformations of the $\mathrm{x}, \mathrm{y}$ and $\mathrm{z}$ coordinates. If any of the representations correspond to the functions $\mathrm{xy}, \mathrm{xz}, \mathrm{yz}, \mathrm{x}^{2}, \mathrm{y}^{2}$, or $\mathrm{z}^{2}$ or a linear combination of any they are Raman active. The irreducible representations for the point group of pyrite have been known for some time. ${ }^{121}$ There are an $E_{\mathrm{g}}, A_{\mathrm{g}}$, and three $T_{\mathrm{g}}$ modes corresponding to the $\mathrm{S}_{2}$ dumbbell stretch, the $\mathrm{S}_{2}$ dumbbell libration, and three vibrational modes. These result in stokes shifts of $343,379,350,377$, and $430 \mathrm{~cm}^{-1}$. 
These numbers represent the energy shifts in the scattered light. Below is the Raman spectrum for both the synthesized pyrite pre and post annealed in a sulfur atmosphere.
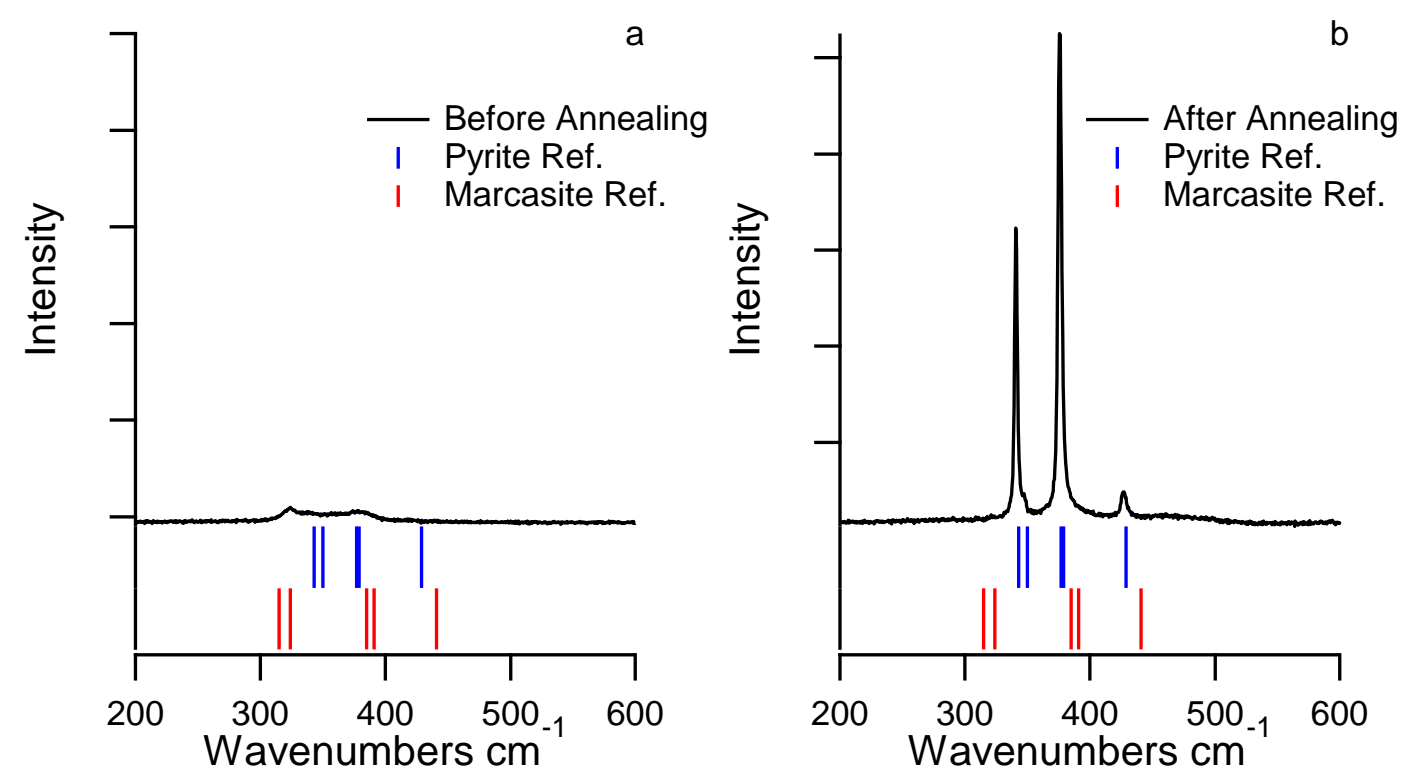

Figure 2.10. Raman of synthetic pyrite before (a) and after (b) sulfur annealing. Pyrite (blue) and marcasite (red) references are included in each.

Figure 2.10 shows the Raman spectra for the synthetic pyrite before and after annealing in a sulfur atmosphere. The pre-annealing does not have any well defines peak. It is difficult based off this information to determine what the sample is, most like amorphous iron sulfides. The post annealing spectrum paints a different picture. The peaks shown here match well with those attributed to pyrite in position as well as in relative intensity. There are no other bands and those expected bands for marcasite are not present either. $^{82,122}$ Therefore the Raman shows that the material is converted to pyrite after annealing. 


\subsubsection{Sulfur annealing iron into pyrite}

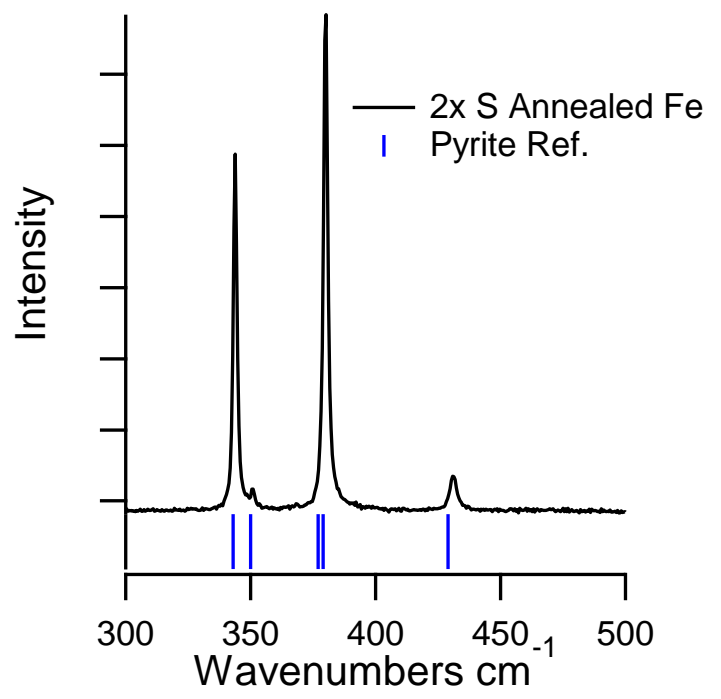

Figure 2.11. Raman spectrum of twice sulfur annealed Fe. A pyrite reference (blue) is included.

Raman spectroscopy was used analyze the sample after the second annealing.

Figure 2.11 shows the Raman spectroscopy of the twice sulfur annealed iron. This is like the previous Raman spectrum of pyrite. The only peaks that show up all correspond to pyrite peaks and the intensity is like the literature. The twice sulfur annealed iron has been fully converted into pyrite.

\subsubsection{Qualitative evidence of HTSG onto natural pyrite}

The simplest way to determine if mass has been deposited of removed from a surface is by looking at the mass difference of the sample before and after growth. This was either too small to measure on the lab scale, or more commonly it was apparent that the surface had also loss significant amounts of the natural substrate during growth so that the final mass was less than the starting. Morphological changes to the surface may also 
provide evidence that there has been a change due to growth. It has been shown in literature that this author was involved in that the surface of the pyrite is very flat after polishing ${ }^{115}$.

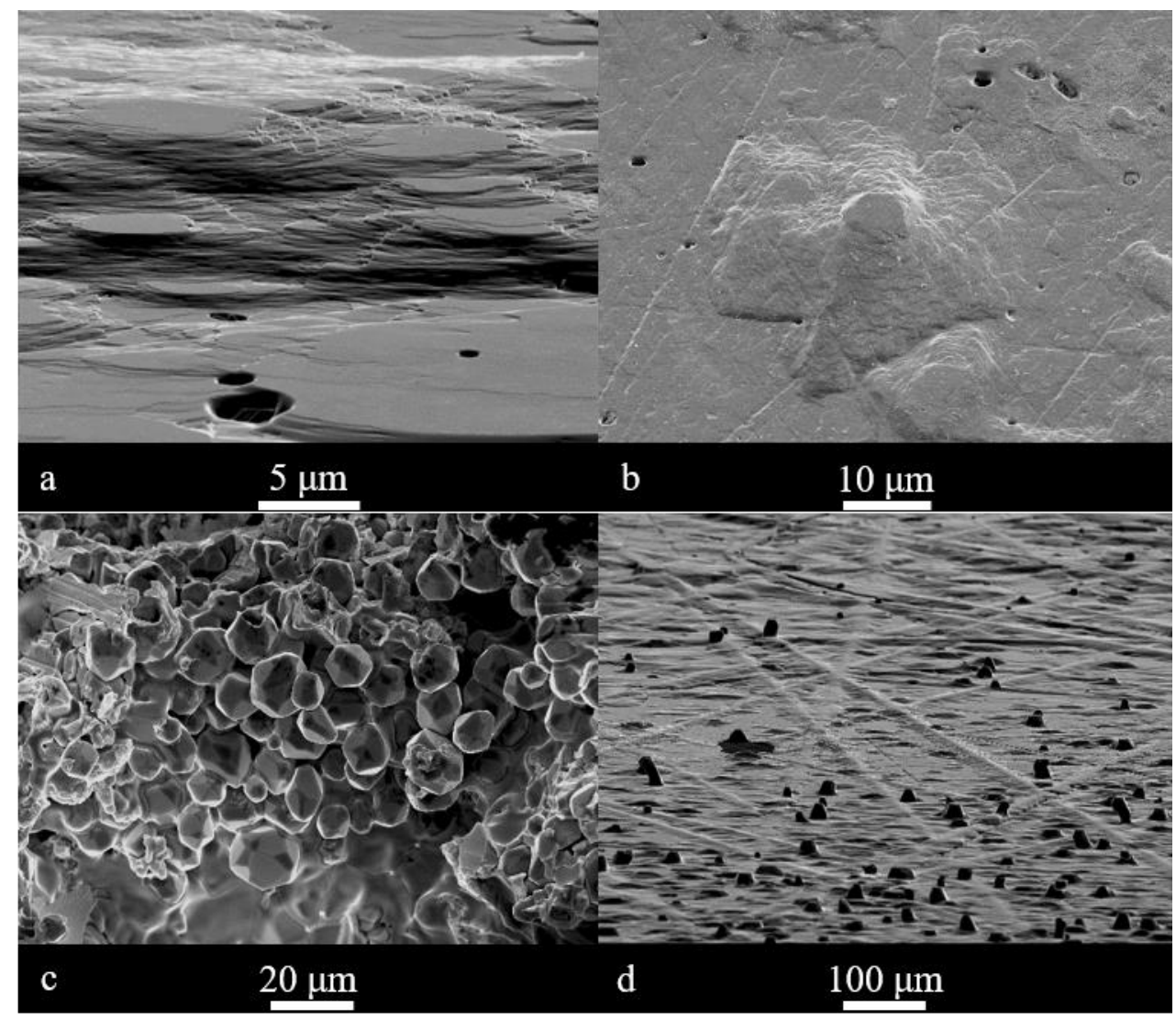

Figure 2.12: SEM images of natural slide having undergone HTSG with the solidified melt removed.

Figure 2.12 shows SEM images of the surface after growth and melt removal.

Figure 2.12.b shows that the surface has changed from being flat with scratch marks. Mesas are now on the surface where it was once flat. These mesas could be the result of the addition of material onto the surface, or the removal of the surface resulting in these structures. Lines through the material are most likely originating from scratches from the polishing process. These have either been grown upon or removed from. In Figure 2.12.d the lines here appear to be coming out of the surface as opposed to into it. This could be 
from a result of the rough scratches providing a favorable center for growth. The shapes from the surface appear to be coming from it. They could have grown from the surface or have fused to the surface. It could be possible that they were once a part of the surface and are a result the removal of their surroundings. Figure 2.12.a is another example of a clear indication that the surface has changed. It is no longer flat and there are many steps. These either indicate growth from the ground up or removal of the material. Figure 2.12.c shows crystals that have been collected in the solidified Te/S melt on the surface of the substrate. These polyhedral do not resemble the shape or the size of the precursor material. They could have been formed from the dissolution and crystallization of the precursor material in the melt but not on the surface of the substrate. There has been a change on the surface due to the growth of the pyrite, however it is not clear if this change was constructive or destructive in nature. Either there are two different growth types observed on the surface. Both Volmer-Weber type (island) and Stranski-Krastnov type (layer then island). Or removal of material has been accomplished in such a way to resemble them. A search for a boundary indicating the interface of the two layers was performed next. 


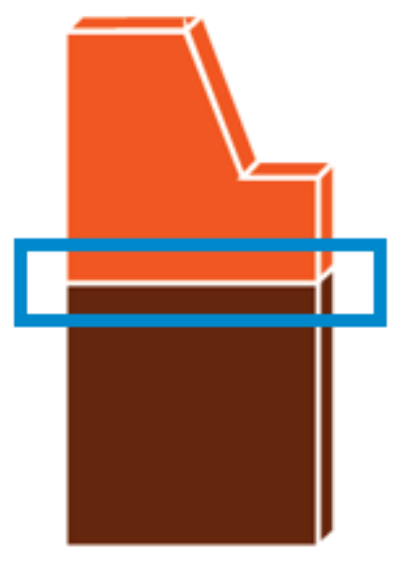

Synthetic

Substrate

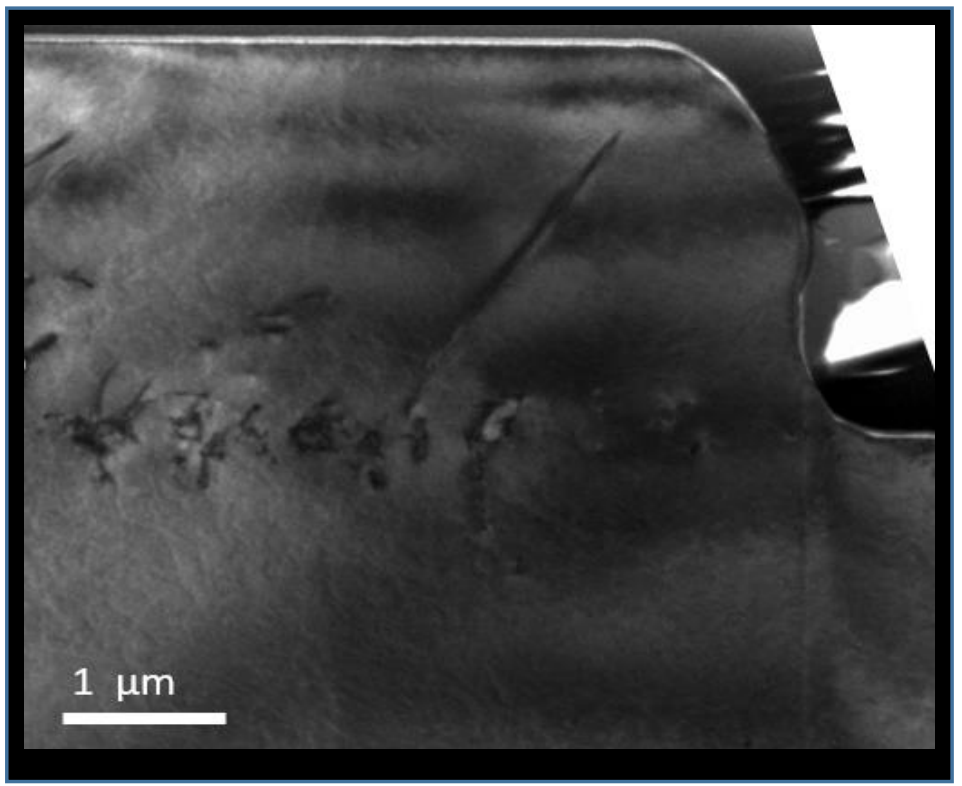

Figure 2.13: TEM sample preparation and a micrograph of the growth mesa. Orange is the synthetic epilayer and brown is the underlying natural substrate.

Figure 2.13 shows a TEM images of the growths on pyrite. The cartoon to the left represents the area of interest to find. The orange is the layer grown on top and the brown is the natural substrate. The blue box is the specific area of interest, a boundary between the two of them. In the TEM image there are defects included in the image at what might be the boundary between the two layers, however this is not strong enough qualitative information to allow us to separate the layers. To determine if there is an atomic difference high resolution TEM (HRTEM) was done to look for the same boundary. 

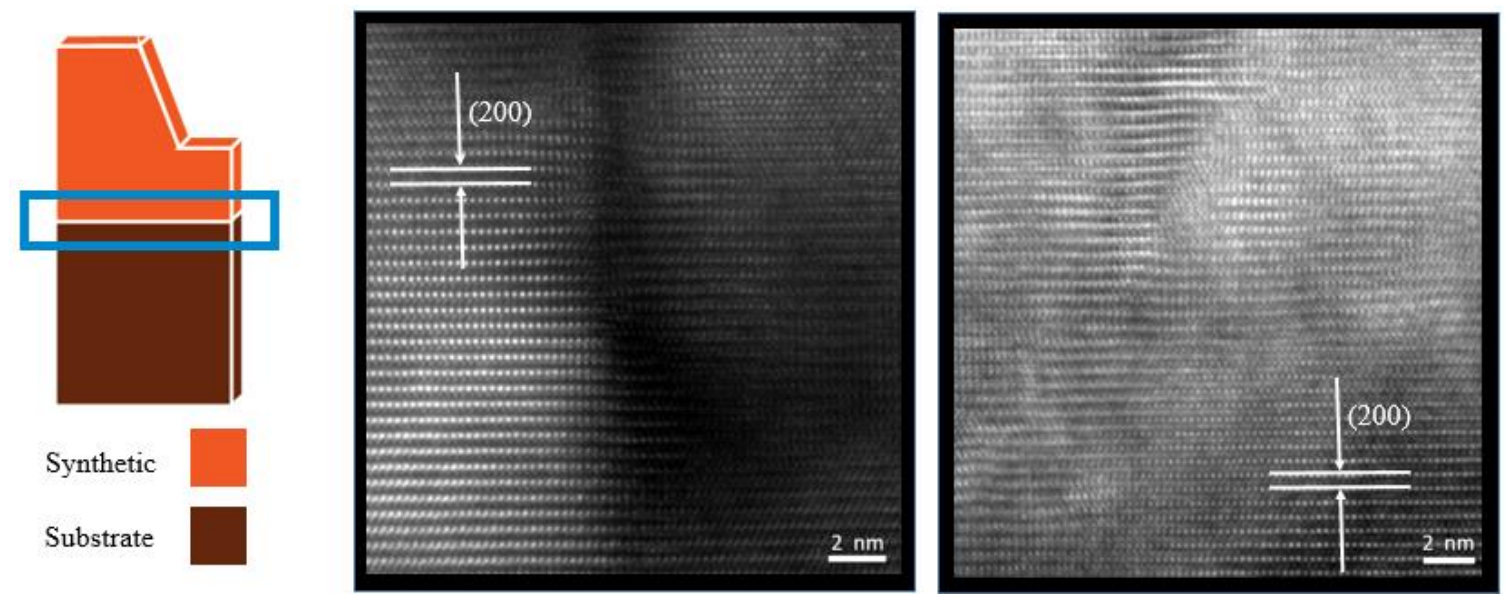

Figure 2.14: HRTEM with orientation identification. In the diagram to the left the orange is the synthetic epilayer and the brown is the underlying natural substrate.

Figure 2.14 shows the cartoon of the area of interest, the boundary between the synthetic layer and natural substrate. There are two images at an atomic scale resolution. These are representative of all the areas searched. There is no clear boundary that can be seen. The lattice appears to continue throughout the images. Both images are marked with the face of the crystal. These have been confirmed with similar images of pyrite in the literature. ${ }^{123-125}$ Without a clear boundary it cannot be concluded that there is a separation between the layers of the natural substrate and the over-layer. However, if indeed it is growth of the surface there was no evidence that the growth was not epitaxial, the lattice appeared to continue from the substrate through the growth layer and the mesa. A more quantitative method is required to conclude that growth has happened.

\subsubsection{Quantitative evidence of growth from isotope enrichment}

A natural slide of pyrite was used as a control to evaluate the accuracy of the calculated values. There is no enrichment of sulfur-34 in this case. 

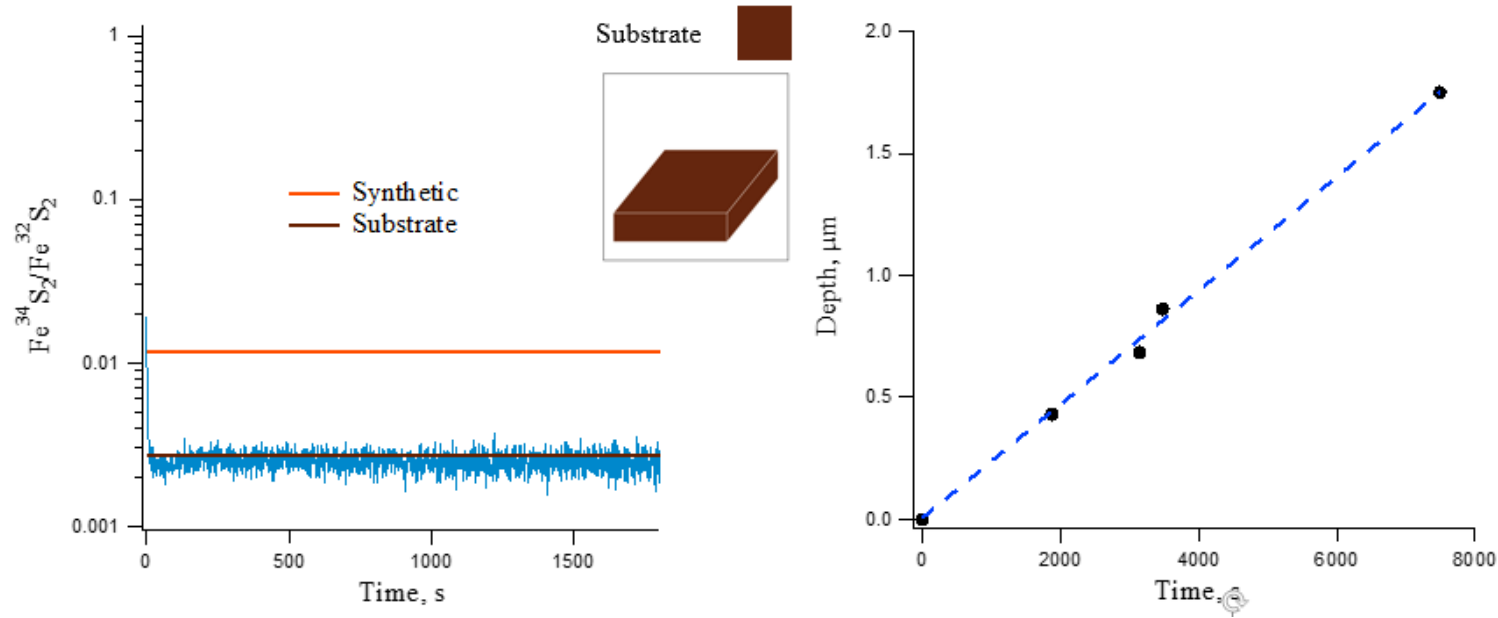

Figure 2.15: Control SIMS depth profile on a polished natural pyrite slide. The orange line is the predicted ratio of isotopes for the synthetic layer. The brown is for the natural layer. b) is a plot of the depth measured by profilometry vs the sputter time.

Figure 2.15 shows the depth profile of the control, a polished slide of natural pyrite.

Two lines are plotted with it. The orange for the calculated value for the ratio of $\mathrm{FeS}_{2}$ comprised of only sulfur-34 to $\mathrm{FeS}_{2}$ comprised of only sulfur 32 for the enriched pyrite and the brown for the natural pyrite. The mass spectrum for the sample is in blue. This matches well with the predicted level for the natural substrate which confirms the calculations.

The depth of material removed could be determined with profilometry. Profilometry is a technique used to obtain a profile for the surface of a material. This can be used to measure the difference in the height of the crater that was formed because of sputtering material and the height of the original surface lateral to the crater. From all the depth profiles the time and depth correlated, and a plot was made to convert the time to depth. The plot of this is shown in Figure 2.15. The remaining profiles will all have depth on the $\mathrm{x}$ axis. 


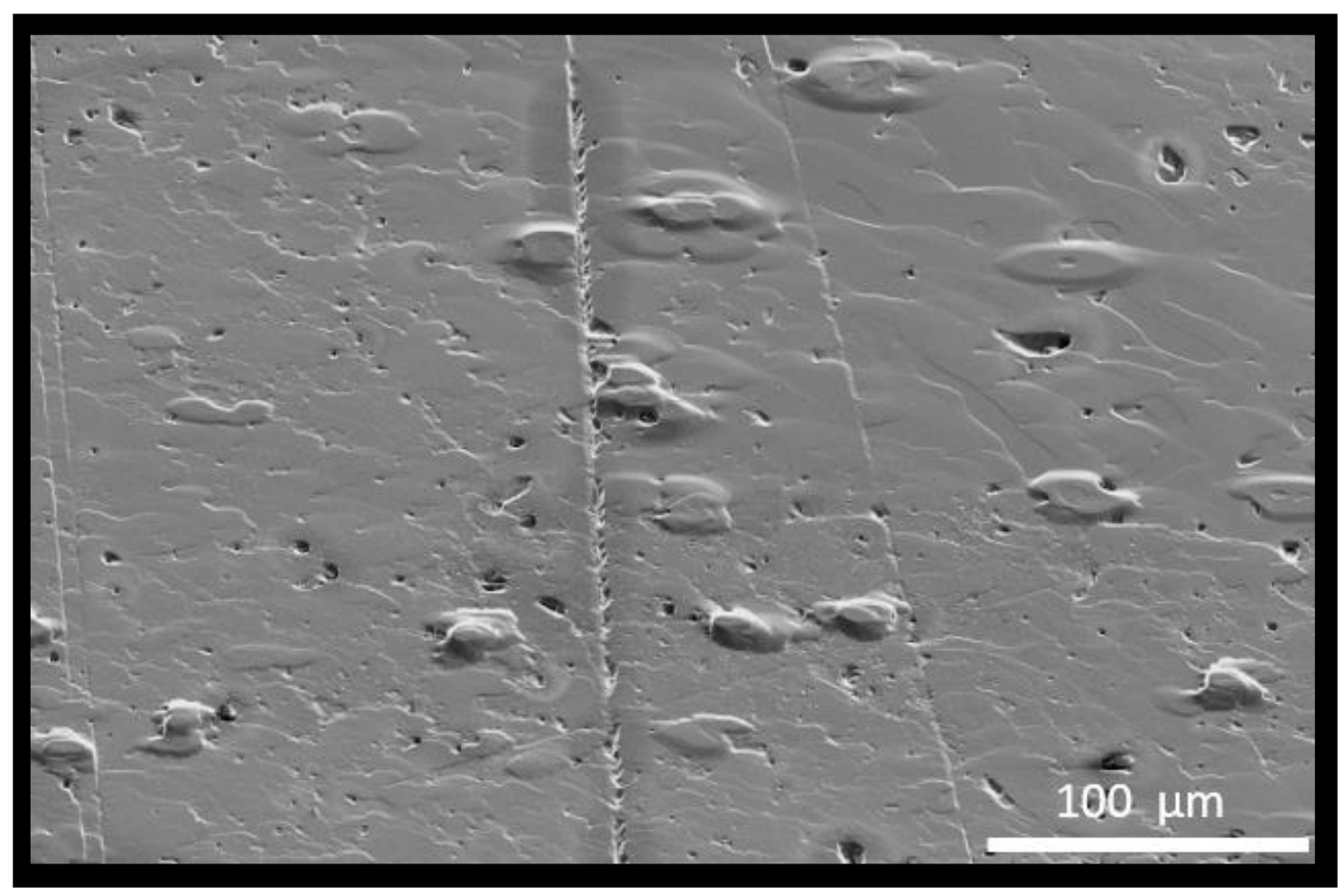

Figure 2.16: SEM image of the area where the SIMS was performed.

Figure 2.16 shows an SEM image of the area to be examined with SIMS. This area was selected because it displayed the different features of possible flat layer growth and the inclusion of mesas. 


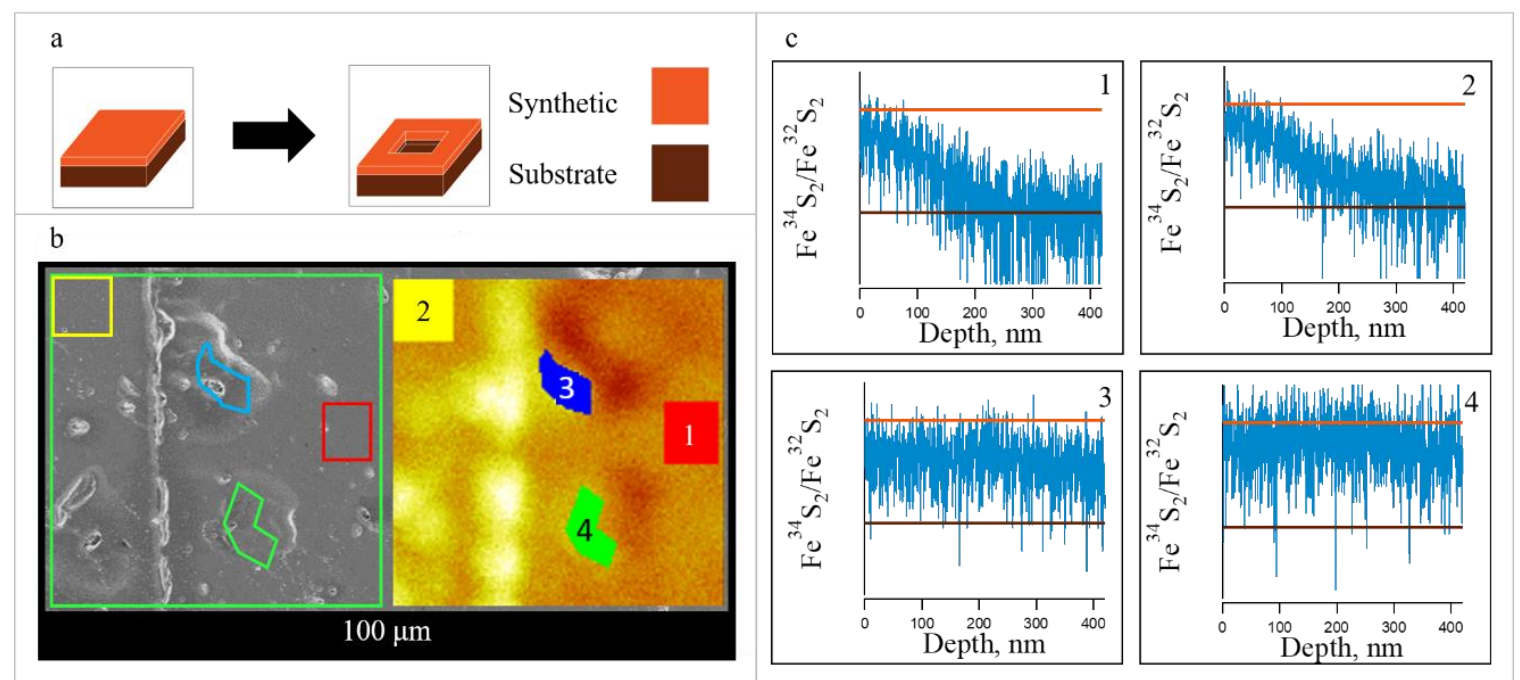

Figure 2.17: a) diagram of the profiling process, here the brown represents the natural substrate and orange represents the synthetic epilayer. b) the area that was profiled with four different regions of interest selected. c) the corresponding depth profiles of each of the four spots including predicted ratios in orange for natural and brown for synthetic.

Areas one and two as shown in Figure 2.17, are both on flat regions of the image. These should start close to the calculated levels for the synthetic material and then drop to levels closer to the natural substrate. This is what is seen in both of their depth profiles. They start at a higher level close to the calculated synthetic level and then fall to a level close to the natural substrate's level. This is not the sharp drop off that was predicted. This may arise from an uneven layer grown on the surface so that while removing material from the surface there is a point where both material from the synthetic layer and the substrate are being removed and sent to the mass spectrometer so that the signal changes steadily and not abruptly. This is illustrated in Figure 2.17. These each show the layer on the surface to be around $100 \mathrm{~nm}$. 


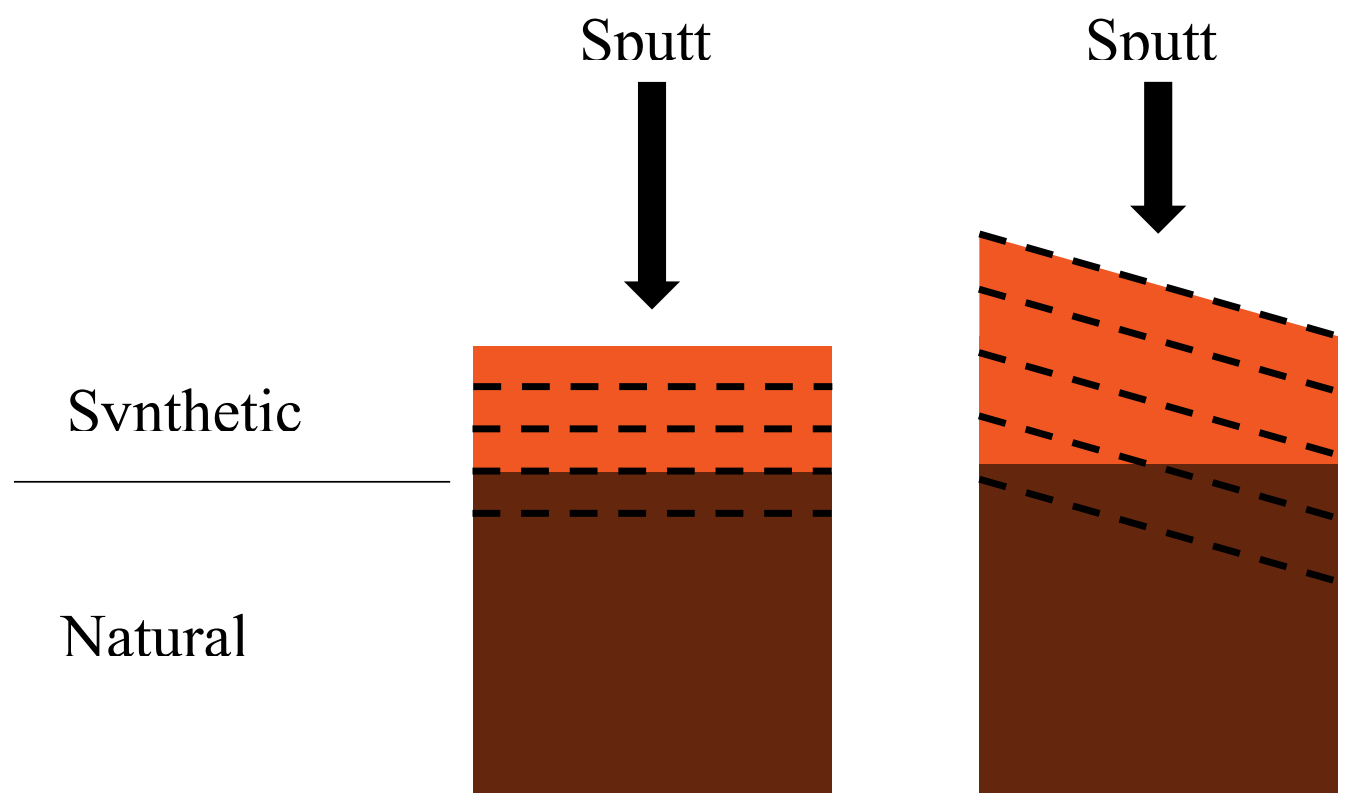

Figure 2.18. Sputtering of two different surfaces. One with a flat layer grown on the surface to the left. On the right an uneven surface growth. Dotted lines represent new surface boundaries after material removal.

Figure 2.18 shows two surfaces. One with a parallel layer grown on the surface and the other with an uneven layer on the surface. For sputtering the layer on the left there is a clear drop between the two layers and a sharp drop would be seen in the signal. While sputtering the layer on the right there would be a region where sputtering would remove layers from both the synthetic and natural layers. This is an explanation for the decrease in signal between the synthetic and natural depth profiles of area one and two.

Areas three and four should both start off and stay at levels near the calculated values for the modified abundances. This is indeed what happens in both of their spectra. There is a flat line that is close to the modified abundance calculation.

Both agree that the new morphology on the surface of the pyrite after growth is a result of material being deposited on the surface not removed. The only other argument 
would be that sulfur-34 diffused into the material and none had grown. Using the experimentally derived diffusion coefficient from Watson ${ }^{126}$ the depth that sulfur-34 would diffuse into pyrite from exposure at a temperature of $650{ }^{\circ} \mathrm{C}$ for 12 hours would only be about $8 \mathrm{~nm}$. This is much smaller than the depths seen here. That suggests that this is indeed growth and not simply the diffusion of sulfur into the surface of the pyrite.

\subsubsection{Photovoltage measurements of HTSG onto natural pyrite}

Table 2.3: Photovoltage measurements for HTSG onto a natural pyrite crystal.

\begin{tabular}{|c|c|c|c|c|}
\hline \multirow{3}{*}{ Sample } & \multirow{3}{*}{ Preparation } & \multicolumn{3}{|c|}{$V_{\mathrm{ph}}$ for pyrite/redox couple junction $(\mathrm{mV})$} \\
\cline { 3 - 5 } & & $\mathrm{Cp}_{2} \mathrm{Fe} / \mathrm{Cp}_{2} \mathrm{Fe}^{+}$ & $\mathrm{Cp}_{2} \mathrm{Co} / \mathrm{Cp}_{2} \mathrm{Co}^{+}$ & $\mathrm{I}^{-} / \mathrm{I}_{3}{ }^{-}$ \\
\cline { 3 - 5 } & & $1 \mathrm{sun}$ & $1 \mathrm{sun}$ & 1 sun \\
\hline \multirow{2}{*}{$\mathrm{HTSG}$ on NAT } & as is & -7 & 0 & -9 \\
\cline { 2 - 5 } & $\mathrm{H}_{2} \mathrm{SO}_{4}$ etched & -7 & -1 & -6 \\
\hline
\end{tabular}

Table 2.3 shows the $V_{\text {ph }}$ values for the HTSG pyrite grown onto natural pyrite substrate both after growth and after etching as a function of contacting electrolyte. Data was collected under 1 sun illumination intensity $\left(100 \mathrm{~mW} / \mathrm{cm}^{2}\right)$. There is not much change before and after etching of the pyrite and the photovoltage is low for all three electrolyte solutions. This indicates the presence of defect states within the bulk or surface of pyrite. Such bulk defects can serve as recombination centers for photogenerated charge carriers (electron - hole pair) or defects on the surface can result in the phenomenon known as pinning of the Fermi level. During the Fermi level pinning, photovoltage produced by the pyrite/redox junction is invariant of the changes in reduction potential of the contacting solution. 


\subsection{Conclusions}

Synthetic iron sulfides or iron were successfully converted into pyrite through a high sulfur activity annealing step. Qualitative evidence of a homoepitaxial growth of synthetic pyrite onto natural pyrite along with quantitative evidence of the thickness of the layer were successfully demonstrated and described in this chapter. It is concluded that the morphological changes observed on the pyrite were a result of crystal growth. However, the resulting growth layers, when grown on the natural pyrite sample, did not produce a notable photovoltage improvement even after application of commonly used surface activating acid etching treatments. Electrochemical data was consistent with presence of either or both bulk defects that serve as recombination layers, or presence of surface defects that result in Fermi level pinning. This was concluded as $V_{\mathrm{ph}}$ values only changed insignificantly with change in reduction potential of the three redox solutions being greater than $1 \mathrm{~V}$ and spanning across commonly reported values of $\mathrm{E}_{\mathrm{vb}}$ and $\mathrm{E}_{\mathrm{cb}}$ of pyrite. 


\section{CVT growth of pyrite as a replacement substrate for HTSG to increase $V_{\mathrm{ph}}$}

\subsection{Introduction}

The thickness of the layer on the surface may be on the order of $100 \mathrm{~nm}$. Natural pyrite was not made in a controlled environment. It is known that here are many elemental defects in natural pyrite. $4,38,83,86,88,127$ These defects may have diffused into the synthetic layer on the surface during growth and created mid-gap states inhibiting the $V_{\mathrm{ph}}$. It is important to use a repeatable substrate for the HTSG and one with defects that do not compromise the structure of the substrate during growth.

\subsection{Experimental}

\subsubsection{Materials}

All chemicals were used as received. Single cube pyrite samples were natural and purchased from The Fossil Cartel. Tellurium (Sigma 99.999\%) was received from Sigma Aldrich. Sulfur (Alfa Aesar 99.999\%), and iron (99.99\%) were received from VWR. Iron bromide ( $\left.\mathrm{FeBr}_{2} 99.995 \%\right)$ was received from Fisher Scientific. Quartz tubing was received from GM Associates. Ethylenediamine (>98\%), ethanethiol (>98.0\%) were received from VWR. Concentrated sulfuric acid (ACS grade), ferrocene (98\%), and ferrocenium tetrafluoroborate (technical grade), potassium iodide (99\%), iodine $(>99.8 \%)$ and cobaltocene $\left(\mathrm{Cp}_{2} \mathrm{Co}, \%\right)$ were purchased from Sigma-Aldrich. Cobaltocenium hexafluorophosphate $\left(\mathrm{Cp}_{2} \mathrm{CoPF}_{6},>95.0 \%(\mathrm{~T})\right)$ was purchased from TCI America. Acetonitrile (>99.8\%, anhydrous) was purchased from EMD. Lithium perchlorate (99.99\%, anhydrous) was purchased from VWR. Ferrocene was purified by sublimation. 
All chemicals except sulfuric acid were stored in a nitrogen-filled glovebox (LABstar MBRAUN, less than 0.5 ppm $\mathrm{O}_{2}$ and $\mathrm{H}_{2} \mathrm{O}$ ).

\subsubsection{Instrumentation}

XRD patterns were collected with a Rigaku Ultima-IV X-ray diffractometer utilizing $\mathrm{Cu} \mathrm{K} \alpha$ radiation. SEM images were captured with a FEI Sirion SEM operating at $5 \mathrm{keV}$ at a distance of $5 \mathrm{~mm}$. Raman spectra were collected with a Horiba LabRam HR Evolution microscope using a $532 \mathrm{~nm}$ diode laser at $20 \mathrm{~mW}$ with a $50 \mathrm{x}$ objective, 1800 $\mathrm{gr} / \mathrm{mm}$ and LabSpec 6 software. A Gamry Reference 600 potentiostat was used for I- $V$ measurements.

\subsubsection{CVT crystal growth}

Chemical vapor transport is a technique were a polycrystalline source at a higher temperature is transported by a halogen containing transport agent to a region of cooler temperature where it crystalizes into single crystals. This has been very popular for pyrite and most of the published data with $V_{\mathrm{ph}}$ measurements have been done on CVT samples. ${ }^{3,5,7,15,19,21,23,30,34,28,35,38,41,43,84,85,87,90-92,96-100,103,27,110,123,128-141}$ Unfortunately the halide transport agent is included within the crystal. ${ }^{35,92}$ Pyrite also decomposes at $300{ }^{\circ} \mathrm{C}$ into iron sulfide and sulfur gas. ${ }^{4}$ The hot and cool ends of pyrite CVT are around 650 and $550{ }^{\circ} \mathrm{C}$. It is reasonable to believe that there are sulfur vacancies in the CVT pyrite. Adding sulfur to the growth greatly reduced the transport rate ${ }^{35}$ These are drawbacks for the material. However CVT crystals exhibit excellent crystallinity which make them good substrates for homoepitaxial growth. ${ }^{123}$ 
For this CVT growth a two-temperature zone tube furnace is not available. Therefore, two zones were created by treating the center of a tube furnace as one temperature zone and the outermost area of the tube furnace as the other area. Synthetic pyrite was used make single crystals in a CVT growth to test this experimental setup

\subsubsection{CVT growth of an over-layer onto natural pyrite}

In the literature the CVT growth is used to produce single crystals in almost all cases. There is one that was used to produce a homoepitaxial film of about $0.4 \mu \mathrm{m} .{ }^{137}$ The idea to replace a substrate is to perform a homoeptiaxial CVT growth onto a natural substrate. The next step to remove the natural substrate from the CVT crystal and then use the CVT crystal as the growth substrate for the HTSG. Figure 3.1 illustrates this idea. 


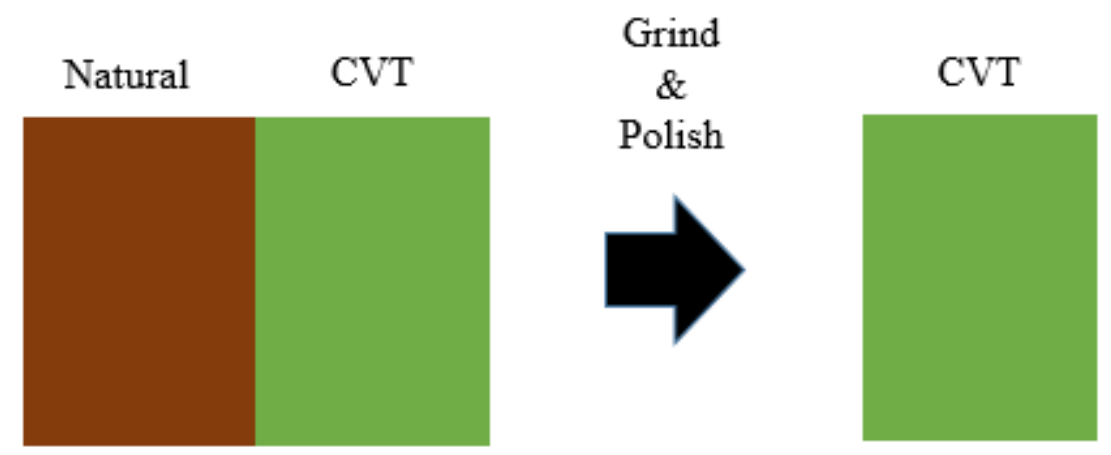

Figure 3.1. Schematic for CVT growth onto natural pyrite and then remove of natural pyrite to leave only the CVT pyrite for use in the HTSG.

Cooler

Seed crystal

Growth

Hot Source
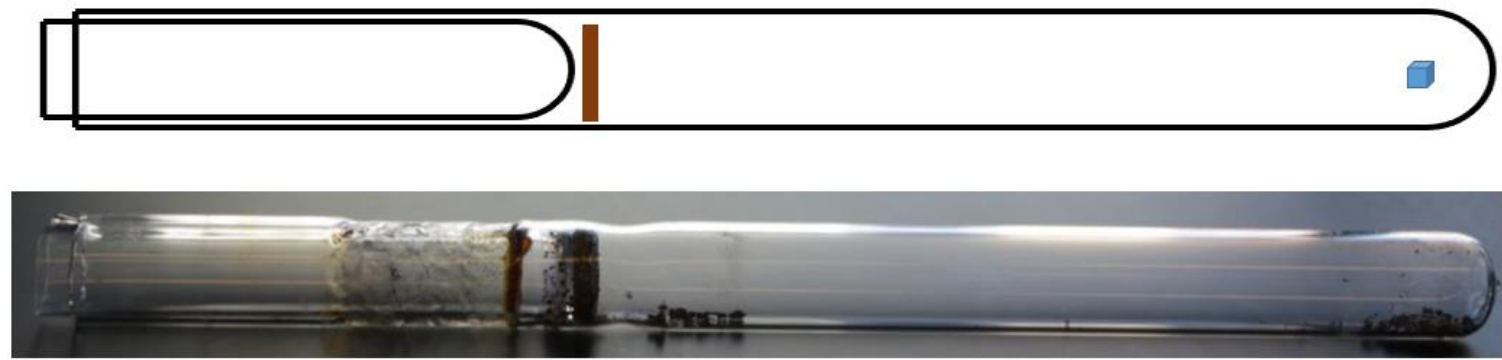

Figure 3.2. Above, a schematic of the homoepitaxial CVT growth. The hot zone is at 650 ${ }^{\circ} \mathrm{C}$ for 12 days. The source is transported to the seed crystal by the addition of $\mathrm{FeBr}_{2}$. Below is a photograph of the ampoule after growth.

Figure 3.2 shows a cartoon and a photograph of the homoepitaxial growth process.

Originally $1 \mathrm{~g}$ of natural pyrite was used as the source with iron bromide $\mathrm{FeBr}_{2}$ as the halide transport agent. The oven set to $650{ }^{\circ} \mathrm{C}$ for 21 days. This resulted in the growth of many crystals at the cool end onto the natural slide. The housing is made of quartz glass.

\subsubsection{HTSG onto CVT}

The HTSG growth onto natural pyrite is repeated here. However, in this instance instead of using the natural slide of pyrite as the growth substrate, CVT pyrite will be used as the growth substrate. Detailed information on the HTSG can be found in section 2.2.6. 


\subsection{Results \& Discussion}

\subsubsection{Natural pyrite as a substrate for HTSG}

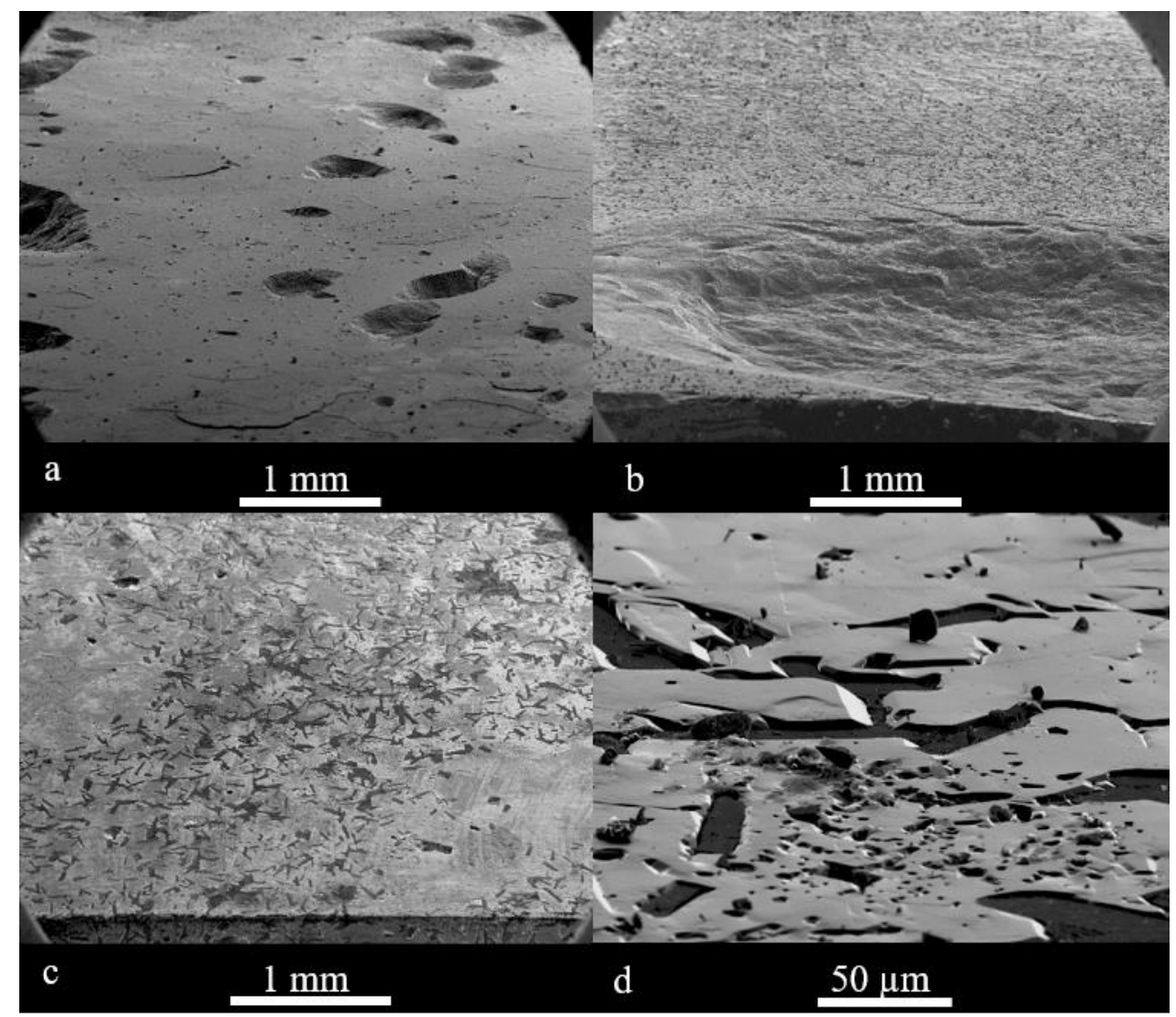

Figure 3.3: SEM of defects in natural pyrite post HTSG.

There is also a visual difference in some of the substrates after growth. Figure 3.3

shows SEM different images of natural pyrite after it has been through the HTSG process.

In Figure 3.3.a,b the surface of the substrate after growth shows very large craters. There are also cracks in the material. Neither of these were present before growth. If these have fallen off during the homogenization of the melt they could compete with the dissolution of the precursor. If they formed during the crystallization they would be a compete site for growth. Either situation makes a repeatable crystal impossible. 
Figure 3.3.c, d show natural defects that are located within the pyrite. These small lines all over the surface. At higher magnification these appear to be underneath the surface layer. EBS of these show that they are comprised of mostly oxygen with large amounts of aluminum and silicon and small amounts of magnesium and sodium. The defects on the surface appear to be underneath the surface layer. This would support the idea that the originally flat surface has had an over layer grown on the pyrite but not the defects. These defects do not allow a repeatable substrate to be used directly from natural pyrite. It is possible that they affect the $V_{\mathrm{ph}}$ measurement. A new source should be used for characterization.

\subsubsection{CVT crystals}

The resulting crystals were ground up and analyzed with XRD.

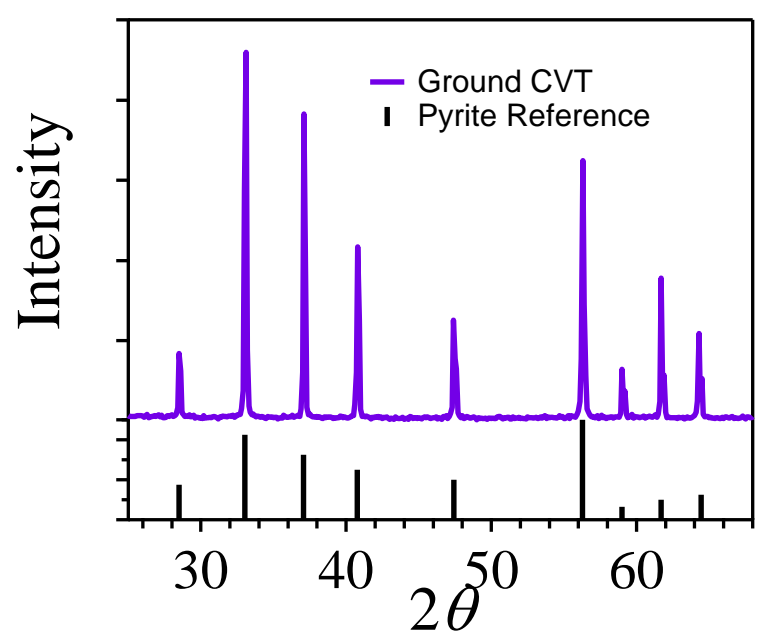

Figure 3.4: XRD pattern of ground up CVT pyrite (purple) with a pyrite reference included (black). 
Figure 3.4 shows the XRD pattern for crushed CVT crystals along with reference peaks for pyrite. All the peaks in the pattern are in the same position as the reference for pyrite. There it can be concluded that the sample is pyrite.

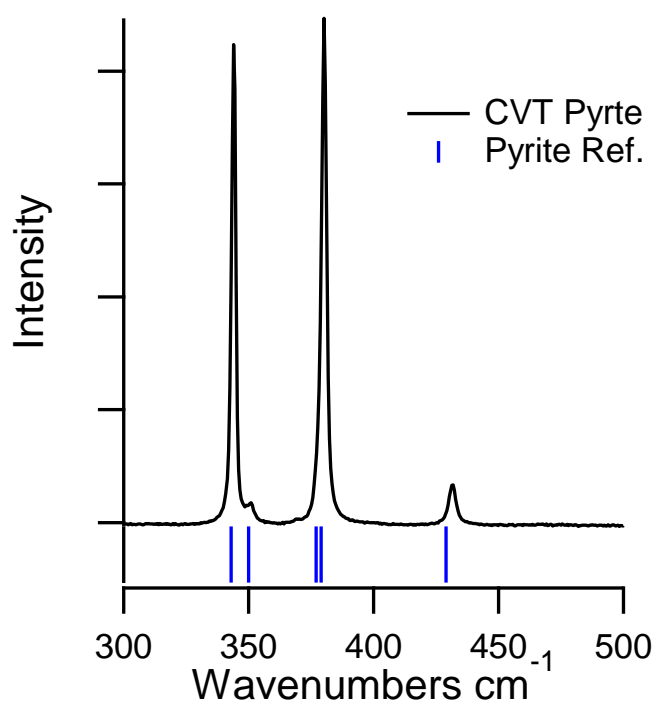

Figure 3.5. Raman spectroscopy of CVT pyrite. A pyrite reference (blue) is included.

Raman spectroscopy was used to analyze the surface of the CVT pyrite before it was crushed to determine if any marcasite was present. The resulting spectrum can be seen in Figure 3.5. The only peaks that appear here correlate to pyrite peaks and are of similar intensity to those shown in the literature.

It can be concluded that the CVT growth with utilizing only one temperature zone is successful in making pyrite crystals. 


\subsubsection{CVT over-layer onto natural pyrite}

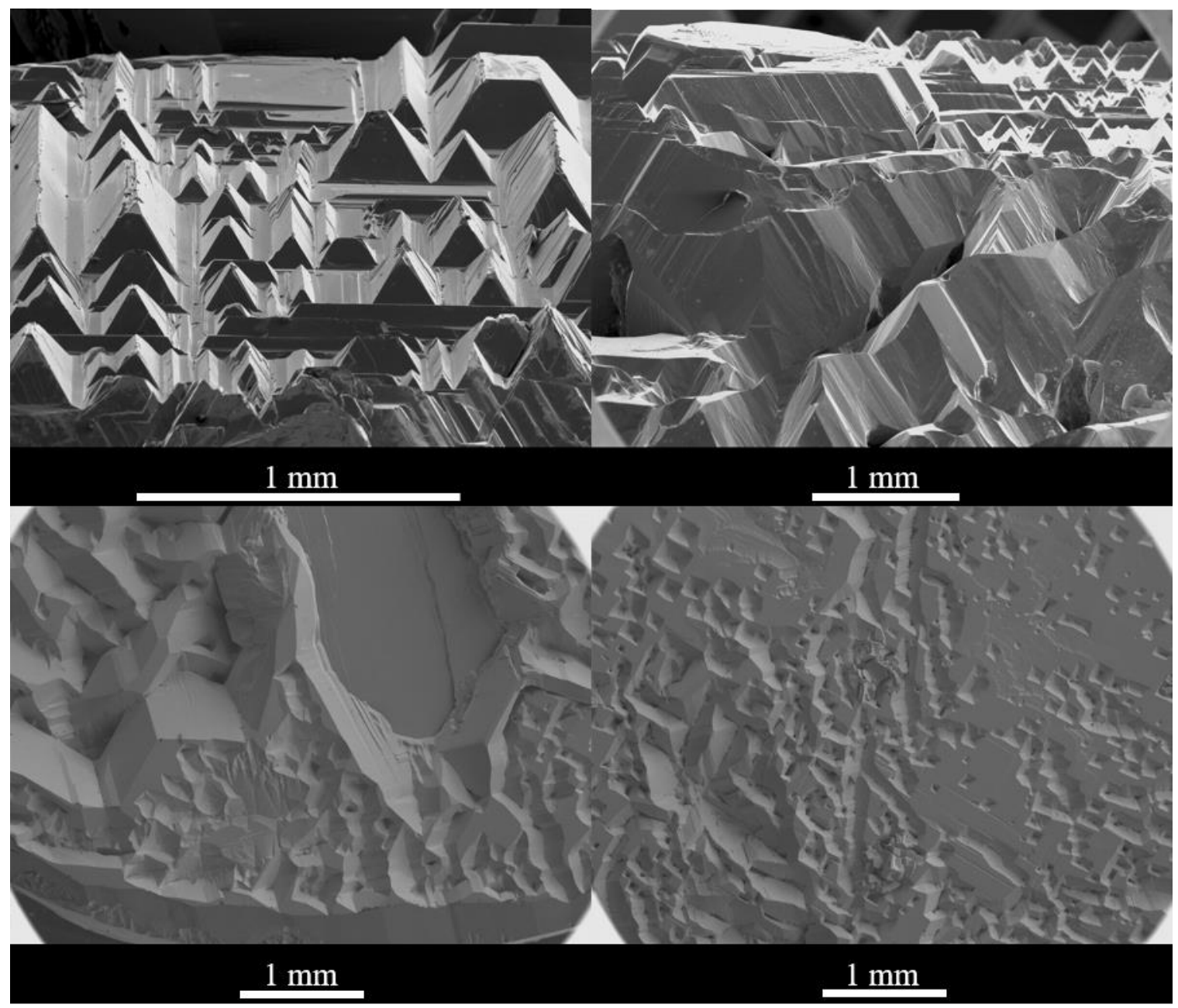

Figure 3.6: CVT pyrite grown onto polished natural pyrite. top is from one growth, bottom is from another.

Figure 3.6 shows SEM images of the result of two different homoepitaxial pyrite growths. One is shown in the top two images and other for the bottom two images. All the CVT growths have resulted in the alteration of the surface of the substrate. The mass of the substrate had also increased after the growth indicating the deposition of material.

Synthetic pyrite has grown onto the surface of the synthetic pyrite. Where this is not always in the same orientation enough can be grown or the process repeated so that the 
substrate can be removed, and the surface can be polished resulting in a flat surface to be used in the HTSG.

\subsubsection{HTSG onto CVT}

The surface of the HTSG onto CVT pyrite after removal of the melt was analyzed with SEM to look for similar morphological features as seen in the HTSG onto natural pyrite.

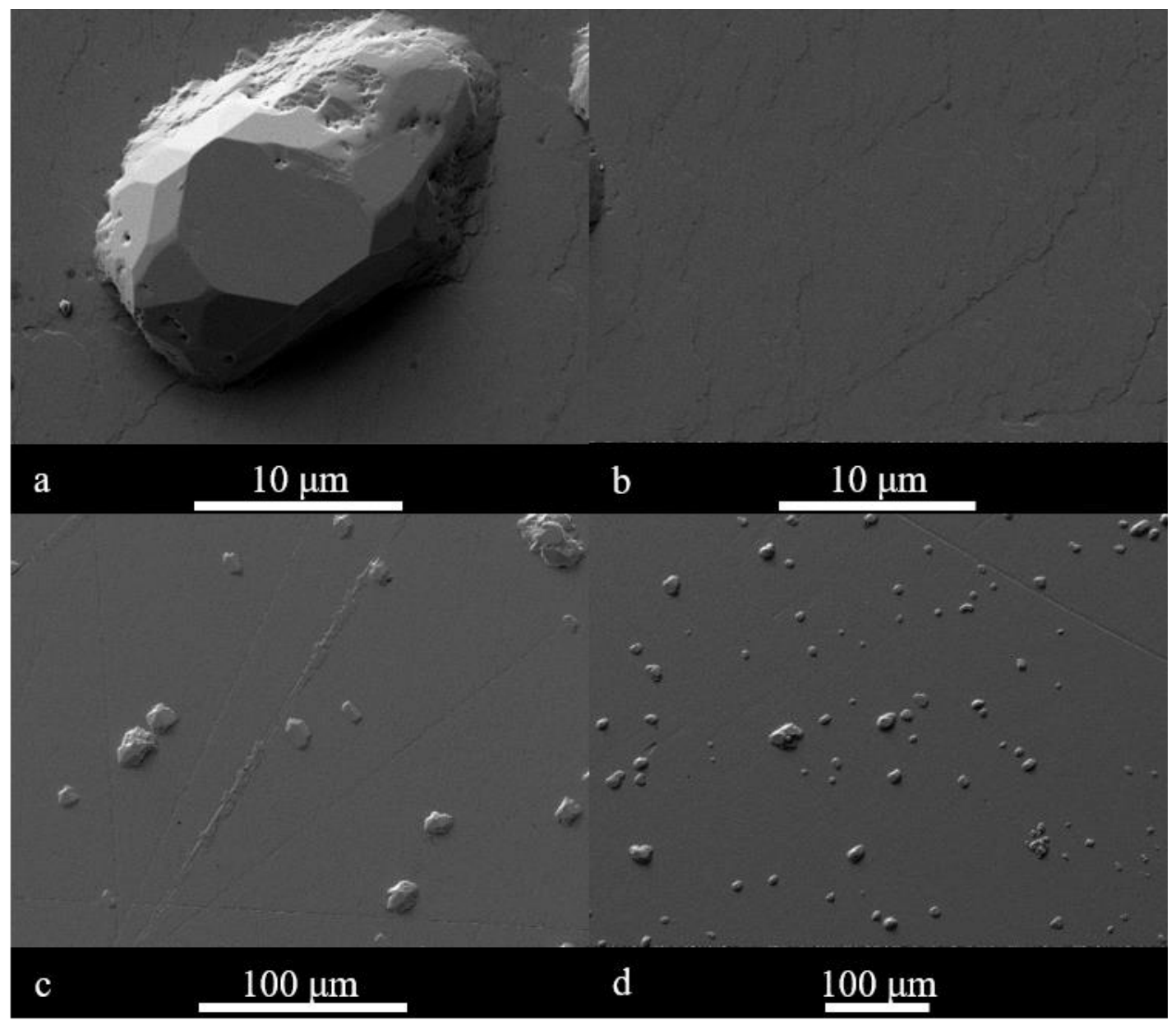

Figure 3.7: HTSG onto a CVT crystal. a-d) are all the surface of the substrate.

Figure 3.7 shows four SEM images of the surface of the substrate of the HTSG onto the CVT pyrite. The mass of the slide has increased because of the growth, material has 
been deposited. There are small crystals that have grown from the surface shown in Figure 3.7.a, c, and d. The surface is no longer flat and has adopted a new surface morphology because of the growth as shown in Figure 3.7.b. There appears to be regions where there is growth over the lines that were once on the surface in effect healing these marks. There was no evidence on the surface of the slide of large pits growing or new cracks forming like the ones seen in the HTSG onto the natural pyrite. This is an indication that this new substrate can be used in the growth without the drawbacks of substrate destruction. This provides a repeatable substrate which could be used to optimize the growth.

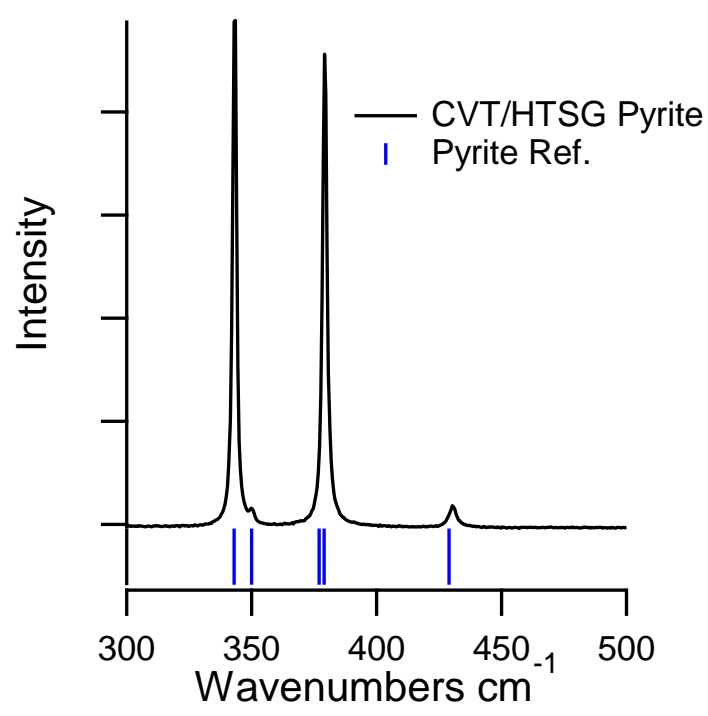

Figure 3.8. Raman of HTSG/CVT. A pyrite reference (blue) is included.

Raman spectroscopy was used to determine if the surface of the growth was still pyrite. This spectrum shown in Figure 3.8 represents the flat surface as well as the top of the crystals on top of the surface. Only peaks representative of pyrite are observed in the spectrum. This is evidence that the material is pyrite and not any of the other iron sulfur species. Now that the over-layer has been confirmed the photovoltage will be measured. 


\subsubsection{Electrochemical characterization of HTSG/CVT pyrite}

After the successful CVT growth resulting in production of a single crystal pyrite sample, the CVT pyrite was tested electrochemically to asses any changes in the PV performance in comparison to natural samples. The photovoltage was measured in the same way as described in section 2.3.6. $V_{\mathrm{ph}}$ data is summarized in Table 3.1. Photovoltage values for each sample, before and after sulfuric acid etch (activation) is required for each pyrite/redox junction and for two illumination intensities. Illumination intensity of 1 Sun and a high illumination intensity ( 4 Sun) was used.

Table 3.1. Photovoltage measurements of HTSG/natural pyrite and HTSG/CVT pyrite samples.

$V_{\mathrm{ph}}$ for pyrite/redox couple junction $(\mathrm{mV})$

\begin{tabular}{|c|c|c|c|c|c|c|c|}
\hline \multirow[b]{2}{*}{ Sample } & \multirow[b]{2}{*}{ Preparation } & \multicolumn{2}{|c|}{$\mathrm{Cp}_{2} \mathrm{Fe} / \mathrm{Cp}_{2} \mathrm{Fe}^{+}$} & \multicolumn{2}{|c|}{$\mathrm{Cp}_{2} \mathrm{Co} / \mathrm{Cp}_{2} \mathrm{Co}^{+}$} & \multicolumn{2}{|c|}{$\mathrm{I}^{-} / \mathrm{I}_{3}^{-}$} \\
\hline & & 1 sun & h. int. & 1 sun & h. int. & 1 sun & h. int. \\
\hline \multirow{2}{*}{$\begin{array}{c}\text { HTSG } \\
\text { on } \\
\text { NAT }\end{array}$} & as is & -7 & - & 0 & - & -9 & - \\
\hline & etched & -7 & - & -1 & - & -6 & - \\
\hline \multirow{2}{*}{$\begin{array}{c}\text { HTSG } \\
\text { on } \\
\text { CVT }\end{array}$} & as is & -4 & -24 & - & - & -5 & -30 \\
\hline & etched & -56 & -117 & -40 & -106 & -50 & -110 \\
\hline
\end{tabular}

The HTSG/CVT pyrite samples consistently show higher photovoltage values when compared to the HTSG/natural pyrite samples especially after the sulfuric acid etching. Overall, synthetically grown CVT crystalline pyrite was shown to have higher PV performance in terms of $V_{\mathrm{ph}}$. To further understand the specifics of these junctions, cyclic voltammetry was used. Current voltage behavior of the HTSG/CVT pyrite is shown in Figure 3.9. 

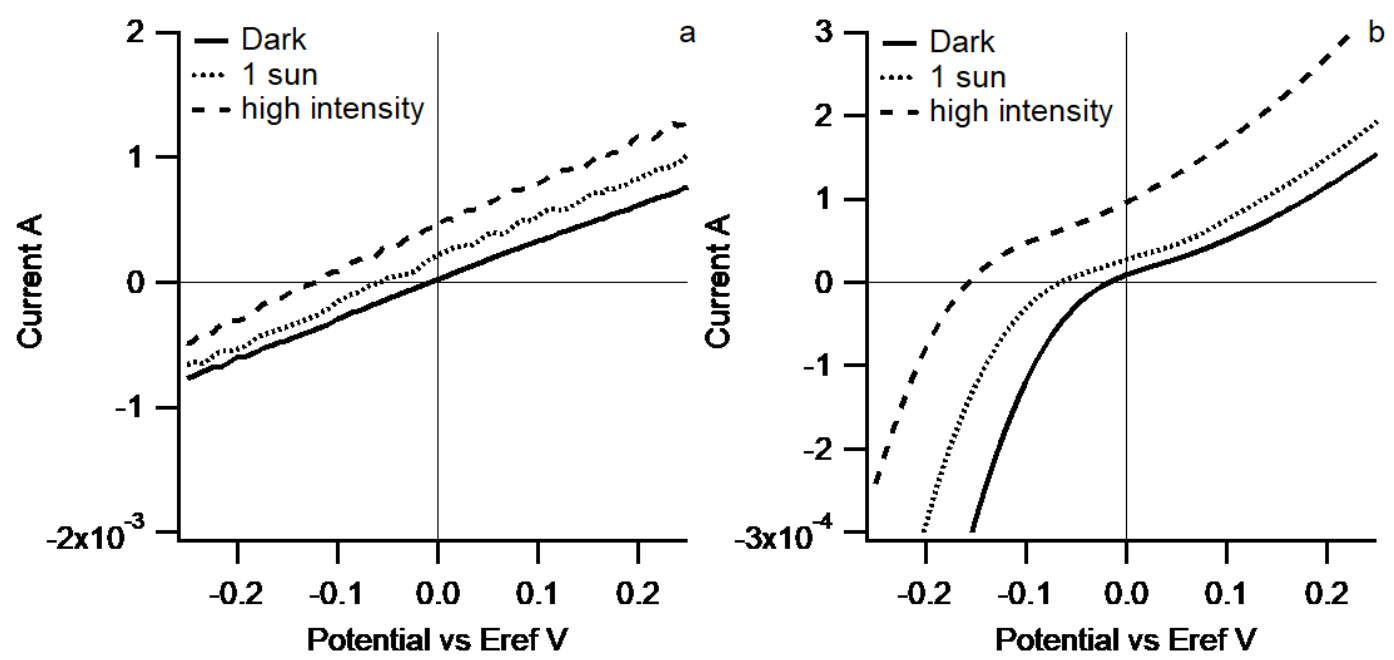

Figure 3.9: $I-V$ curve for etched HTSG/CVT pyrite contacted with a) $\mathrm{Cp}_{2} \mathrm{Fe} / \mathrm{Cp}_{2} \mathrm{Fe}^{+}$, and b) $\mathrm{I}^{-} / \mathrm{I}_{3}^{-}$redox solutions.

Figure 3.9 shows I- $V$ curves for the HTSG/CVT pyrite after the cathodic etching in sulfuric acid. The data was collected in the ferrocene/ferrocenium $\left(\mathrm{Cp}_{2} \mathrm{Fe} / \mathrm{Cp}_{2} \mathrm{Fe}^{+}\right)$(left) and the iodide/triiodide $\left(\mathrm{I}^{-} / \mathrm{I}_{3}^{-}\right)$redox couple (right). Higher FF values of 31.4 were measured for HTSG/CVT pyrite samples contacted with the with $\mathrm{I}^{-} / \mathrm{I}_{3}{ }^{-}$redox couple, while in the case of using the $\mathrm{Cp}_{2} \mathrm{Fe} / \mathrm{Cp}_{2} \mathrm{Fe}^{+}$solution the $\mathrm{FF}$ values were 26.2. This is consistent with higher shunt resistance values measured for pyrite/ $\mathrm{I}^{-} / \mathrm{I}_{3}{ }^{-}$junctions and suggests that the iodide/triiodide solution is potential a better contacting media for investigating the PV properties of pyrite. 


\subsubsection{Effects of sulfuric acid etching on the HTSG/CVT pyrite surface}

To understand specifics of the cathodic etching of pyrite in $\mathrm{H}_{2} \mathrm{SO}_{4}$ the surface of pyrite's morphology was characterized using SEM.

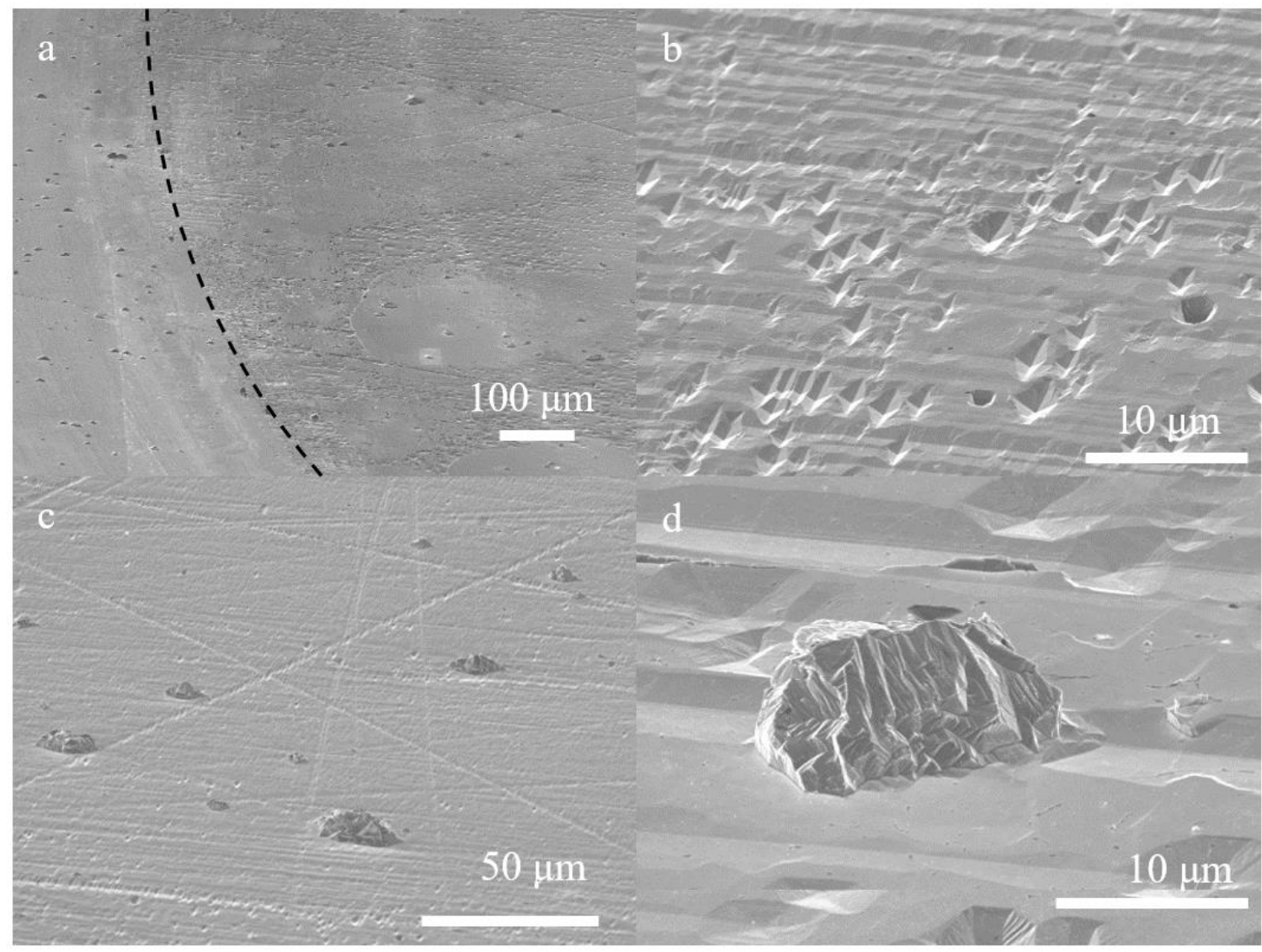

Figure 3.10: Surface HTSG CVT pyrite after electrochemical etching.

Figure 3.10 shows the surface of the HTSG onto CVT pyrite after it had been etched with a cathodic current in sulfuric acid. Figure 3.10.a shows the surface of pyrite. The line drawn on the image shows the approximate boundary where to the left was area covered by the O-ring and the area to the right was exposed to the etching solution. To the right there are different morphologies, smooth flat regions, regions with pits, scattered islands protruding from surface. It appears that the etching was not homogenous on the surface. 
Figure 3.10.b is a higher magnification of pitted region. It appears that the pits are deeper than $200 \mathrm{~nm}$. This is deep enough to remove the entire layer as estimated SIMS depth layer profile of the isotope doped growth described earlier. Figure 3.10.c shows pitted region including remains of mounds. It can be seen here that the lines from the polishing are still distinguishable, appearing deeper than before etching. Figure 3.10.d shows one of the islands from the surface after etching. Material has clearly been removed but some from the growth remains.

Material has been removed from the surface. Some of that had been the HTSG and some likely the CVT substrate underneath. It is likely that the photovoltages measured are characteristic of a mix between these two and not only the HTSG over-layer. To determine this the HTSG would have to be optimized to produce thick enough so that they are not removed from the etching or layers even thicker that can be grown and then their backside removed as was done for the CVT onto natural pyrite growth.

\subsection{Conclusions}

A single temperature zone furnace was used to make CVT pyrite crystals. The same CVT setup was able to be used to make a thick over-layer onto natural pyrite. This process was able to be repeated to make thicker layers that could be modified to flat surfaces to be used in the HTSG. The HTSG layer grown on the CVT crystal was able to produce higher photovoltages than HTSG onto natural pyrite. Whether this is from the HTSG growth layer alone or from a combination of the growth layer and the CVT remains unknown and needs to be investigated further. The bulk of the material has been changed, the next area of focus would be the surface. 


\section{The effect of surface modification on photovoltage}

\subsection{Introduction}

To study the effect of surface modification first the different methods of modification are to be chosen and then the techniques used to characterize them are discussed. The photovoltage measurements will be performed for the described below surface treatments of pyrite to establish their correlations and to understand the path to further advances in PV performances of pyrite.

\subsection{Experimental}

\subsubsection{Materials}

All chemicals were used as received. Single cube pyrite samples were natural and purchased from The Fossil Cartel. Tellurium (Sigma 99.999\%) was received from Sigma Aldrich. Sulfur (Alfa Aesar 99.999\%), and iron (99.99\%) were received from VWR. Iron bromide $\left(\mathrm{FeBr}_{2} 99.995 \%\right)$ was received from Fisher Scientific. Quartz tubing was received from GM Associates. Ethylenediamine (>98\%), ethanethiol (>98.0\%) were received from VWR. Concentrated sulfuric acid (ACS grade), ferrocene (98\%), and ferrocenium tetrafluoroborate (technical grade), potassium iodide $(99 \%)$, iodine $(>99.8 \%)$ and cobaltocene $\left(\mathrm{Cp}_{2} \mathrm{Co}\right.$, \%) were purchased from Sigma-Aldrich. Cobaltocenium hexafluorophosphate $\left(\mathrm{Cp}_{2} \mathrm{CoPF}_{6},>95.0 \%(\mathrm{~T})\right)$ was purchased from TCI America. Acetonitrile (>99.8\%, anhydrous) was purchased from EMD. Lithium perchlorate (99.99\%, anhydrous) was purchased from VWR. Ferrocene was purified by sublimation. All chemicals except sulfuric acid were stored in a nitrogen-filled glovebox (LABstar MBRAUN, less than 0.5 ppm $\mathrm{O}_{2}$ and $\mathrm{H}_{2} \mathrm{O}$ ). 


\subsubsection{Instrumentation}

The photoelectron spectroscopy system used for this research was a PHI 5000 Versaprobe II utilizing an Al K $\alpha$ X-ray beam (1486.6 eV) was used for all XPS. A helium discharge lamp included in the XPS setup was used for UPS data collection. All samples referenced to $\mathrm{Au}$ foil which was sputtered and used to calibrate the binding energy of the UPS spectra. The onset and electron cutoff were determined by finding the intersection of the slope and the line before or after it. All photoelectron spectra were processed with PHI Multipak version 9.5.0.8.

\subsubsection{Sample Histories}

Four different sample histories of CVT crystal surfaces were studied to correlate their surfaces with their photovoltages. (1) a CVT crystal that had been polished with a series of diamond lapping films in decreasing roughness to produce a flat surface. (2) a CVT crystal that had undergone the HTSG to produce an over-layer that has previously been described. (3) a CVT crystal of pyrite that had undergone the cathodic etch previously described where it was immersed in $0.5 \mathrm{M} \mathrm{H}_{2} \mathrm{SO}_{4}$ and held at a potential of $-1.1 \mathrm{~V}$ vs ref. until a total charge of $1.8 \mathrm{C}$ had passed. (4) a crystal of CVT pyrite that had been heated to $650{ }^{\circ} \mathrm{C}$ where it stayed for 12 hours in an evacuated ampoule that contained elemental sulfur. This sulfur annealing step has been studied previously and is described below. ${ }^{115}$

\subsubsection{Sulfur anneal}

Slides of natural pyrite have been prepared using polishing methods and were then annealed in a sulfur atmosphere which led to an improvement of optical properties as well as surface crystallinity as determined by EBSD. This led to a repeatable pyrite surface 
where there had not been a previous method to produce repeatable surface properties. In this instance the current author contributed to the research by supplying the means by which to anneal the pyrite as well as assistance in preparing the manuscript. Table 4.1 and Figure 4.1 are from the paper showing dielectric functions, and EBSD patterns. The details of the experiments were described in a published article. ${ }^{115}$ The dielectric functions became more repeatable after the annealing. EBSD is a technique used with SEM that detects backscattered electrons from the sample on a phosphor screen to create Kikuchi diffraction patterns that index as different crystal systems. This technique gives us surface crystallinity. The indexing values of the Kikuchi patterns by EBSD for natural samples of pyrite have improved after the sulfur annealing. The Kikuchi pattern images can be seen below. Because of the improvement of the near surface and as an attempt to fill near surface sulfur vacancies this technique was selected to create one of the CVT substrate surfaces.

Table 4.1. The dielectric functions of pyrite slides before and after annealing in a sulfur atmosphere (each data was an average of five spots on the same slide).

\begin{tabular}{ccccc}
\hline & \multicolumn{2}{c}{ Pre annealing } & \multicolumn{2}{c}{ Post annealing } \\
\cline { 2 - 5 } & $n_{0}$ & $k_{0}$ & $n_{0}$ & $k_{0}$ \\
\hline Slide 1 & $3.98 \pm 0.18$ & $-3.10 \pm 0.02$ & $4.05 \pm 0.16$ & $-2.86 \pm 0.04$ \\
Slide 2 & $3.25 \pm 0.03$ & $-3.03 \pm 0.01$ & $4.43 \pm 0.12$ & $-3.02 \pm 0.03$ \\
Slide 3 & $1.79 \pm 0.04$ & $-3.29 \pm 0.02$ & $4.54 \pm 0.02$ & $-2.96 \pm 0.02$ \\
\hline
\end{tabular}


(a)

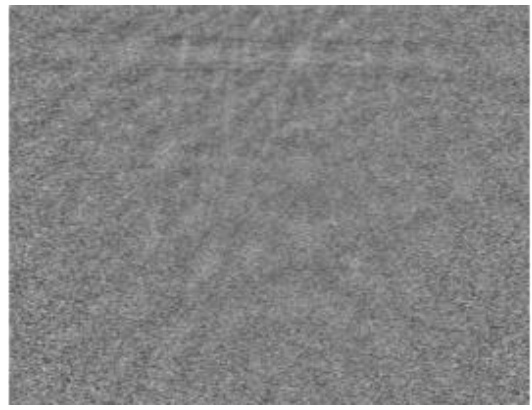

(c)

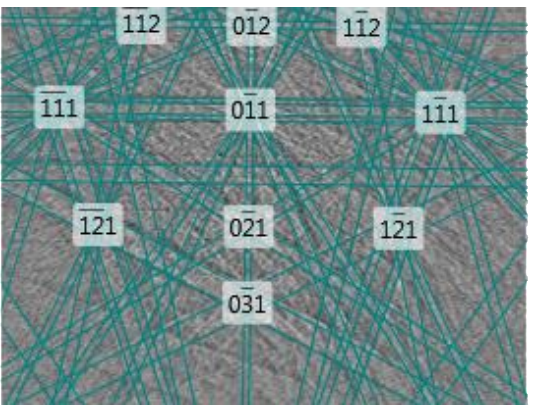

(b)

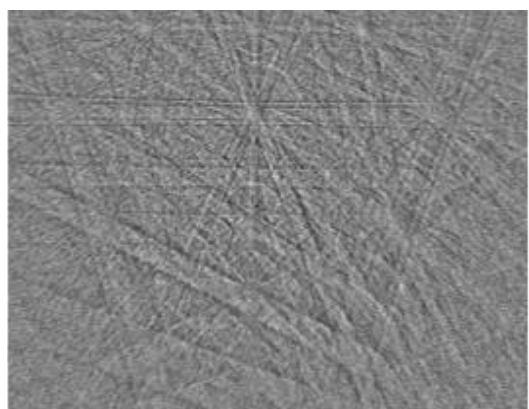

(d)

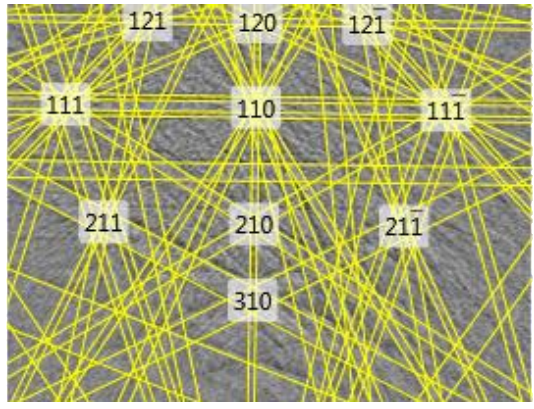

Figure 4.1. EBSP (Kikuchi patterns) of a pyrite slide (a) pre- and (b) post-sulfur anneal.

(c) and (d) are auto index results of two different spots on the annealed slide.

\subsection{Results \& Discussion}

\subsubsection{X-Ray photoelectron spectroscopy study of different pyrite surfaces}

X-ray photoelectron spectroscopy uses a beam of x-rays to remove electrons from the core shell of atoms. These photoelectrons have their kinetic energy measured with an analyzer. The difference of the kinetic energy of the photoelectron and the energy of the incident beam is equal to the binding energy of the electron. The binding energy of the core electrons of atoms shift according to different chemical environments that the atom is in. Typically, the more oxidized an atom is the higher the binding energy is of the core electrons. This is because the total attraction of the nucleus to the electrons is now divided up among fewer electrons. If an atom is more reduced the binding energy will typically be smaller because there are now more electrons to divide the attraction of the nucleus among. There are some cases where this trend is not followed due to inter atomic effects of 
surrounding atoms. This method allows the user to identify not only that atoms present at the near surface of the material $(5-10 \mathrm{~nm})$ but their chemical state as well. The data obtained from this technique is not as straightforward as it sounds. Each element has its own sensitivity to the generation of photoelectrons. The binding energy of the electronic levels also determines how surface sensitive they are. With a higher binding energy, the kinetic energy of the photoelectron is smaller, and the electron has a decreased likelihood of reaching the surface from deeper within the sample. With a lower binding energy and higher kinetic energy electrons from deeper within the sample can reach the surface without losing energy and can make their way to the detector. 


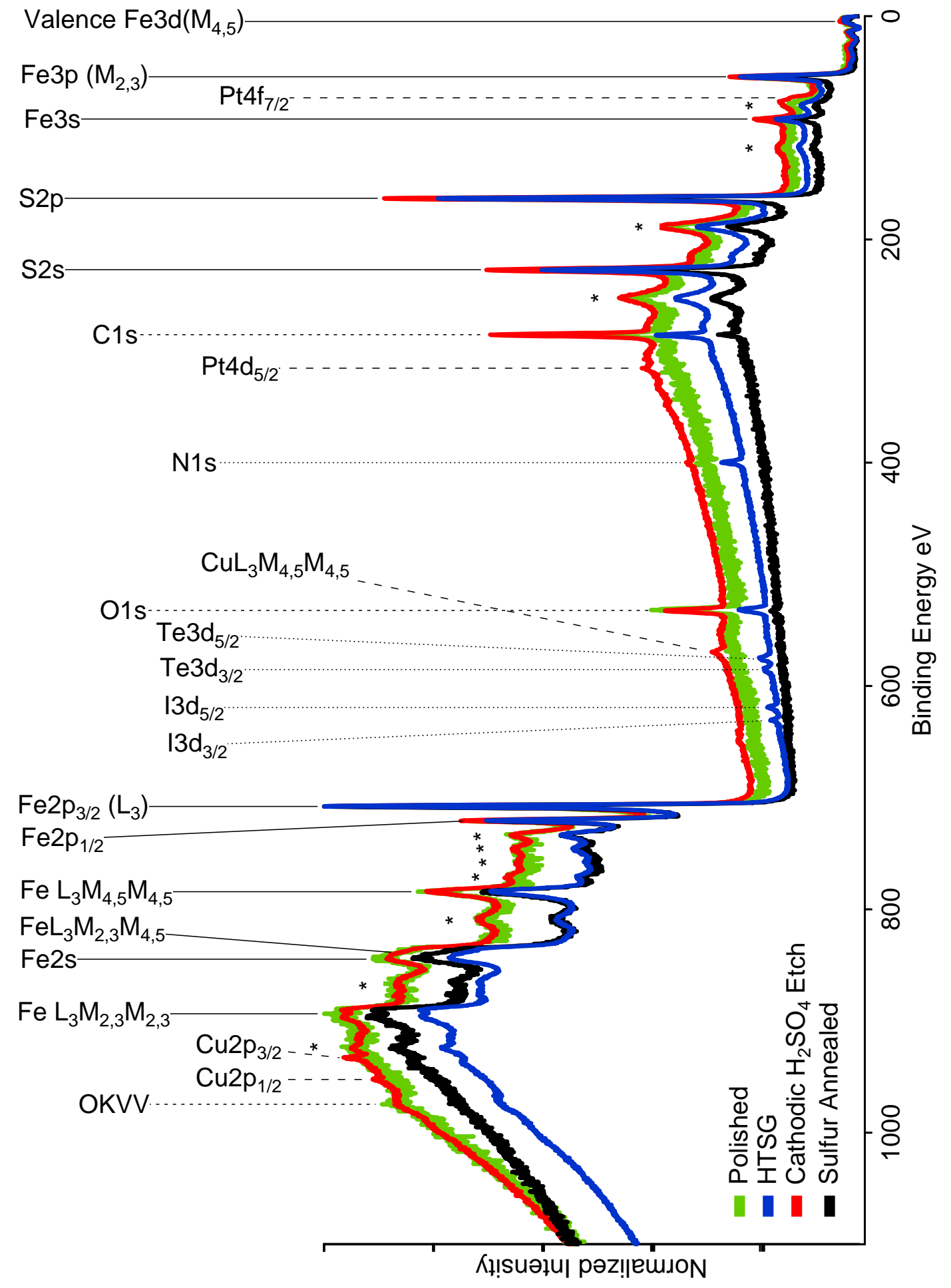

Figure 4.2: XPS survey scan of polished, sulfur annealed, etched and HTSG CVT pyrite. 
XPS survey scan of CVT pyrite surfaces that have been polished, through HTSG, through a cathodic etch in sulfuric acid, and sulfur annealed all show peaks corresponding to iron and sulfur peaks from their electronic configuration. This is observed in Figure 4.2. They also have peaks for iron auger peaks. When a photoelectron is emitted there is a whole left in the orbital. Electrons from smaller binding energies can fill these wholes. When they do so they can donate their energy to electrons from the same or lower binding energies. If of high enough energy these electrons can now exit the material. They make their way to the detector where their kinetic energies are recorded and correlated to a binding energy. There are no actual electrons at these binding energies. The label $\mathrm{L}_{3} \mathrm{M}_{2,3} \mathrm{M}_{4,5}$ represents a photoelectron leaving the $\mathrm{L}_{3}$ level, an electron from $\mathrm{M}_{2,3}$ replacing the electron and an electron from $\mathrm{M}_{4,5}$ leaving the atom. There are also energy loss peaks about $25 \mathrm{eV}$ higher in binding energy for the iron and sulfur peaks. These may result from a cost of energy to leaving the bulk due to oscillations of electrons in the conduction band. They show up in reference XPS spectra for elemental iron and sulfur and are characteristic of the atoms themselves and are not a result of the specific chemistry of these samples. All spectra show peaks for carbon and oxygen which are most likely due to adventitious carbon on the sample surface

The HTSG spectra has addition peaks for nitrogen tellurium and iodine. Nitrogen is most likely from the removal of the solidified melt with ethylenediamine, tellurium from the melt, and iodine from handling in the glovebox after an iodine experiment. The cathode etch in sulfuric acid sample has additional peaks for copper and platinum. These may be a result of the cathode potential placed on the electrode where copper and platinum can be 
reduced out of solution on to the electrode surface. It was possible to add copper sulfate into the etching solution and increase the amount of copper deposited. Doing so did not show an improvement of the photovoltage. The sulfur annealed spectrum shows no other peaks than those that were already mentioned.

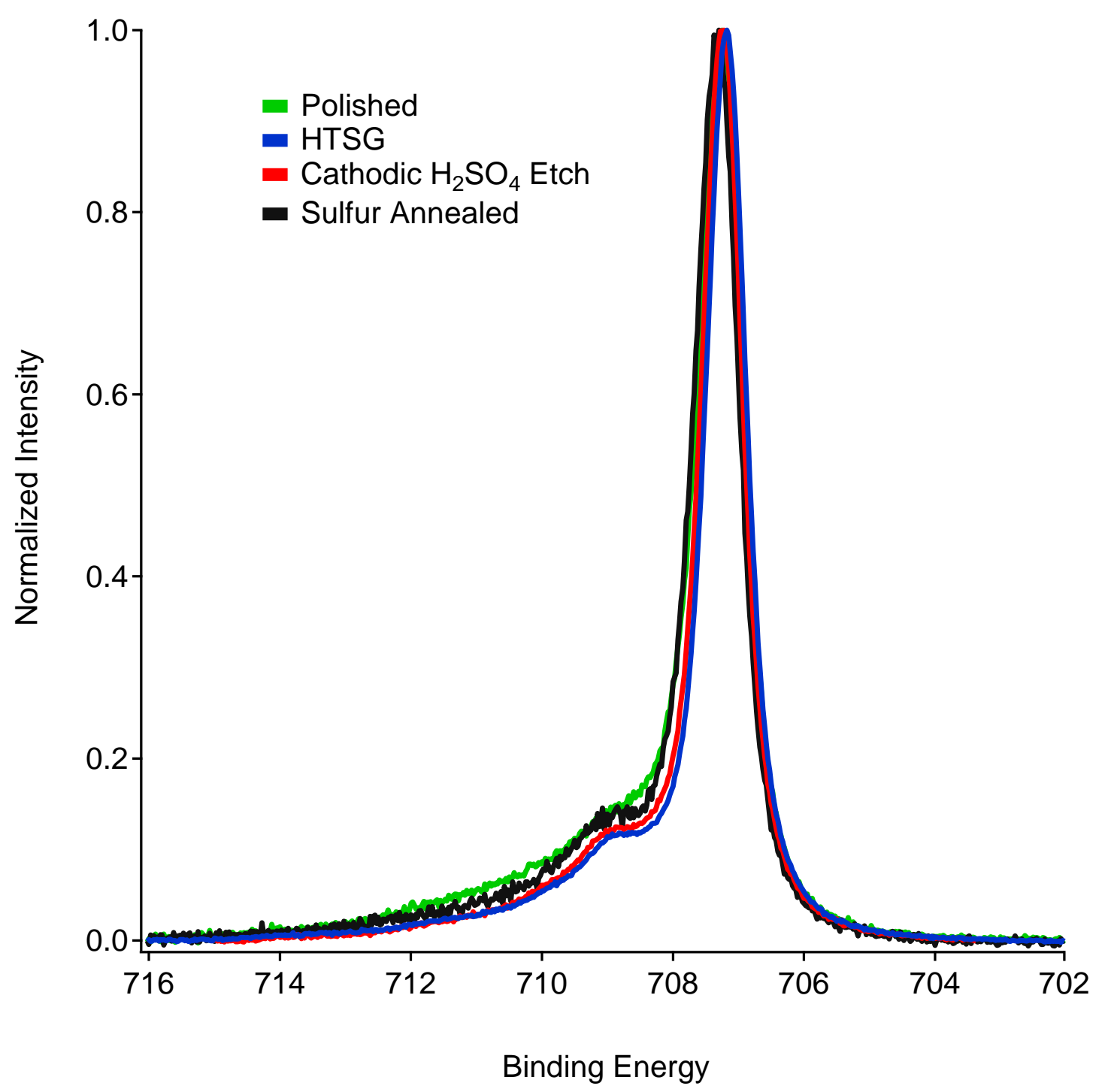

Figure 4.3: Normalized and background subtracted XPS spectra of the Fe2p3/2 range of polished, HTSG, etched in $\mathrm{H}_{2} \mathrm{SO}_{4}$ with a cathodic potential applied and sulfur annealed, CVT pyrite. 
Figure 4.3 shows normalized and background subtracted XPS Fe2p3/2 spectra of polished, sulfur annealed, etched and HTSG CVT pyrite. All four spectra show a large peak near 707.20. This peak represents the iron which is in an octahedral coordination to six sulfur dumbbells in pyrite. All also show a tail extending from the peak into larger binding energies. This should represent ion in a more oxidized environment. It may be possible that this represents an iron that was coordinated to six sulfur dumbbells and then one of those dumbbells had the bond between its two sulfur atoms cleaved. Which could result in the sulfur atom which would now be at a +1 oxidation state oxidizing the $\mathrm{Fe}(\mathrm{II})$ to $\mathrm{Fe}(\mathrm{III})$ itself now with a -2 oxidation state. There is also evidence in the literature that these peaks may be the result of multiplet peak splitting of iron. ${ }^{142-148}$

Electrons in atomic orbitals of atoms with the $l>0$ can couple their spin with their orbit angular moment described by the phenomenon of spin-orbit coupling. This splits the energy level of the orbital into two discrete levels. Multiplet peak splitting is similar but in this case electron spins are coupling to create multiple energy levels. When a hole is generated in an inner shell orbital the remaining electron in that orbital has a magnetic field given by its spin that is either aligned or opposed by the magnetic field of an electron in the valence band. The lone electron in the valence orbital could have been there originally as in a paramagnetic ion or could have been generated in a diamagnetic ion because of the photoelectron generation. The splitting generates peaks of higher binding energy to the main peak. This phenomenon affects orbitals of all $l$ values. The closer in energy to the valence band the orbital is, the larger the difference in energy from the main peak and the 
multiplet peak. This phenomenon has been seen in different iron oxide's $2 p$ and $3 s$ XPS peaks. ${ }^{149}$

It has been proposed that the tail at higher binding energies to the $\mathrm{Fe} 2 \mathrm{p} 3 / 2$ pyrite peak is a result of this phenomenon. ${ }^{142}$ Peaks in that study were fit as a result of mulitplet peak splitting between the Fe2p3/2 orbitals and paramagnetic surface states not from pyrite, but from surface states of $\mathrm{Fe}$ in pyrite that had one $\mathrm{Fe}-\mathrm{S}$ bond cleaved leaving an $\mathrm{Fe}(\mathrm{II})$ atom, and Fe in pyrite with one of the sulfur to sulfur bonds cleaved from its surface facing direction resulting in the oxidation of the iron to $\mathrm{Fe}(\mathrm{III})$ and reduction of the sulfur to -2 . The source of the paramagnetism of the valence band was further explored further by Nesbitt. ${ }^{143}$

There the idea was that originally the iron in an octahedral coordination with the six sulfur dumbbells resulted in a splitting of the $d$ where the $t_{2 g}$ orbitals makeup the valence band edge and the $e_{g}$ orbitals rise to makeup the conduction band edge. With the cleavage of the Fe-S bond the remaining $\mathrm{Fe}$ is no longer in an octahedral coordination. Now the $\mathrm{d}_{\mathrm{z}}{ }^{2}$ orbital fall to within the original bandgap from the conduction band, the $\mathrm{dx}_{\mathrm{x}}^{2}-\mathrm{y}^{2}$ rise, and the $d_{y z}$ orbitals rise from what was once the valence band to a level within the band gap. The $d_{y z}$ orbitals were filled with electrons. However, the distance between the now in gap states being only $0.35 \mathrm{eV}$ is lower than the pairing energy of $1.6 \mathrm{eV}$ of the two electrons in the $\mathrm{d}_{\mathrm{yz}}$ orbital so that they are not both singly filled with electrons in a high spin configuration. These provide two different electrons in the valence band to couple with the electrons in $2 \mathrm{p}$ orbital to couple with. That is from the first surface state. 
The second surface state is from the cleaving of the S-S bond resulting in $\mathrm{Fe}(\mathrm{III})$ bonded to a single sulfur and five sulfur dumbbells. The situation is like the one described above with the difference in the bonding between the sulfur dumbbell and the single sulfur enough to result in a distortion of an octahedral coordination to closer to a square pyramidal. However, in this case the iron is oxidized so that there is one less electron and now a total of three lone electrons. This provides three orbitals that can couple with the Fe2p states. This led those researches to fit the higher binding energy tail with seven peaks from multiplet splitting.

In another study it was shown that when pyrite had been exposed to air to study its oxidation these multiplet peaks could be fit as well. In this case it peaks were fit for the multiplet peaks as well for peaks representing the bond between iron and oxygen at higher binding energies. ${ }^{144}$

Synchrotron XPS was then used to investigate the multiplet splitting. ${ }^{145}$ Here the energy of the incident X-ray beam was $800 \mathrm{eV}$. This excitation energy is more surface sensitive because the kinetic energy of the generated photoelectrons is lower, so they have a lower probability of exiting the surface the deeper in the material they originate. In this case the ratio of the main pyrite peak to the tail lowered so that the tail main peak appeared larger, but the tail stayed the same size. This is evidence that the source of the tail is from the surface and not inherent to pure pyrite. Therefore, even if it is not coming from multiplet splitting caused by surface defects, it is still a signal originating from the surface. Attempts were made to use similar fitting parameters as the previously mentioned studies to fit the tail of the iron XPS peak but could not be done in a consistent and satisfactory manner 
across all samples. It could be that the tail is the result of a is mix of surface states generating peaks as well as multiplet splitting caused by the surface states and that none of ours matched with those in the literature because of different surface preparations.

The four spectra show very similar shape. Each with the large pyrite band and the tail. From this observation one would expect that the photovoltage measurements of each would be similar as their surfaces are of a similar composition. If any difference increasing photovoltage should follow the reverse order of the height of the tail, more surface defects leading to a lower photovoltage. 


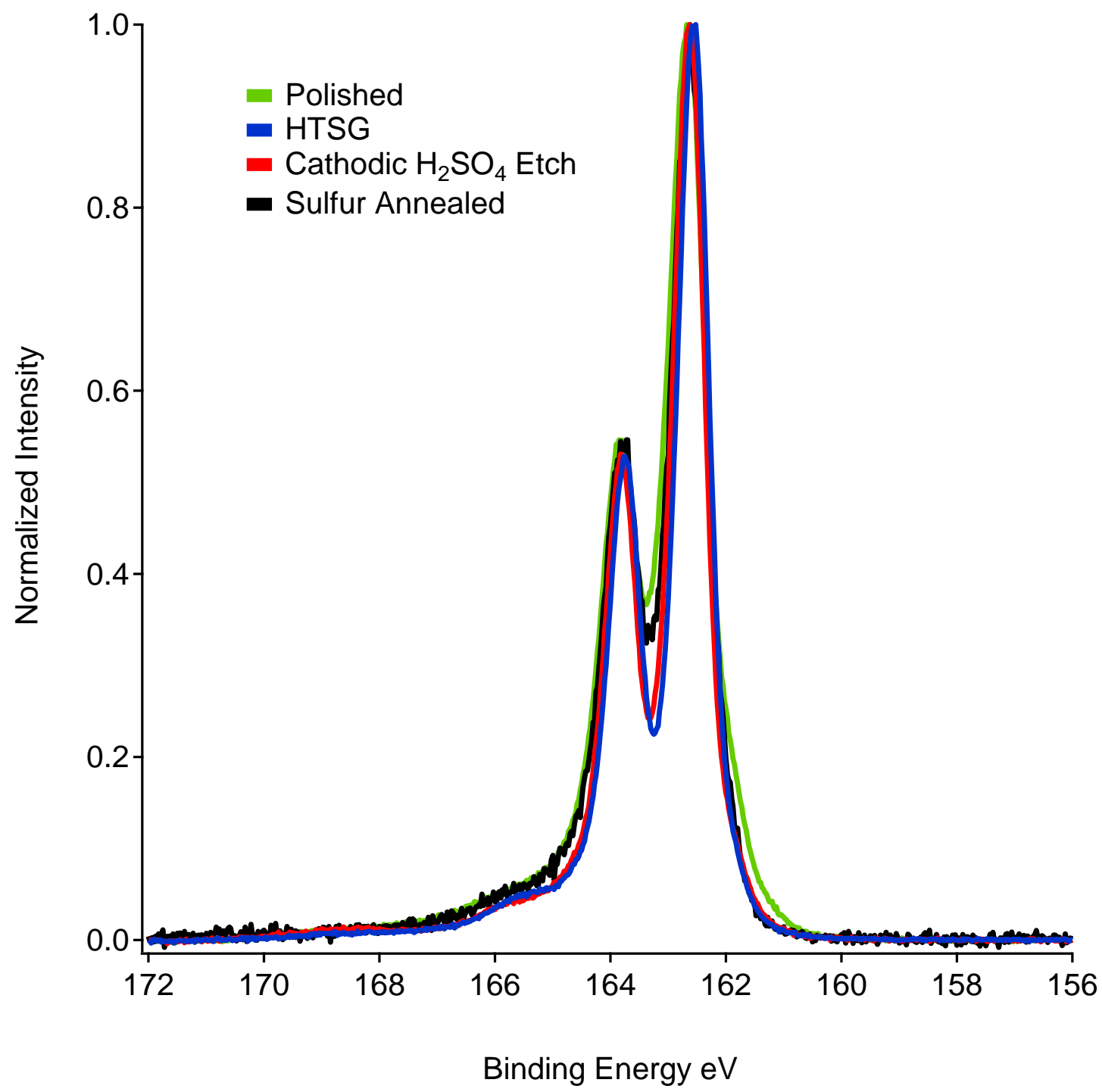

Figure 4.4. Normalized and background subtracted XPS spectra of the S2p range of polished, HTSG, etched in $\mathrm{H}_{2} \mathrm{SO}_{4}$ with a cathodic potential applied and sulfur annealed, CVT pyrite.

Figure 4.4 shows normalized and background subtracted XPS S2p spectra of polished, sulfur annealed, etched and HTSG CVT pyrite. All spectra show the largest peak around 162.60 and a smaller one around 163.8. All also show a tail into higher binding energies. Sulfur $2 p$ orbitals exhibit spin orbit coupling resulting in a separation of the $3 / 2$ and $1 / 22 \mathrm{p}$ peaks of around $1.2 \mathrm{eV}$. This is consistent with the two large peaks seen here. 
These are attributed to the sulfur in pyrite. The small peak at around 165.5 is attributed to polysulfide. It is of a larger full width half max than the main peaks which suggests that it is from the surface of the material and not the bulk. In the iron $2 p$ spectra there were peaks attributed to surface defects. Here there are no obvious such peaks. The photoelectrons generated from the $2 p$ orbitals in sulfur are of a much higher kinetic energy than the iron $2 p$ orbitals. This results in the sulfur spectra receiving a greater proportion of its signal from the bulk of the material and not a signal from the uppermost surface. Higher binding energy photoelectrons are more surface sensitive. In the literature synchrotron XPS was done at a lower beam energy. ${ }^{18,112,143-148,150-152,153,135}$ This results in a lower kinetic energy of the generated photoelectrons, thus it is more surface sensitive. In all cases more peaks were seen in the spectra. In all cases two more sets of $2 p$ peaks could be fit. A set comprising of the $2 \mathrm{p} 3 / 2$ and $2 \mathrm{p} 1 / 2$. These are both located at lower binding energies than the largest peak, the same one seen in my spectra. Being at lower binding energies they are in a more reduced environment. Commonly the lowest binding energy $2 \mathrm{p} 3 / 2$ peak around $161.4 \mathrm{eV}$ is attributed to sulfur because of a sulfur-sulfur bond cleavage resulting in the oxidation of iron to $3+$ and the reduction of sulfur to $2+$. In the pyrite structure sulfur is coordinated in a tetrahedral. Three bonds to different iron atoms and one to the other sulfur making up the dumbbell. The intermediate $2 \mathrm{p} 3 / 2$ peak is around $162 \mathrm{eV}$ and represents a sulfur that has had a bond cleaved to one of its bound irons. With one less iron to share electrons with this sulfur is slightly reduced. It is probable that there are sulfur deficient defect on the surface of our synthetic sample, however not detectable with low surface sensitivity to sulfur for our setup. There is also little difference in the shapes of these peaks 
suggesting that there should be no major difference in the photovoltage measurement attributed to the surface.

\subsubsection{Ultraviolet photoelectron spectroscopy of different pyrite surfaces}

Ultraviolet photoelectron spectroscopy is like XPS in that it uses a beam of photons to remove electrons from atoms and then measure their kinetic energy to determine their binding energy. There are two different He emission lines used for this technique, He1 $(21.22 \mathrm{eV})$ and $\mathrm{He} 2(40.81 \mathrm{eV})$. Because the energy of the incident beam is much smaller this removes electrons mostly from the valence orbitals of the sample. This technique allows the user to determine the work function of a material as well as the difference in energy of the Fermi level and the valence band of a semiconductor. The more n-type a semiconductor is the larger the difference and the more p-type a semiconductor is the smaller the difference. 


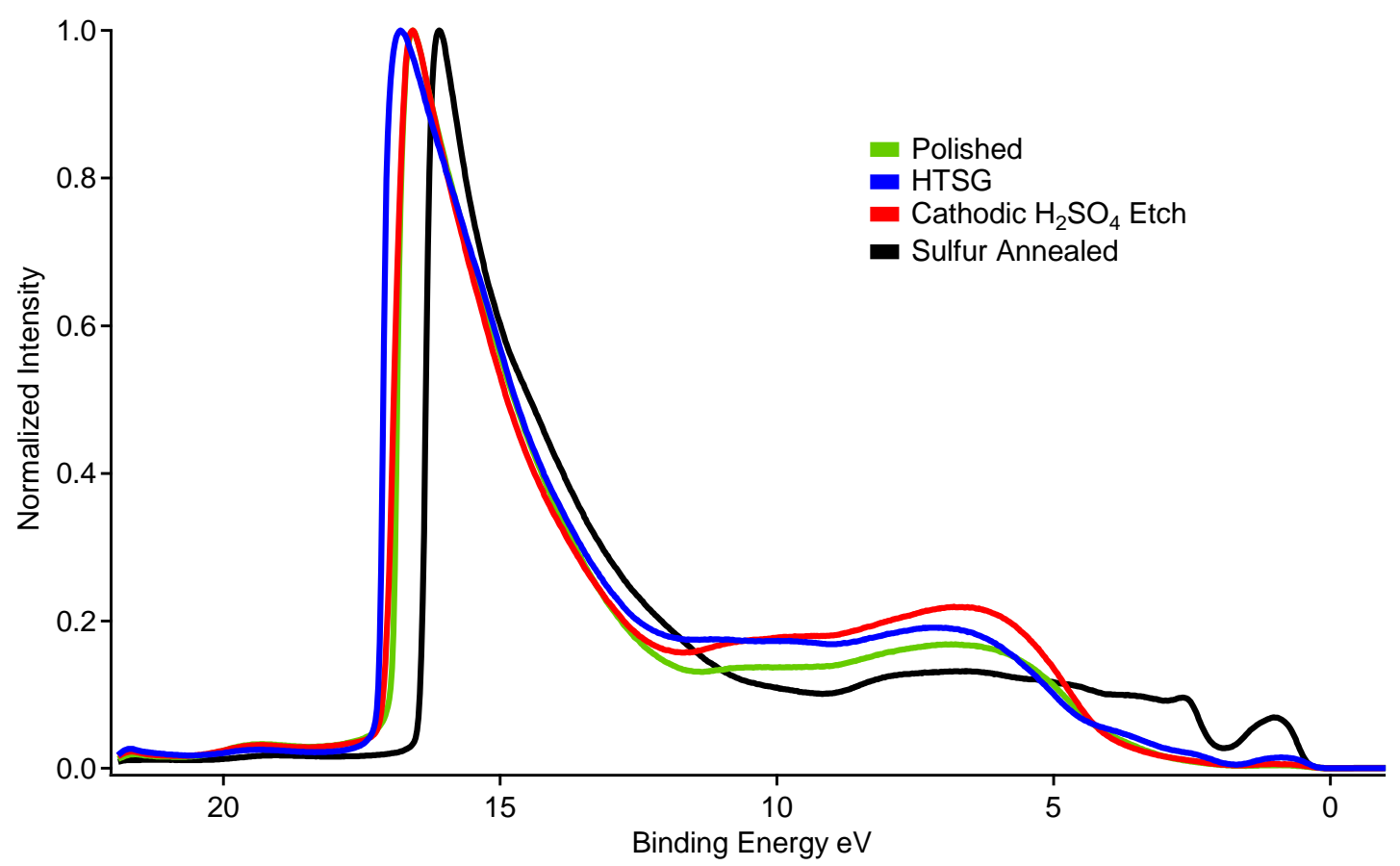

Figure 4.5: Normalized UPS spectra of CVT pyrites with 4 different sample histories, polished, through HTSG, through a cathodic etch in $0.5 \mathrm{M} \mathrm{H}_{2} \mathrm{SO}_{4}$ and sulfur annealed at $650{ }^{\circ} \mathrm{C}$ for 12 hours.

Figure 4.5 shows the UPS HeI spectra for polished, sulfur annealed, etched, and HTSG CVT pyrite. The polished, HTSG, and etched spectra all look similar. They all have very small peaks near the Fermi level and then each slowly increases to a larger peak which centers around $6.5 \mathrm{eV}$ and then remain somewhat flat and then increase sharply and then fall off to very small counts. The larger increase around $12 \mathrm{eV}$ when going to higher binding energies is attributed to inelastically scattered electrons. The reason for their increase is that the detector has been biased $5 \mathrm{~V}$ lower than the sample to increase the probability of detecting photoelectrons with energies around vacuum level. The cutoff for the sulfur annealed sample is at a lower binding energy. This indicates that the sample has a higher work function. The shape of the sulfur annealed spectrum has more definition to it and more closely resembles the UPS spectra from the surface of pyrite that had been 
fractured in the introduction chamber of the UPS. ${ }^{135}$ Another study was done in order to identify the peaks from the UPS spectrum. ${ }^{154}$ Here Different excitation energies were used with XPS and UPS to create spectra for pyrite. Using the different photoionization $\mathrm{x}$ section of the photon energies for elemental subshells (S3s, S3p, Fe3d) they were able to determine the differences in sensitivity at each incident beam energy and compare the spectra across different incident beam energies to determine if the peaks were more dependent on iron or sulfur and then estimate which molecular orbitals each peak represented. They also used calculations from the literature to verify or modify their estimation. ${ }^{155}$ These match well with the spectrum here from the sulfur annealed pyrite. The low binding energy region of the spectra can be used to determine the $n$ - or p-type character of the samples. 


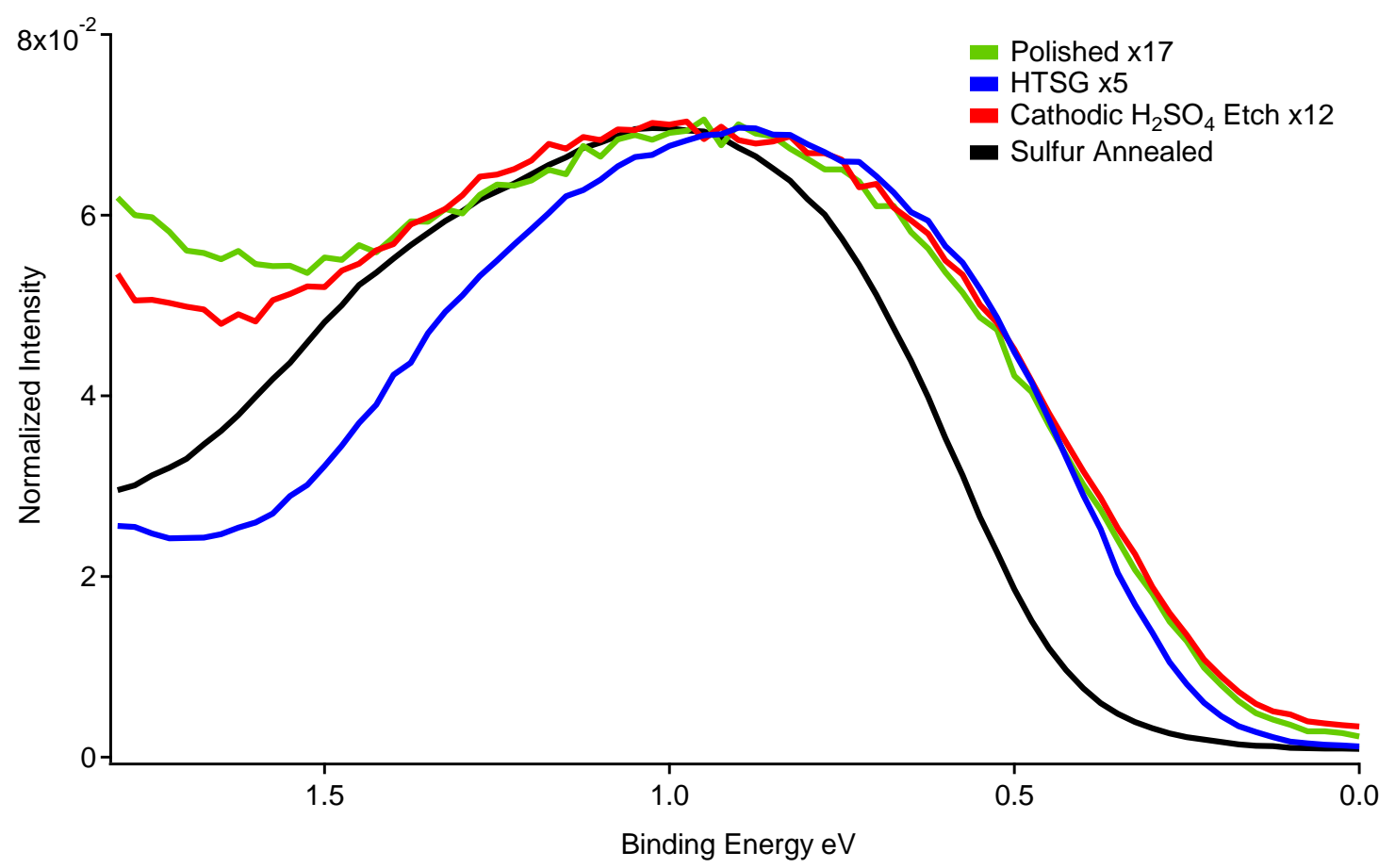

Figure 4.6. UPS spectra of the outer valence band of the CVT pyrite through different histories, polished, HTSG, etched, and sulfur annealed. The spectra have been normalized so that the peak height is roughly the same.

Figure 4.6 shows the UPS spectra of the outer valence band of the four different CVT pyrite sample histories. The height has been normalized to compare the $n$ - and p-type character of each sample. The polished and etched are similar in shape. They are also the most p-type in character indicating that at and near the surface the valence band is closer to the Fermi level. The HTSG sample's shape is a little different with a sharper drop off as it approaches zero. It is slightly less p-type in character. The sulfur annealed is the least ptype in character. Its shape is like the HTSG on its low binding energy side but at its high binding energy side appears that it might have another peak. The onset and cutoff of the UPS spectra can be used to determine the energy levels of valence band edge relevant to the Fermi level and the work function of the material. 


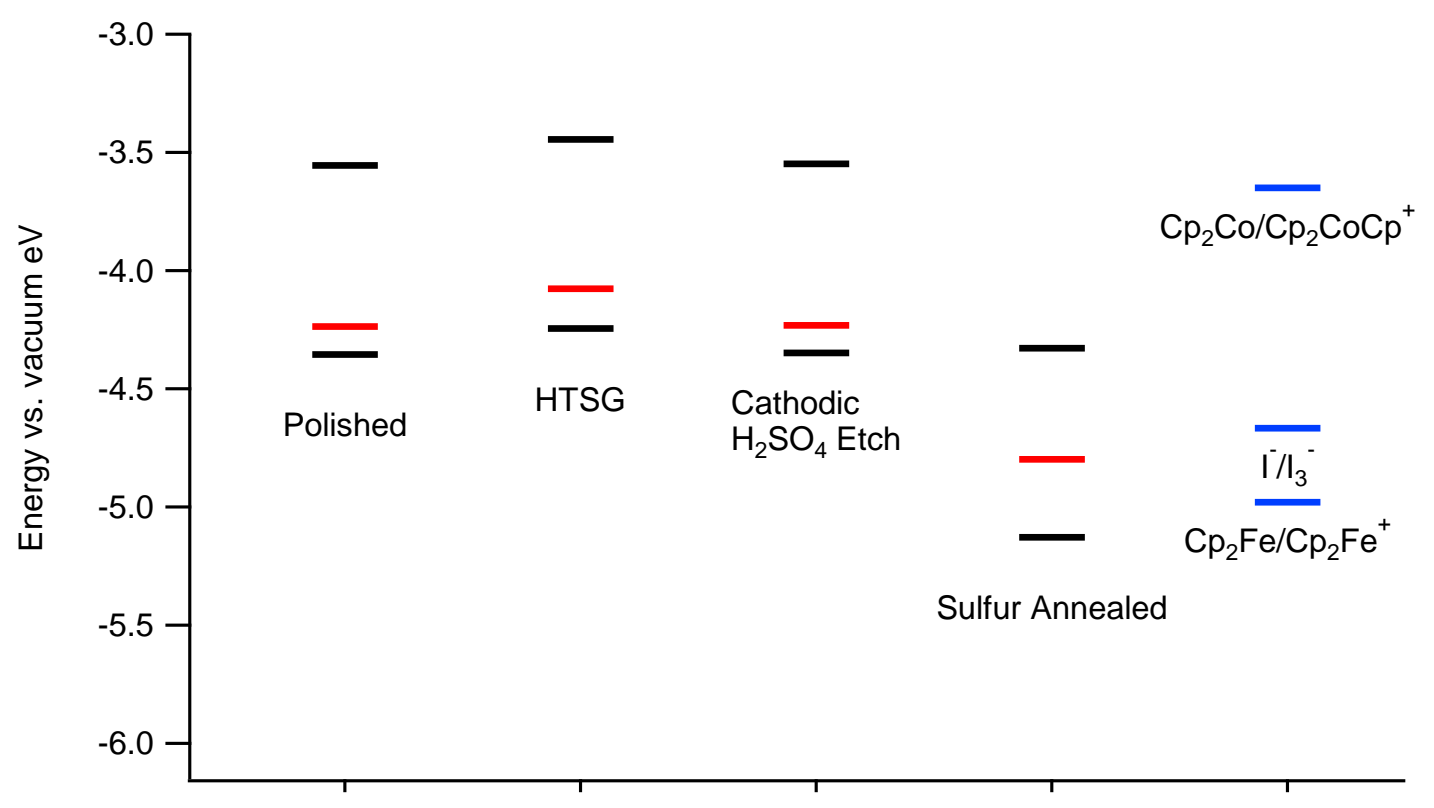

Figure 4.7. Band diagram for CVT samples with different histories generated from UPS spectra and assuming an electronic bandgap of $0.8 \mathrm{eV}$. The reduction potentials of the electrolyte solutions are included as well.

Figure 4.7 shows the band diagram for the different CVT sample histories. The onset was used to determine the distance between the valence band edge and the Fermi level and the cutoff was used to determine the work function. The reason for the difference in the work function is most likely due to the number of contaminants on the surface from handling in air which creates an interface dipole on the surface and decreases the work function. This is common for air exposed samples. Determining the work function of gold foil, a common reference material, sputtering with argon for 20 minutes was required before there was no longer a shift in the cutoff.

Fermi level pinning has been attributed for the low $V_{\mathrm{ph}}$ of pyrite. Pyrite is a bulk ntype material. It appears here that the Fermi level is pinned near the valence band edge. An increase in the separation between the two should signify a decrease in the pinning of the 
Fermi level at the surface. The cathodic etched and the polished have their Fermi level closest to their valence band edge, around $0.1 \mathrm{eV}$ apart. This should have the lowest photovoltage. The HTSG's separation is a little larger and the separation for the sulfur anneal is over $0.3 \mathrm{eV}$. This suggests that the photovoltage should be even higher than the others three. The only way to tell is by doing the actual photovoltage measurements.

\subsection{3 $V_{\mathrm{ph}}$ measurements of four different sample histories of CVT pyrite}

The electrochemical setup as described in section 2.3.6 was used to measure the photovoltage of the four samples of CVT pyrite with different surface treatment histories. Looking at Figure 4.7 a prediction can be made that based on the band positions the photovoltage of sulfur annealed CVT pyrite in the cobaltocene/cobaltocenium redox couple will be positive. The photovoltage for this electrode in the iodide/triiodide solution is expected to have a smaller amplitude and have positive values, while in the case of ferrocene/ferrocenium, smaller amplitudes but negative values are expected. These expectations are based on the relative positions of the energetics of pyrite ( $\mathbf{E}_{\mathrm{vbe}}, \mathbf{E}_{\mathrm{cbe}}$, and $\left.\mathbf{E}_{\mathrm{F}}\right)$ and the reduction potentials $\left(q E_{\mathrm{soln}}\right)$ of the redox couples. The results of the photovoltage measurements area shown in Table 4.2. 
Table 4.2. Photovoltage of Polished, HTSG, Etched, and sulfur annealed pyrite in 3 different electrolyte solutions at 1 sun and high intensity.

$$
\begin{aligned}
& \mathrm{V}_{\mathrm{ph}} \text { for pyrite/redox couple junction ( } \mathrm{mV} \text { vs. ) } \\
& \mathrm{Cp}_{2} \mathrm{Fe} / \mathrm{Cp}_{2} \mathrm{Fe}^{+} \quad \mathrm{Cp}_{2} \mathrm{Co} / \mathrm{Cp}_{2} \mathrm{Co}^{+} \quad \mathrm{I}^{-} / \mathrm{I}_{3}^{-}
\end{aligned}
$$

\begin{tabular}{cccccccc}
\hline Sample & Preparation & 1 sun & h. int. & 1 sun & h. int. & 1 sun h. int. \\
\hline CVT & Polished & 0 & 0 & 0 & 0 & 0 & 0 \\
\hline HTSG / CVT & as is & -4 & -24 & - & - & -5 & -30 \\
\hline CVT & etched & -56 & -117 & -40 & -106 & -50 & -110 \\
\hline CVT & $\begin{array}{c}\text { sulfur } \\
\text { annealed }\end{array}$ & -1 & -5 & -1 & -6.5 & -4 & -6 \\
\hline
\end{tabular}

Table 4.2 shows the measured photovoltages of the different sample history CVT pyrites slides in different electrolytes and at different light intensities. The polished CVT shows no photovoltage in any electrolyte or with any intensity of light. This could be from surface damaged incurred during the polishing steps. The HTSG/CVT shows a photovoltage, however for each electrolyte the photovoltage is negative. The measured photovoltages are also very close in value for each of the electrolytes. These electrolytes are far away enough in solution potential that this should not be the case. The surface of this material is not ideal in producing a photovoltage corresponding to a junction made of the surface of pyrite and the different solutions. The sulfur annealed CVT sample has shown little to no photovoltage values in all three electrolytes, and the values are also all negative which is like the other samples. The etched CVT pyrite shows the highest photovoltage. However, they are all similar in direction and in magnitude, which is not consistent with the reduction potentials of the three solutions as discussed above. It makes more sense that the photovoltage is being predetermined by a different junction, possibly present on the surface of pyrite before it is contacted by any of the redox solutions. 


\subsubsection{Relationship between measured $V_{\text {ph }}$ and the surface of CVT pyrite}

UPS characterization of all four pyrite samples suggested that the sulfur annealed pyrites should have the highest photovoltage values when contacted by $\mathrm{Cp}_{2} \mathrm{Co} / \mathrm{Cp}_{2} \mathrm{Co}^{+}$. This was not the case. The sample history with the highest by far photovoltage was after etching. How could this be?

It is important to understand what is happening on the surface of the material. It has been proposed that Fermi level pinning at the surface to the valence band edge is the cause of the low photovoltage. ${ }^{18}$ This creates a thin inversion layer, inverting from an n-type bulk to a p-type surface. This results in non-uniform depletion layer with very steep band bending near the surface. In this layer of steep band bending electrons can tunnel across diminishing the photovoltage. This results in limiting the photovoltage to the bending of the depletion layer outside of the steep band bent surface which has been estimated to be around $180 \mathrm{mV} .^{1}$

The sulfur anneal has been shown to improve the near surface crystallinity, however this sample produced negligible photovoltages. It is possible that it has not completely improved the actual surface layer in which case presence of an inversion layer on the surface is expected. If the near surface was repaired than this inversion layer is even smaller, making it even more likely that electrons will tunnel across it. On the other hand, acid etched surfaces of pyrite have never produced photovoltages over $200 \mathrm{mV}$ in the literature, which is also consistent with the earlier described Fermi level pinning. However, as it was discussed above, reducing the carrier tunneling increases the photovoltage. Perhaps the etching of the pyrite surface creates a thicker inversion layer limiting the 
tunneling. This is consistent with relatively high photovoltages obtained after sulfuric acid etching but lower $V_{\mathrm{ph}}$ values of the sulfur annealed CVT pyrite.

\subsection{Conclusions}

The surface of pyrite was successfully modified with sulfur annealing improving its crystallinity. The sulfur annealing process, high temperature solution growth, or mechanical polishing steps did not improve the photovoltage of CVT pyrite. The only improvement was through the cathodic etch of pyrite in sulfuric acid. The improvement may be the result of the increased thickness of the surface defect layer which lengthens the inversion layer, limits the tunneling of electrons across the boundary so that the photovoltage increases, is predefined by the inversion layer and is independent of energetics of the outside contact or junction as it was observed. Sulfur annealing was shown to improve the near surface layer but according to electrochemical studies did not remove of all the surface defects so that the inversion layer was absent. 


\section{Summary and future}

The pyrite used in this research has been fabricated in different ways. First the conversion of iron pentacarbonyl and sulfur into pyrite in an organic solvent, or the repeated sulfur annealing of iron into pyrite were used as feedstock for growth of larger crystals. The larger crystals were grown by; (1) a HTSG onto natural pyrite, (2) CVT growth of pyrite with iron(II) bromide as a transport agent into single crystal or (3) as epitaxial growths onto natural pyrite, and (4) HTSG onto CVT pyrite that had been removed from the natural pyrite which it had used as a substrate.

The photovoltaic properties of the pyrite were dependent on the substrates used for growth. Improvements to photovoltage values of pyrite devices were obtained through replacement of the natural substrate with the CVT substrate.

The photovoltage of the substrates were improved by a cathodic etch of the surface. The photovoltage was not improved by sulfur annealing the surface of the pyrite, however this step was shown to improve the surface crystallinity. It is possible that the improved photovoltage is because of the increased thickness of the surface layer resulting in a photovoltage that is a result of the junction of the surface layer and the bulk material where the sulfur annealed pyrite's surface layer creates the same junction although the thickness of the surface layer is much thinner, so much so that electrons can tunnel through so that there is no separation of charge carriers which results in no photovoltage.

It was successfully demonstrated that synthetic pyrite results in higher photovoltage values than those measured for the natural pyrite. Synthetic pyrite also has the advantage of repeatability. The inconsistencies of natural pyrite make it hard to study its 
photoelectrochemical properties consistently. Now that CVT substrates provide a consistent substrate for HTSG of pyrite, HTSG itself can be optimized and studied. Ideally the HTSG onto CVT pyrite could be improved to a degree which allows for the removal of the CVT substrate so that the properties of standalone HTSG can be determined. This researched has shown that the photovoltage is dependent on the method of growth. Isolating HTSG pyrite is imperative to determine its photovoltage.

Surface treatments improve the photovoltage of pyrite. The cause of this improvement needs to be determined. This research suggests that the cause may be the increased thickness of the surface layer. A more thorough investigation involves the optimization of the etching for a correlation of etching parameters to photovoltage, and the surface layer. The etching still done in sulfuric acid but varying the electrochemical potential at which it is held to thermodynamically control the reactions on the surface. The photovoltage measured as described in this document and the surface layer monitored with photoelectron spectroscopy with the high sensitivity of angle resolved and the ability to tune the beam with a synchrotron source. This will provide the most surface sensitive information.

Attempts can also be made to functionalize the surface of pyrite to increase the photovoltage by replacing defects on the surface with a new chemical identity. This is thought to remove the surface defects, eliminating unwanted energy levels that they create within the bandgap. The photovoltage can be measured in an electrochemical cell and high sensitivity angle resolved synchrotron photoelectron spectroscopy can be used to determine the identity and binding energies of the surface molecules. 
Pyrite has been doped to change its $p$ - and $n$-type character. The technique of using the CVT growth in this research, with a natural substrate to grow off of and then removing it, leaving a standalone synthetically grown CVT crystal, can be further modified. It could be thinned and then doped on either side by thermal diffusion of dopant elements. Another route for doping would involve doping during the CVT growth process. Either way a $p-n$ junction should be able to be made and allow for optimizing and full use of the pyrite's bandgap. This is another step forward that could be taken for pyrite research and is believed to move around the surface problems of pyrite. This may be a difficult process as pyrite is very absorbing of light. The geometry of this junction would have to be optimized for the optimal charge separation by light reaching the depletion region at the junction to optimize the photovoltage. 


\section{References}

1. Limpinsel, M.; Farhi, N.; Berry, N.; Lindemuth, J.; Perkins, C. L.; Lin, Q.; Law, M., An inversion layer at the surface of n-type iron pyrite. Energy Environ. Sci. 2014, 7 (6), 1974-1989.

2. Shockley, W.; Queisser, H. J., Detailed Balance Limit of Efficiency of p-n Junction Solar Cells. J. Appl. Phys. 1961, 32 (3), 510-519.

3. Ennaoui, A.; Fiechter, S.; Goslowsky, H.; Tributsch, H., Photoactive Synthetic Polycrystalline Pyrite (FeS2). J. Electrochem. Soc. 1985, 132 (7), 1579-1582.

4. Ennaoui, A.; Fiechter, S.; Pettenkofer, C.; Alonso-Vante, N.; Buker, K.; Bronold, M.; Hopfner, C.; Tributsch, H., Iron disulfide for solar energy conversion. Sol. Energy Mater. Sol. Cells 1993, 29, 289-370.

5. Ennaoui, A.; Fiechter, S.; Jaegermann, W.; Tributsch, H., Photoelectrochemistry of Highly Quantum Efficient Single-Crystalline n-FeS2 (Pyrite). J. Electrochem. Soc. 1986, 133 (1), 97-106.

6. Ennaoui, A.; Tributsch, H., Iron sulphide solar cells. Sol. Cells 1984, 13, 197-200.

7. Ennaoui, A.; Tributsch, H., Light-Induced Electron Transfer and Photoelectrocatalysis of Chlorine Evolution at FeS2 Electrodes. $J$. Electroanalitical Chem. 1986, 204, 185-195.

8. John, E., Nature's Building Blocks; Oxford University Press: New York, 2011.

9. Mineral Commodity Summaries 2015; U.S. Geological Survey, 2015.

10. Wadia, C.; Alivisatos, A. P.; Kammen, D. M., Materials Availability Expands the Opportunity for Large-Scale Photovoltaics Deployment. Environ. Sci. Technol. 2009, 43 (6), 2072-2077.

11. Polman, A.; Atwater, H. A., Photonic design principles for ultrahigh-efficiency photovoltaics. Nat. Mater. 2012, 11 (3), 174-177.

12. Rau, U.; Kirchartz, T., On the thermodynamics of light trapping in solar cells. Nat. Mater. 2014, 13 (2), 103-104.

13. Atwater, H. a; Polman, A., Reply to "On the thermodynamics of light trapping in solar cells". Nat. Mater. 2014, 13 (2), 104-105.

14. Ennaoui, A.; Fiechter, S.; Tributsch, H.; Giersig, M.; Vogel, R.; Weller, H., Photoelectrochemical Energy Conversion Obtained with Ultrathin OrganoMetallic-Chemical-Vapor-Deposition Layer of FeS2 (Pyrite) on TiO2. J. Electrochem. Soc. 1992, 139 (9), 2514-2518.

15. Buker, K.; Alonso-Vante, N.; Tributsch, H., Photovoltaic output limitation of nFeS2 (pyrite) Schottky barriers: A temperature-dependent characterization. $J$. Appl. Phys. 1992, 72 (12), 5721-5728.

16. Sayigh, A. A. M., Energy and the Environment into the 1990s; 1990.

17. Mishra, K. K.; Osseo-Asare, K., Fermi level pinning at pyrite (FeS2)/electrolyte junctions. J. Electrochem. Soc. 1992, 139 (3), 7-10.

18. Bronold, M.; Tomm, Y.; Jaegermann, W., Surface states on cubic d-band semiconductor pyrite (FeS2). Surf. Sci. 1994, 314, L931-L936.

19. Bronold, M.; Pettenkofer, C.; Jaegermann, W., Surface photovoltage measurements on pyrite (100) cleavage planes: Evidence for electronic bulk defects. J. Appl. ... 1994, 76 (10), 5800-5808. 
20. Guanzhou, Q.; Qi, X.; Yuehua, H., First-principles calculation of the electronic structure of the stoichiometric pyrite FeS2(100) surface (No. 03-11). Comput.

Mater. Sci. 2004, 29 (1), 89-94.

21. Herbert, F. W.; Krishnamoorthy, A.; Van Vliet, K. J.; Yildiz, B., Quantification of electronic band gap and surface states on FeS2(100). Surf. Sci. 2013, 618, 53-61.

22. Liang, D.; Cabán-Acevedo, M.; Kaiser, N. S.; Jin, S., Gated Hall Effect of Nanoplate Devices Reveals Surface-State-Induced Surface Inversion in Iron Pyrite Semiconductor. Nano Lett. 2014, 14, 6754-6760.

23. Buker, K.; Alonso-Vante, N.; Scheer, R.; Tributsch, H., Influence of Electrochemical Activation and Surface Orientation on the Photoresponse of Single Crystalline Pyrite/Electrolyte and Pyrite/Metal Junctions. Ber. Bunsenges. Phys. Chem. 1994, 98 (5), 674-682.

24. Hu, J.; Zhang, Y.; Law, M.; Wu, R., First-principles studies of the electronic properties of native and substitutional anionic defects in bulk iron pyrite. Phys. Rev. B 2012, 85 (8), 85203.

25. Wei, D.; Osseo-Asare, K., Semiconductor electrochemistry of particulate pyrite: dissolution via hole and electron pathways. J. Electrochem. Soc. 1996, 143 (10), 3192-3198.

26. Lalvani, S. B.; Deneve, B. A.; Weston, A., Passivation of pyrite due to surface treatment. Fuel 1990, 69, 1567-1569.

27. Li, X.-P.; Alsonso Vante, N.; Tributsch, H., Involvement of coordination chemistry during electron transfer in the stabilization of the pyrite $(\mathrm{FeS} 2)$ photoanode. J. Electroanal. Chem 1988, 242, 255-264.

28. Schubert, B.; Tributsch, H., Photoinduced Electron Transfer by Coordination Chemical Pathways across Pyrite/Electrolyte Interfaces. Inorg. Chem. 1990, 29, 5041-5046.

29. Sasaki, K.; Tsunekawa, M.; Hasebe, K.; Konno, H., Effect of anionic ligands on the reactivity of pyrite with Fe (III) ions in acid solutions. Colloids Surfaces A ... 1995, 101, 39-49.

30. Cabán-Acevedo, M.; Kaiser, N. S.; English, C. R.; Liang, D.; Thompson, B. J.; Chen, H.; Czech, K. J.; Wright, J. C.; Hamers, R. J.; Jin, S., Ionization of HighDensity Deep Donor Defect States Explains the Low Photovoltage of Iron Pyrite Single Crystals. J. Am. Chem. Soc. 2014, 136 (49), 17163-17179.

31. Hung, A.; Muscat, J.; Yarovsky, I.; Russo, S. P., Density-functional theory studies of pyrite FeS2(100) and (110) surfaces. Surf. Sci. 2002, 513, 511-524.

32. Zhang, Y. N.; Hu, J.; Law, M.; Wu, R. Q., Effect of surface stoichiometry on the band gap of the pyrite FeS2 (100) surface. Phys. Rev. B 2012, 85 (8), 085314(1-5).

33. Yakovkin, I. N.; Petrova, N. V., Influence of the thickness and surface composition on the electronic structure of FeS2 layers. Appl. Surf. Sci. 2016, 377, 184-190.

34. Alonso-Vante, N.; Chatzitheodorou, G.; Fiechter, S.; Mgoduka, N.; Poulios, I.; Tributsch, H., Interfacial Behavior of Hydrogen-Treated Sulphur Deficient Pyrite (FeS2-x). Sol. Energy Mater. 1988, 18, 9-21.

35. Ennaoui, A.; Fiechter, S.; Mai, J.; Szacki, W., Chemical Vapour Transport of 
Pyrite (FeS2) with Halogen (Cl, Br ,I). J. Cryst. Growth 1986, 78, 438-444.

36. Mochizuki, K.; Iwanaga, H., Vapor growth and polarity in ZnS crystals. Journal of Crystal Growth. 1985, pp 459-462.

37. Fleming, J. G., Growth of FeS2 (Pyrite) From Te Melts. J. Cryst. Growth 1988 , 92, 287-293.

38. Schieck, R.; Hartmann, A.; Fiechter, S.; Konenkamp, R.; Wetzel, H., Electrical properties of natural and synthetic pyrite (FeS2 ) crystals. J. Mater. Res. 1990, 5 (7), 1567-1572.

39. Chase, M. W., JANAF Thermochemical Tables, 3rd ed.; American Institute of Physics for the National Bureau of Standards: New York, 1986.

40. Ipser, H.; Komarek, K. L., Thermodynamic properties of Iron-Tellurium alloys. Monatshefte fur Chemie 1974, 105, 1344-1361.

41. Siebert, D.; Miller, R.; Fiechter, S.; Dulski, P.; Hartmann, A., An EPR investigation of $\mathrm{Cr} 3+$ and $\mathrm{Ni2}+$ in synthetic pyrite crystals. Zeitschrift Fur Naturforsch. Sect. A-A J. Phys. Sci. 1990, 45 (11-12), 1267-1272.

42. Mrowec, S.; Przybylski, K., Defect and Transport Properties of Sulfides and Sulfidation Metals. High Temp. Mater. Process. 1984, 6 (1-2), 1-80.

43. Chatzitheodorou, G.; Fiechter, S.; Kunst, M.; Luck, J.; Tributsch, H., Low Temperature Chemical Preparation of Semiconducting Transition Metal Chalcogenide Films for Energy Conversion and Storage, Lubrication and Surface Protection. Mat. Res. Bull. 1988, 23 (9), 1261-1271.

44. Meng, L.; Liu, M. S., Thin pyrite ( FeS 2 ) films prepared by thermal-sulfurating iron films at various temperatures. 1999, 60, 168-172.

45. Meng, L.; Liu, Y. H.; Huang, W., Synthesis of pyrite thin films obtained by thermal-sulfurating iron films at different sulfur atmosphere pressure. Mater. Sci. Eng. B 2002, 90 (1-2), 84-89.

46. Wan, D.; Wang, B.; Wang, Y.; Sun, H.; Zhang, R.; Wei, L., Effects of the sulfur pressure on pyrite FeS2 films prepared by sulfurizing thermally iron films. $J$. Cryst. Growth 2003, 257 (3-4), 286-292.

47. Wan, D.; Wang, Y.; Wang, B.; Ma, C.; Sun, H.; Wei, L., Effects of the crystal structure on electrical and optical properties of pyrite FeS2 films prepared by thermally sulfurizing iron films. J. Cryst. Growth 2003, 253 (1-4), 230-238.

48. Meng, L.; Liu, Y. H.; Tian, L., Structural, optical and electrical properties of polycrystalline pyrite $(\mathrm{FeS} 2)$ films obtained by thermal sulfuration of iron films. $J$. Cryst. Growth 2003, 253 (1-4), 530-538.

49. Meng, L.; Liu, Y. H.; Tian, L., Evolutions of structure, composition and optical absorption behavior of pyrite films formed by sulfurating iron. Mater. Res. Bull. 2003, 38 (6), 941-948.

50. Wan, D.; Wang, B.; Zhou, C.; Ma, C.; Wang, Y.; Zhang, R.; Wei, L., Positron annihilation study of defects in pyrite FeS2 films prepared by sulfurizing thermally iron films. Phys. B Condens. Matter 2004, 344 (1-4), 489-494.

51. Wan, D.; He, Q.; Zhang, L.; Jia, Q.; Zhang, R.; Zhang, H.; Wang, B.; Wei, L., Study on pyrite FeS2 films deposited on $\mathrm{Si}(100)$ substrate by synchrotron radiation surface X-ray diffraction method. J. Cryst. Growth 2004, 268 (1-2), 222-226. 
52. Zhang, H.; Wang, B.; Zhang, Z.; Wang, P.; Wei, L.; Jia, Q.; Wang, Y., Experimental Studies on FeS2 Films Prepared on Si ( 100 ) Substrates by Synchrotron Radiation Surface X-Ray Diffraction Method *. High Energy Phys. Nucl. Phys. 2005, 29, 32-36.

53. Li, X. F.; Wang, Y.; Meng, L., Microstructure and optical absorption of FeS2 films formed by sulfurizing precursive iron of various crystallite scales. Mater. Res. Bull. 2009, 44 (2), 462-467.

54. Huang, L.; Liu, Y.; Meng, L., Pyrite Films Grown by Sulfurizing Precursive Iron of Different Crystallizing Status. J. Mater. Sci. Technol. 2009, 25 (2), 237-241.

55. Huang, L. Y.; Meng, L., Crystallographic behavior of FeS2 films formed on different substrates. Mater. Chem. Phys. 2010, 124 (1), 413-416.

56. de las Heras, C.; Sanchez, C., Characterization of iron pyrite thin films obtained by flash evaporation. Thin Solid Films 1991, 199, 259-267.

57. Ferrer, I. J.; de las Heras, C.; Sanchez, C., Physical properties of Cu-doped FeS2 Pyrite thin films. Appl. Surf. Sci. 1993, 70/71, 588-592.

58. Ferrer, I. J.; Caballero, F.; de las Heras, C.; Sanchez, C., Preparation of n-type doped FeS2 thin films. Solid State Commun. 1994, 89 (4), 349-352.

59. Ferrer, I. J.; de las Heras, C.; Sanchez, C., The effect of Ni impurities on some structural properties of pyrite thin films. J. Phys. ... 1995, 2115, 2115-2121.

60. Ares, J. R.; Leon, M.; Arozamena, N. M.; Sanchez-Paramo, J.; Celis, P.; Ferrer, I. J.; Sanchez, C., Evolution of the Seebeck coefficient during the formation and crystallization of pyrite thin films. J. Phys. Condens. Matter 1998, 10, 4281-4289.

61. Ares, J. R.; Ferrer, I. J.; Cuevas, F.; Sanchez, C., Growth of pyrite thin-films investigated by thermoelectric measurements. Thin Solid Films 2001, 387, 97-99.

62. Ares, J. R.; Ferrer, I. J.; Sanchez, C., Majority carriers in pyrite thin films: an analysis based on Seebeck and Hall coefficient measurements. Thin Solid Films 2003, 431-432, 511-513.

63. Ferrer, I. J.; de las Heras, C.; Menendez, N.; Tornero, J.; Sanchez, C., Application of Mossbauer spectroscopy to study the formation of iron pyrite thin films. $J$. Mater. Sci. 1993, 28, 389-393.

64. De Las Heras, C.; Martín De Vidales, J. L.; Ferrer, I. J.; Sánchez, C., Structural and microstructural features of pyrite FeS2-x thin films obtained by thermal sulfuration of iron. J. Mater. Res. 1996, 11, 211-220.

65. de las Heras, C.; Lifante, G., Optical parameters of pyrite thin films. J. Appl. Phys. 1997, 82 (10), 5132.

66. Pascual, A.; Ares, J. R.; Ferrer, I. J.; Sánchez, C., Electrical resistance evolution of Fe thin films during their sulphuration process. Appl. Surf. Sci. 2004, 234 (1-4), 355-361.

67. Ares, J. R.; Pascual, A.; Ferrer, I. J.; Sanchez, C., Lattice intrinsic defects and electrical resistivity in pyrite thin films. Thin Solid Films 2004, 451-452, 233-236.

68. Ares, J. R.; Pascual, A.; Ferrer, I. J.; Sanchez, C., Grain and crystallite size in polycrystalline pyrite thin films. Thin Solid Films 2005, 480-481, 477-481.

69. Diaz-Chao, P.; Ferrer, I. J.; Sanchez, C., Co distribution through n-type pyrite thin films. Thin Solid Films 2008, 516, 7116-7119. 
70. Díaz-Chao, P.; Ares, J. R.; Ferrer, I. J.; Sánchez, C., Role of cation diffusion in the formation mechanism and properties of cobalt-doped n-type pyrite thin films. $J$. Mater. Sci. 2013, 48 (14), 4914-4924.

71. Vahidi, M.; Lehner, S. W.; Buseck, P. R.; Newman, N., Growth of epitaxial pyrite (FeS2) thin films using sequential evaporation. Acta Mater. 2013, 61 (19), 73927398.

72. Chatzitheodorou, G.; Fiechter, S.; Konenkamp, R.; Kunst, M.; Jaegermann, W.; Tributsch, H., Thin photoactive FeS2 (pyrite) films. Mater. Res. ... 1986, 21 (c), 1481-1487.

73. Smestad, G.; Da Silva, A.; Tributsch, H.; Fiechter, S.; Kunst, M.; Meziani, N.; Birkholz, M., Formation of semiconducting iron pyrite by spray pyrolysis. Sol. energy ... 1989, 18, 299-313.

74. Smestad, G.; Ennaoui, A.; Fiechter, S.; Tributsch, H.; Hofmann, W. K.; Birkholz, M., Photoactive Thin Film Semiconducting Iron Pyrite Prepared by Sulfurization of Iron Oxides. Sol. Energy Mater. 1990, 20, 149-165.

75. Willeke, G.; Dasbach, R.; Sailer, B.; Bucher, E., Thin pyrite (FeS2) films prepared by magnetron sputtering. Thin Solid Films 1992, 213 (2), 271-276.

76. de las Heras, C.; Ferrer, I. J.; Sanchez, C.; Sánchez, C., Pyrite thin films : Improvements in their optical and electrical properties by annealing at different temperatures in a sulfur atmosphere. J. Appl. Phys. 1993, 74 (7), 4551-4556.

77. Lichtenberger, D.; Ellmer, K.; Schieck, R.; Fiechter, S.; Tributsch, H., Structural, optical and electrical properties of polycrystalline iron pyrite layers deposited by reactive d.c. magnetron sputtering. Thin Solid Films 1994, 246 (1-2), 6-12.

78. de las Heras, C.; Bausa, L. E., NdS 2 formation in Nd-doped pyrite films. J. Phys. Condens. Matter 1997, 9, 9483-9495.

79. Seefeld, S.; Limpinsel, M.; Liu, Y.; Farhi, N.; Weber, A.; Zhang, Y.; Berry, N.; Kwon, Y. J.; Perkins, C. L.; Hemminger, J. C.; et al., Iron Pyrite Thin Films Synthesized from an Fe(acac)3 Ink. J. Am. Chem. Soc. 2013, 135 (11), 4412-4424.

80. Oertel, J.; Ellmer, K.; Bohne, W.; Rohrich, J.; Tributsch, H., Growth of n-type polycrystalline pyrite $(\mathrm{FeS} 2)$ films by metalorganic chemical vapour deposition and their electrical characterization. J. Cryst. Growth 1999, 199, 1205-1210.

81. Gomes, A.; Ares, J. R.; Ferrer, I. J.; da Silva Pereira, M. I.; Sanchez, C., Formation of n-type pyrite films from electrodeposited iron sulphides: effect of annealing temperature. Mater. Res. Bull. 2003, 38 (7), 1123-1133.

82. Berry, N.; Cheng, M.; Perkins, C. L.; Limpinsel, M.; Hemminger, J. C.; Law, M., Atmospheric-Pressure Chemical Vapor Deposition of Iron Pyrite Thin Films. Adv. Energy Mater. 2012, 2 (9), 1124-1135.

83. Echarri, A.; Sanchez, C., "n" type semiconductivity in natural single crystals of FeS 2(pyrite). Solid State Commun. 1974, 15, 827-831.

84. Jaegermann, W.; Tributsch, H., Photoelectrochemical reactions of FeS2 (pyrite) with $\mathrm{H} 2 \mathrm{O}$ and reducing agents. J. Appl. Electrochem. 1983, 13, 743-750.

85. Ennaoui, A.; Tributsch, H., Energetic Characterization of the Photoactive FeS2 (Pyrite) Interface. Sol. Energy Mater. 1986, 14, 461-474.

86. Nevskaia, D. M.; Ferrer, I. J.; Sanchez, C., Open circuit photopotentials in n-FeS 2 
natural single-crystal / aqueous electrolyte junctions. Sol. Energy 1991, 22, $127-$ 135.

87. Salvador, P.; Tafalla, D.; Tributsch, H.; Wetzel, H., Reaction Mechanisms at the nFeS2 / I Interface. 1991, 138 (11).

88. Ferrer, I. J.; Sanchez, C., Photoelectrochemical response and optical absorption of pyrite (FeS 2) natural single crystals. Solid State Commun. 1992, 81 (5), 371-374.

89. de las Heras, C.; Salto, D.; Ferrer, I. J.; Sanchez, C., In situ electrical resistivity measurements during the sulphuration of pyrite and Fe thin films. J. Phys. Condens. Matter 1994, 6, 899-906.

90. Tomm, Y.; Schieck, R.; Ellmer, K.; Fiechter, S., Growth mechanism and electronic properties of doped pyrite (FeS2) crystals. J. Cryst. Growth 1995, 146, 271-276.

91. Morsli, M.; Bonnet, A.; Cattin, L.; Conan, A.; Fiechter, S., Electrical Properties of a Synthetic Pyrite FeS2 Non Stoichiometric Crystal. J. Phys. I 1995, 5 (6), 699705.

92. Büker, K.; Fiechter, S.; Eyert, V.; Tributsch, H., Photoelectrochemistry of Highly Zn-Doped Pyrite as Compared with Isostructural FeS2. J. Electrochem. Soc. 1999, 146 (1), 261-265.

93. Thomas, B.; Ellmer, K.; Bohne, W.; Rohrich, J.; Kunst, M.; Tributsch, H., Photoeffects in cobalt doped pyrite (FeS 2) films. Solid state ... 1999, 111, 235240.

94. Abd El Halim, A. M.; Fiechter, S.; Tributsch, H., Control of interfacial barriers in n-type FeS2 (pyrite) by electrodepositing metals $(\mathrm{Co}, \mathrm{Cu})$ forming isostructural disulfides. Electrochim. Acta 2002, 47 (16), 2615-2623.

95. Tributsch, H.; Fiechter, S.; Jokisch, D.; Rojas-Chapana, J.; Ellmer, K., Photoelectrochemical Power, Chemical Energy and Catalytic Activity for Organic Evolution on Natural Pyrite Interfaces. Orig. Life Evol. Biosph. 2003, 32, 129-162.

96. Ho, C. H.; Huang, C. E.; Wu, C. C., Preparation and characterization of Niincorporated FeS2 single crystals. J. Cryst. Growth 2004, 270 (3-4), 535-541.

97. Blenk, O.; Bucher, E.; Willeke, G., P-Type Conduction in Pyrite Single Crystals Prepared By Chemical Vapor Transport. Appl. Phys. Lett. 1993, 62 (17), 2093.

98. Lehner, S. W.; Savage, K. S.; Ayers, J. C., Vapor growth and characterization of pyrite $(\mathrm{FeS} 2)$ doped with $\mathrm{Co}, \mathrm{Ni}$, and $\mathrm{As}$ : Variations in semiconducting properties. J. Cryst. Growth 2005, 286 (2), 306-317.

99. Ho, C. H.; Huang, Y. S.; Tiong, K. K., Characterization of near band-edge properties of synthetic p-FeS2 iron pyrite from electrical and photoconductivity measurements. J. Alloys Compd. 2006, 422 (1-2), 321-327.

100. Lehner, S. W.; Newman, N.; van Schilfgaarde, M.; Bandyopadhyay, S.; Savage, K.; Buseck, P. R., Defect energy levels and electronic behavior of Ni-, Co-, and As-doped synthetic pyrite (FeS2). J. Appl. Phys. 2012, 111 (8), 83717.

101. Webber, D. H.; Buckley, J. J.; Antunez, P. D.; Brutchey, R. L., Facile dissolution of selenium and tellurium in a thiol-amine solvent mixture under ambient conditions. Chem. Sci. 2014, 5 (6), 2498.

102. Kurtin, S.; McGill, T. C.; Mead, C. a., Fundamental transition in the electronic nature of solids. Phys. Rev. Lett. 1969, 22 (26), 1433-1436. 
103. Bronold, M.; Büker, K.; Kubala, S.; Pettenkofer, C.; Tributsch, H., Surface Preparation of $\mathrm{FeS} 2$ via electrochemical etching and interface formation with metals. ... status solidi 1993, 135, 231-243.

104. Lewis, N. S., A Quantitative Investigation of the Open-Circuit Photovoltage at the Semiconductor/Liquid Interface. J. Electrochem. Soc. 1984, 131 (11), 2496.

105. Velásquez, P.; Leinen, D.; Pascual, J.; Ramos-Barrado, J. R.; Grez, P.; Gómez, H.; Schrebler, R.; Del Río, R.; Córdova, R., A chemical, morphological, and electrochemical (XPS, SEM/EDX, CV, and EIS) analysis of electrochemically modified electrode surfaces of natural chalcopyrite $(\mathrm{CuFeS} 2)$ and pyrite $(\mathrm{FeS} 2)$ in alkaline solutions. J. Phys. Chem. B 2005, 109 (11), 4977-4988.

106. Tong, Q.; Johansson, E., Photoelectrochemical Behavior of FeS2 in Aqueous and Nonaqeuous I-/I3- Electrolytes. J. Electrochem. Soc. 2014, 161 (14), H950H954.

107. Dohrmann, J. K.; Chuan, M.; Schaaf, N.-S., Photocalorimetry at semiconductor electrodes: theory, technique and applications. Sol. Energy Mater. Sol. Cells 1996, 43 (3), 273-296.

108. Samad, L.; Cabán-Acevedo, M.; Shearer, M. J.; Park, K.; Hamers, R. J.; Jin, S., Direct Chemical Vapor Deposition Synthesis of Phase-Pure Iron Pyrite (FeS2) Thin Films. Chem. Mater. 2015, 150408161512006.

109. Schaaf, N.-S.; Dohrmann, J. K.; Seeliger, W.; Tributsch, H., Photoanodic Reactions at Natural Pyrite ( 100 ) Studied by Photocalorimetry : Internal Quantum and Energy Efficiencies and Peltier Heats after Different Surface Treatment. 1996, 107.

110. Büker, K.; Alonso-Vante, N.; Tributsch, H., Photoelectrochemical Investigations of Complex Formation Phenomena on Oriented n-Pyrite (FeS2) Crystal Surfaces. Ber. Bunsenges. Phys. Chem. 1996, 100 (11), 1808-1813.

111. Bungs, M.; Tributsch, H., Electrochemical and Photoelectrochemical Insertion and Transport of Hydrogen in Pyrite. Phys Chem 1997, No. 12, 1844-1850.

112. Herbert, F. W.; Krishnamoorthy, A.; Ma, W.; Van Vliet, K. J.; Yildiz, B., Dynamics of point defect formation, clustering and pit initiation on the pyrite surface. Electrochim. Acta 2014, 127, 416-426.

113. Robert, V.; Beitriige, R.; Meisel, V. K., Das Zustandsdiagramm Pyrit, Magnetkies , Troilit und Schwefeldampf, beurteilt nach Schwefeldampfdrucjen, Rontgenbildern, Dichten und magnetischen Messungen. Z. Anorg. u. allg. Chem, 1932, 205, 273-286.

114. Ennaoui, A.; Schroetter, S.; Fiechter, S.; Tributsch, H., Infrared spectroscopic and X-ray diffraction characterization of iron disulphide thin films prepared by metalorganic chemical vapour deposition. J. Mater. Sci. Lett. 1992, 11, 1131-1133.

115. Tong, Q.; Young, E. R.; Johansson, E., Infrared Reflection-Absorption Spectroscopy of an Isotropic Adsorbate Layer on Pyrite. J. Phys. Chem. C 2013, 117, 25982-25990.

116. Steinhagen, C.; Harvey, T. B.; Stolle, C. J.; Harris, J.; Korgel, B. a., Pyrite Nanocrystal Solar Cells: Promising, or Fool's Gold? J. Phys. Chem. Lett. 2012, 3 (17), 2352-2356. 
117. Li, Y.; Han, Z.; Jiang, L.; Su, Z.; Liu, F.; Lai, Y.; Liu, Y., Template-directed synthesis of ordered iron pyrite (FeS2) nanowires and nanotubes arrays. J. Sol-Gel Sci. Technol. 2014, 72 (1), 100-105.

118. Jiang, F.; Peckler, L. T.; Muscat, A. J., Phase Pure Pyrite FeS 2 Nanocubes Synthesized Using Oleylamine as Ligand, Solvent, and Reductant. 2015.

119. Cabán-Acevedo, M.; Liang, D.; Chew, K.; Jin, S., Synthesis, Characterization, and Variable Range Hopping Transport of Pyrite (FeS2) Nanorods, Nanobelts, and Nanoplates. $A C S$... 2013, No. 2, 1731-1739.

120. Morrish, R.; Silverstein, R.; Wolden, C. A., Synthesis of stoichiometric FeS2 through plasma-assisted sulfurization of Fe2O3 nanorods. J. Am. Chem. Soc. 2012, 134 (43), 17854-17857.

121. Vogt, H.; Chattopadhyay, T.; Stolz, H. J., Complete first-order Raman spectra of the pyrite structure compounds FeS2, MnS2 AND SiP2. J. Phys. Chem. Solids 1983, 44 (9), 869-873.

122. Sourisseau, C.; Fouassier, M., The Vibrational Properties and Valence Force Fields of FeS2, RuS2 Pyrites and FeS2 Marcasite. 1991, 52 (3).

123. Fiechter, S.; Birkholz, M.; Hartmann, A.; Dulski, P.; Giersig, M.; Tributsch, H., The microstructure and stoichiometry of pyrite FeS2- x. J. Mater. Res. 1992, 7 (7), 1829-1838.

124. Gil-Lozano, C.; Losa-Adams, E.; Dávila, A. F.; Gago-Duport, L., Pyrite nanoparticles as a fenton-like reagent for in situ remediation of organic pollutants. Beilstein J. Nanotechnol. 2014, 5 (1), 855-864.

125. Hsiao, S.; Wu, K.; Huang, S.; Liu, S.; Chiu, S.; Chou, L., FeS2 ultrathin films prepared using well-dispersed pyrite nanocrystal inks. 2015, 15201, 0-4.

126. Watson, E. B.; Cherniak, D. J.; Frank, E. A., Retention of biosignatures and massindependent fractionations in pyrite: Self-diffusion of sulfur. Geochim. Cosmochim. Acta 2009, 73 (16), 4792-4802.

127. Savage, K. S.; Stefan, D.; Lehner, S. W., Impurities and heterogeneity in pyrite: Influences on electrical properties and oxidation products. Appl. Geochemistry 2008, 23 (2), 103-120.

128. Bouchard, R. J., The Preparation of Single Crystals of FeS2 CoS2 and NiS2 Pyrites by Chlorine Transport. J. Cryst. Growth 1968, 2, 40-44.

129. Bither, T. A.; Bouchard, R. J.; Cloud, W. H.; Donohue, P. C.; Siemons, W. J., Transition metal pyrite dichalcogenides. High-pressure synthesis and correlation of properties. Inorg. Chem. 1968, 7 (11), 2208-2220.

130. Yamada, S.; Nanjo, J.; Nomura, S.; Hara, S., Morphology of iron pyrite crystals. J. Cryst. Growth 1979, 46 (1), 10-14.

131. Chongyang, L.; Pettenkofer, C.; Tributsch, H., Enhancement of photoactivity in pyrite (FeS2) interfaces by photoelectrochemical processes. Surf. Sci. 1988, 204 (3), 537-554.

132. Siebert, D.; Dahlem, J.; Fiechter, S.; Hartmann, A., An ESR Investigation of Synthetic Pyrite Crystals. Z. Naturforsch 1989, 44a, 59-66.

133. Mller, W.; Bertschat, H. H.; Biedermann, K.; Kowallik, R.; Lahmer-Naim, E.; Mahnke, H. E.; Seeger, S.; Zeitz, W. D.; Fiechter, S.; Tributsch, H., Perturbed- 
angular-distribution measurements of the chemical shift of iron in the disulfides FeS2 (pyrite) and RuS2 (laurite). Phys. Rev. B 1990, 41 (13), 8624-8629.

134. Birkholz, M.; Fiechter, S.; Hartmann, A.; Tributsch, H., Sulfur deficiency in iron pyrite (FeS2-x) and its consequences for band-structure models. Phys. Rev. B 1991, 43 (14), 926-936.

135. Pettenkofer, C.; Jaegermann, W.; Bronold, M., Site specific surface interaction of electron donors and acceptors on FeS2 (100) cleavage planes. Berichte der ... 1991, 2 (100), 560-565.

136. Siebert, D.; Stocker, W., Investigation of a (100) surface of pyrite by STM. Phys. status solidi 1992, 17, 17-20.

137. Ennaoui, A.; Schlichthorl, G.; Fiechter, S.; Tributsch, H., Vapor phase epitaxial growth of FeS2 pyrite and evaluation of the carrier collection in liquid-junction solar cell. Sol. Energy Mater. Sol. Cells 1992, 25 (1-2), 169-178.

138. Siebert, D.; Dahlem, J.; Fiechter, S.; Miller, R., EPR investigation of monovalent chromium centers in synthetic pyrite crystals. Phys. status solidi (b ... 1992, 93, 93-96.

139. Puff, W.; Birkholz, M.; Balogh, A. G.; Fiechter, S., Vacancy-Type Defects in IronPyrite FeS2-x. Mater. Sci. Forum 1997, 255-257, 342-344.

140. Diener, A.; Köppe, R., Synthesis of selenium doted pyrite single crystals prepared by chemical vapor transport. J. Cryst. Growth 2012, 349 (1), 55-60.

141. Zavašnik, J.; Rečnik, a., Electron microscopy study of CVT grown Fe-sulphides. J. Cryst. Growth 2013, 367, 18-23.

142. Nesbitt, H. W.; Muir, I. J., X-ray photoelectron spectroscopic study of a pristine pyrite surface reacted with water vapour and air. Geochim. Cosmochim. Acta 1994, 58 (21), 4667-4679.

143. Nesbitt, H. W.; Bancroft, G. M.; Pratt, A. R.; Scaini, M. J., Sulfur and iron surface states on fractured pyrite surfaces. Am. Mineral. 1998, 83 (9-10), 1067-1076.

144. Schaufuß, A. G.; Nesbitt, H. W.; Kartio, I.; Laajalehto, K.; Bancroft, G. M.; Szargan, R., Incipient oxidation of fractured pyrite surfaces in air. J. Electron Spectros. Relat. Phenomena 1998, 96 (1-3), 69-82.

145. Nesbitt, H. W.; Scaini, M.; Höchst, H.; Bancroft, G. M.; Schaufuss, a G.; Szargan, R., Synchrotron XPS evidence for Fe2+-S and Fe3+-S surface species on pyrite fracture-surfaces, and their 3D electronic states. Am. Mineral. 2000, 85 (1), 850857.

146. Uhlig, I.; Szargan, R.; Nesbitt, H. W.; Laajalehto, K., Surface states and reactivity of pyrite and marcasite. Appl. Surf. Sci. 2001, 179 (1-4), 222-229.

147. von Oertzen, G. U.; Skinner, W. M.; Nesbitt, H. W., Ab initio and X-ray photoemission spectroscopy study of the bulk and surface electronic structure of pyrite (100) with implications for reactivity. Phys. Rev. B 2005, 72 (23), 235427.

148. von Oertzen, G. U.; Skinner, W. M.; Nesbitt, H. W., Ab initio and XPS studies of pyrite (100) surface states. Radiat. Phys. Chem. 2006, 75 (11), 1855-1860.

149. van der Heide, P. A. W., Multiplet splitting patterns exhibited by the first row transition metal oxides in X-ray photoelectron spectroscopy. J. Electron Spectros. Relat. Phenomena 2008, 164 (1-3), 8-18. 
150. Schaufuß, A. G.; Nesbitt, H. W.; Kartio, I.; Laajalehto, K.; Bancroft, G. M.; Szargan, R., Reactivity of surface chemical states on fractured pyrite. Surf. Sci. 1998, 411 (3), 321-328.

151. Harmer, S. L.; Nesbitt, H. W., Stabilization of pyrite (FeS2), marcasite (FeS2), arsenopyrite (FeAsS) and loellingite (FeAs2) surfaces by polymerization and autoredox reactions. Surf. Sci. 2004, 564 (1-3), 38-52.

152. Berlich, A. G.; Nesbitt, H. W.; Bancroft, G. M.; Szargan, R., Binding of oxygen on vacuum fractured pyrite surfaces: Reactivity of iron and sulfur surface sites. Surf. Sci. 2013, 611, 60-66.

153. Doyle, C. S.; Kendelewicz, T.; Bostick, B. C.; Brown, G. E., Soft X-ray spectroscopic studies of the reaction of fractured pyrite surfaces with $\mathrm{Cr}(\mathrm{VI})$ containing aqueous solutions. Geochim. Cosmochim. Acta 2004, 68 (21), 42874299.

154. Nesbitt, H. W.; Berlich, A. G.; Harmer, S. L.; Uhlig, I.; Bancroft, G. M.; Szargan, R., Identification of pyrite valence band contributions usig synchrotron-excited Xray photoelectron spectroscopy. Am. Mineral. 2004, 89 (1), 382-389.

155. Eyert, V.; Höck, K. H.; Fiechter, S.; Tributsch, H., Electronic structure of FeS2: The crucial role of electron-lattice interaction. Phys. Rev. B 1998, 57 (11), 63506359. 


\section{Appendix: Calculated isotope abundances}

\section{For all combinations}

Abundances of different pyrite isotopes. The isotopes of each element are listed along with the molecular weight of the sum. The natural and modified amounts are calculated by multiplying the corresponding abundances of each isotope together. The variation is multiplying the abundances by two when the isotopes of sulfur are not the same to represent the two different forms $\mathrm{FeS}_{1} \mathrm{~S}_{2}$ and $\mathrm{FeS}_{2} \mathrm{~S}_{1}$. Without this the sum is not equal to1 as can be seen at the bottom of the table.

\begin{tabular}{|c|c|c|c|c|c|c|c|}
\hline $\mathrm{Fe}$ & S1 & S2 & MW & Natural & Variation $(2 \mathrm{x})$ & Modified & Variation $(2 \mathrm{x})$ \\
\hline 54 & 32 & 32 & 118 & $5.3 \mathrm{E}-02$ & $5.3 \mathrm{E}-02$ & $4.7 \mathrm{E}-02$ & $4.7 \mathrm{E}-02$ \\
\hline 54 & 32 & 33 & 119 & $4.2 \mathrm{E}-04$ & $8.4 \mathrm{E}-04$ & $3.8 \mathrm{E}-04$ & $7.6 \mathrm{E}-04$ \\
\hline 54 & 32 & 34 & 120 & $2.4 \mathrm{E}-03$ & $4.8 \mathrm{E}-03$ & $4.9 \mathrm{E}-03$ & $9.8 \mathrm{E}-03$ \\
\hline 54 & 32 & 36 & 122 & $1.1 \mathrm{E}-05$ & $2.2 \mathrm{E}-05$ & $1.0 \mathrm{E}-05$ & $2.0 \mathrm{E}-05$ \\
\hline 54 & 33 & 33 & 120 & $3.4 \mathrm{E}-06$ & $3.4 \mathrm{E}-06$ & $3.0 \mathrm{E}-06$ & $3.0 \mathrm{E}-06$ \\
\hline 54 & 33 & 34 & 121 & $1.9 \mathrm{E}-05$ & $3.8 \mathrm{E}-05$ & $3.9 \mathrm{E}-05$ & $7.8 \mathrm{E}-05$ \\
\hline 54 & 33 & 36 & 123 & $8.9 \mathrm{E}-08$ & $1.8 \mathrm{E}-07$ & $8.0 \mathrm{E}-08$ & $1.6 \mathrm{E}-07$ \\
\hline 54 & 34 & 34 & 122 & $1.1 \mathrm{E}-04$ & $1.1 \mathrm{E}-04$ & $5.1 \mathrm{E}-04$ & $5.1 \mathrm{E}-04$ \\
\hline 54 & 34 & 36 & 124 & $5.0 \mathrm{E}-07$ & $1.0 \mathrm{E}-06$ & $1.0 \mathrm{E}-06$ & $2.1 \mathrm{E}-06$ \\
\hline 54 & 36 & 36 & 126 & $2.3 \mathrm{E}-09$ & $2.3 \mathrm{E}-09$ & $2.1 \mathrm{E}-09$ & $2.1 \mathrm{E}-09$ \\
\hline 56 & 32 & 32 & 120 & $8.3 \mathrm{E}-01$ & $8.3 \mathrm{E}-01$ & $7.4 \mathrm{E}-01$ & $7.4 \mathrm{E}-01$ \\
\hline 56 & 32 & 33 & 121 & $6.6 \mathrm{E}-03$ & $1.3 \mathrm{E}-02$ & $5.9 \mathrm{E}-03$ & $1.2 \mathrm{E}-02$ \\
\hline 56 & 32 & 34 & 122 & $3.7 \mathrm{E}-02$ & $7.5 \mathrm{E}-02$ & $7.7 \mathrm{E}-02$ & $1.5 \mathrm{E}-01$ \\
\hline 56 & 32 & 36 & 124 & $1.7 \mathrm{E}-04$ & $3.5 \mathrm{E}-04$ & $1.6 \mathrm{E}-04$ & $3.1 \mathrm{E}-04$ \\
\hline 56 & 33 & 33 & 122 & $5.3 \mathrm{E}-05$ & $5.3 \mathrm{E}-05$ & $4.8 \mathrm{E}-05$ & $4.8 \mathrm{E}-05$ \\
\hline 56 & 33 & 34 & 123 & $3.0 \mathrm{E}-04$ & $6.0 \mathrm{E}-04$ & $6.2 \mathrm{E}-04$ & $1.2 \mathrm{E}-03$ \\
\hline 56 & 33 & 36 & 125 & $1.4 \mathrm{E}-06$ & $2.8 \mathrm{E}-06$ & $1.3 \mathrm{E}-06$ & $2.5 \mathrm{E}-06$ \\
\hline 56 & 34 & 34 & 124 & $1.7 \mathrm{E}-03$ & $1.7 \mathrm{E}-03$ & $8.0 \mathrm{E}-03$ & $8.0 \mathrm{E}-03$ \\
\hline 56 & 34 & 36 & 126 & $7.9 \mathrm{E}-06$ & $1.6 \mathrm{E}-05$ & $1.6 \mathrm{E}-05$ & $3.2 \mathrm{E}-05$ \\
\hline 56 & 36 & 36 & 128 & $3.7 \mathrm{E}-08$ & $3.7 \mathrm{E}-08$ & $3.3 \mathrm{E}-08$ & $3.3 \mathrm{E}-08$ \\
\hline 57 & 32 & 32 & 121 & $1.9 \mathrm{E}-02$ & $1.9 \mathrm{E}-02$ & $1.7 \mathrm{E}-02$ & $1.7 \mathrm{E}-02$ \\
\hline 57 & 32 & 33 & 122 & $1.5 \mathrm{E}-04$ & $3.1 \mathrm{E}-04$ & $1.4 \mathrm{E}-04$ & $2.7 \mathrm{E}-04$ \\
\hline 57 & 32 & 34 & 123 & $8.6 \mathrm{E}-04$ & $1.7 \mathrm{E}-03$ & $1.8 \mathrm{E}-03$ & $3.6 \mathrm{E}-03$ \\
\hline 57 & 32 & 36 & 125 & $4.0 \mathrm{E}-06$ & $8.0 \mathrm{E}-06$ & $3.6 \mathrm{E}-06$ & $7.2 \mathrm{E}-06$ \\
\hline 57 & 33 & 33 & 123 & $1.2 \mathrm{E}-06$ & $1.2 \mathrm{E}-06$ & $1.1 \mathrm{E}-06$ & $1.1 \mathrm{E}-06$ \\
\hline 57 & 33 & 34 & 124 & $6.9 \mathrm{E}-06$ & $1.4 \mathrm{E}-05$ & $1.4 \mathrm{E}-05$ & $2.8 \mathrm{E}-05$ \\
\hline 57 & 33 & 36 & 126 & $3.2 \mathrm{E}-08$ & $6.4 \mathrm{E}-08$ & $2.9 \mathrm{E}-08$ & $5.8 \mathrm{E}-08$ \\
\hline 57 & 34 & 34 & 125 & $3.9 \mathrm{E}-05$ & $3.9 \mathrm{E}-05$ & $1.8 \mathrm{E}-04$ & $1.8 \mathrm{E}-04$ \\
\hline 57 & 34 & 36 & 127 & $1.8 \mathrm{E}-07$ & $3.6 \mathrm{E}-07$ & $3.7 \mathrm{E}-07$ & $7.5 \mathrm{E}-07$ \\
\hline 57 & 36 & 36 & 129 & $8.5 \mathrm{E}-10$ & $8.5 \mathrm{E}-10$ & $7.6 \mathrm{E}-10$ & $7.6 \mathrm{E}-10$ \\
\hline
\end{tabular}




\begin{tabular}{|l|l|l|l|l|l|l|l|}
58 & 32 & 32 & 122 & $2.5 \mathrm{E}-03$ & $2.5 \mathrm{E}-03$ & $2.3 \mathrm{E}-03$ & $2.3 \mathrm{E}-03$ \\
\hline 58 & 32 & 33 & 123 & $2.0 \mathrm{E}-05$ & $4.1 \mathrm{E}-05$ & $1.8 \mathrm{E}-05$ & $3.7 \mathrm{E}-05$ \\
\hline 58 & 32 & 34 & 124 & $1.1 \mathrm{E}-04$ & $2.3 \mathrm{E}-04$ & $2.4 \mathrm{E}-04$ & $4.7 \mathrm{E}-04$ \\
\hline 58 & 32 & 36 & 126 & $5.4 \mathrm{E}-07$ & $1.1 \mathrm{E}-06$ & $4.8 \mathrm{E}-07$ & $9.6 \mathrm{E}-07$ \\
\hline 58 & 33 & 33 & 124 & $1.6 \mathrm{E}-07$ & $1.6 \mathrm{E}-07$ & $1.5 \mathrm{E}-07$ & $1.5 \mathrm{E}-07$ \\
\hline 58 & 33 & 34 & 125 & $9.2 \mathrm{E}-07$ & $1.8 \mathrm{E}-06$ & $1.9 \mathrm{E}-06$ & $3.8 \mathrm{E}-06$ \\
\hline 58 & 33 & 36 & 127 & $4.3 \mathrm{E}-09$ & $8.6 \mathrm{E}-09$ & $3.8 \mathrm{E}-09$ & $7.7 \mathrm{E}-09$ \\
\hline 58 & 34 & 34 & 126 & $5.2 \mathrm{E}-06$ & $5.2 \mathrm{E}-06$ & $2.5 \mathrm{E}-05$ & $2.5 \mathrm{E}-05$ \\
\hline 58 & 34 & 36 & 128 & $2.4 \mathrm{E}-08$ & $4.8 \mathrm{E}-08$ & $5.0 \mathrm{E}-08$ & $1.0 \mathrm{E}-07$ \\
\hline 58 & 36 & 36 & 130 & $1.1 \mathrm{E}-10$ & $1.1 \mathrm{E}-10$ & $1.0 \mathrm{E}-10$ & $1.0 \mathrm{E}-10$ \\
\hline
\end{tabular}

\section{For each molecular weight of pyrite}

Abundance of each molecular weight, each molecular weight's ratio of abundance to the abundance of the molecular weight of 120 for both the natural abundances and the modified abundances. The ratio of these two numbers to determine the biggest differences between the two.

\begin{tabular}{|c|c|c|c|c|c|}
\hline M.W. & FeS $2_{\mathrm{A}}$ & $\mathrm{x}_{\mathrm{A}} / 120_{\mathrm{A}}$ & $\mathrm{FeS}_{2 \mathrm{MA}}$ & $\mathrm{x}_{\mathrm{MA}} / 120_{\mathrm{MA}}$ & $\left(\mathrm{x}_{\mathrm{MA}} / 120_{\mathrm{MA}}\right) /\left(\mathrm{x}_{\mathrm{A}} / 120_{\mathrm{A}}\right)$ \\
\hline 118 & $5.3 \mathrm{E}-02$ & $6.3 \mathrm{E}-02$ & $4.7 \mathrm{E}-02$ & $6.3 \mathrm{E}-02$ & 1.0 \\
\hline 119 & $8.4 \mathrm{E}-04$ & $1.0 \mathrm{E}-03$ & $7.6 \mathrm{E}-04$ & $1.0 \mathrm{E}-03$ & 1.0 \\
\hline 120 & $8.3 \mathrm{E}-01$ & $1.0 \mathrm{E}+00$ & $7.5 \mathrm{E}-01$ & $1.0 \mathrm{E}+00$ & 1.0 \\
\hline 121 & $3.2 \mathrm{E}-02$ & $3.9 \mathrm{E}-02$ & $2.9 \mathrm{E}-02$ & $3.9 \mathrm{E}-02$ & 1.0 \\
\hline 122 & $7.8 \mathrm{E}-02$ & $9.4 \mathrm{E}-02$ & $1.6 \mathrm{E}-01$ & $2.1 \mathrm{E}-01$ & 2.2 \\
\hline 123 & $2.4 \mathrm{E}-03$ & $2.8 \mathrm{E}-03$ & $4.8 \mathrm{E}-03$ & $6.4 \mathrm{E}-03$ & 2.3 \\
\hline 124 & $2.3 \mathrm{E}-03$ & $2.7 \mathrm{E}-03$ & $8.8 \mathrm{E}-03$ & $1.2 \mathrm{E}-02$ & 4.3 \\
\hline 125 & $5.2 \mathrm{E}-05$ & $6.2 \mathrm{E}-05$ & $2.0 \mathrm{E}-04$ & $2.6 \mathrm{E}-04$ & 4.2 \\
\hline 126 & $2.2 \mathrm{E}-05$ & $2.7 \mathrm{E}-05$ & $5.8 \mathrm{E}-05$ & $7.7 \mathrm{E}-05$ & 2.9 \\
\hline 127 & $3.7 \mathrm{E}-07$ & $4.5 \mathrm{E}-07$ & $7.6 \mathrm{E}-07$ & $1.0 \mathrm{E}-06$ & 2.2 \\
\hline 128 & $8.5 \mathrm{E}-08$ & $1.0 \mathrm{E}-07$ & $1.3 \mathrm{E}-07$ & $1.8 \mathrm{E}-07$ & 1.7 \\
\hline 129 & $8.5 \mathrm{E}-10$ & $1.0 \mathrm{E}-09$ & $7.6 \mathrm{E}-10$ & $1.0 \mathrm{E}-09$ & 1.0 \\
\hline 130 & $1.1 \mathrm{E}-10$ & $1.4 \mathrm{E}-10$ & $1.0 \mathrm{E}-10$ & $1.3 \mathrm{E}-10$ & 1.0 \\
\hline
\end{tabular}

